\title{
Convective Linear Stability of Solitary Waves for Boussinesq Equations
}

\author{
By Robert L. Pego and Michael I. Weinstein
}

\begin{abstract}
Boussinesq was the first to explain the existence of Scott Russell's solitary wave mathematically. He employed a variety of asymptotically equivalent equations to describe water waves in the small-amplitude, long-wave regime. We study the linearized stability of solitary waves for three linearly well-posed Boussinesq models. These are problems for which well-developed Lyapunov methods of stability analysis appear to fail. However, we are able to analyze the eigenvalue problem for small-amplitude solitary waves, by comparison to the equation that Boussinesq himself used to describe the solitary wave, which is now called the Korteweg-de Vries equation. With respect to a weighted norm designed to diminish as perturbations convect away from the wave profile, we prove that nonzero eigenvalues are absent in a half-plane of the form $\Re \lambda>-b$ for some $b>0$, for all three Boussinesq models. This result is used to prove the decay of solutions of the evolution equations linearized about the solitary wave, in two of the models. This "convective linear stability" property has played a central role in the proof of nonlinear asymptotic stability of solitary-wave-like solutions in other systems.
\end{abstract}

Addresses for correspondence: Professor Robert L. Pego, Institute for Physical Science and Technology, University of Maryland, College Park, MD 20742, and Professor Michael I. Weinstein, Department of Mathematics, University of Michigan, Ann Arbor, MI 48109. 


\section{Boussinesq's equations}

In several works presented to the Paris Academy of Sciences in 1871 and 1872 , Boussinesq addressed the problem of the persistence of solitary waves of permanent form on a fluid interface [1-4]. This problem had been raised to prominence in the 1840 s by the observations of Scott Russell [5] and the objections of Airy [6] and Stokes [7].

Starting from Euler's equations for two-dimensional, inviscid, irrotational flow beneath a free surface, Boussinesq introduced approximations appropriate for long waves of small amplitude. To describe the nature of Boussinesq's approximations, we follow modern practice and nondimensionalize as follows. Let $a$ denote a typical surface wave amplitude, $h_{0}$, the uniform fluid depth when the fluid is at rest, and $l$, a characteristic length of a surface wave. Introduce the dimensionless ratios of lengths

$$
\alpha=a / h_{0}, \quad \beta=h_{0}^{2} / l^{2} \text {. }
$$

The Boussinesq scaling is defined by taking $\alpha=\beta=\varepsilon$ to be a small parameter, corresponding to studying long waves of small amplitude. A formal expansion may be carried out in powers of $\varepsilon$, neglecting terms of high order - see the book by Whitham [8]. The result of this procedure is a system of equations governing the time evolution of the nondimensional, scaled variables $\eta(x, t)$, the elevation of the free surface, and $u(x, t)$, the vertically averaged horizontal component of fluid velocity.

The most well known of Boussinesq's equations obtained in this way may be written

$$
\eta_{t t}=\eta_{x x}+\frac{3}{2} \varepsilon\left(\eta^{2}\right)_{x x}+\frac{1}{3} \varepsilon \eta_{x x x x}
$$

This equation is intended to describe waves moving basically in one direction, for which $\eta_{t}+\eta_{x}=O(\varepsilon)$. It gives a satisfactory description of steady long waves of small amplitude, has solitary-traveling-wave solutions, and admits an associated inverse scattering formalism, cf. [9, 10]. The solitary waves may be written explicitly, and they are unimodal and decay to a constant at $\pm \infty$. This Boussinesq equation has not been found suitable for the purpose of describing unsteady wave propagation, however, because its initial value problem is linearly ill posed. Linear plane waves $e^{i k x-i \omega t}$ obey the dispersion relation $\omega^{2}=k^{2}-\frac{1}{3} \varepsilon k^{4}$, which permits unbounded growth rates for high-frequency waves.

We concern ourselves with three other systems connected with Boussinesq's work for water waves, which have the advantage of being linearly well 
posed. These systems are treated in parallel in this work, and it will be convenient to refer to cases B1, B2, or B3, indicating to which system a particular equation corresponds.

The first equation may be obtained from that above by a modification that produces the same order of approximation. Since formally $\eta_{t t}=\eta_{x x}+O(\varepsilon)$, one obtains, to within terms that are $O\left(\varepsilon^{2}\right)$,

$$
\eta_{t t}=\eta_{x x}+\frac{3}{2} \varepsilon\left(\eta^{2}\right)_{x x}+\frac{1}{3} \varepsilon \eta_{x x t} .
$$

The linearization of this equation has a real dispersion relation, $\omega=$ $\pm k / \sqrt{1+\frac{1}{3} \varepsilon k^{2}}$, and the initial value problem has a rather simple local existence theory. Regarding global existence, it is known that some solutions develop singularities in finite time [11]. The sort of modification made in (1.1.B1) is a type of approximation made frequently by Boussinesq, although we have not found an equation exactly equivalent to (1.1.B1) in Boussinesq's works. The regularization in (1.1.B1) resembles that used by Benjamin et al. [12] to regularize the $\mathrm{KdV}$ equation, and (1.1.B1) has been derived by Rosenau [13] to model the (bidirectional) propagation of nonlinear waves in lattices.

The equations above are valid approximations for water waves only for waves propagating in one direction, but Boussinesq also derived related systems of equations that are appropriate for describing the bidirectional propagation of long waves of small amplitude. We are particularly interested in two systems that are described by Whitham [8], which have exactly the same linear dispersion relation as (1.1.B1). The first system ${ }^{1}$ is

$$
\begin{aligned}
\eta_{t}+u_{x}+\varepsilon(\eta u)_{x} & =0, \\
u_{t}+\eta_{x}+\varepsilon u u_{x}+\frac{1}{3} \varepsilon \eta_{t t x} & =0 .
\end{aligned}
$$

The initial value problem for (1.1.B2) has not been studied, to our knowledge. However, if one invokes the approximation $\eta_{t}+u_{x}=O(\varepsilon)$, one obtains a variant mentioned by Whitham [8, p. 466], which also appears in a paper of Peregrine [14]. This variant again has the same linear dispersion

\footnotetext{
${ }^{1}$ This system is nearly identical to one that received substantial attention by Boussinesq [15], consisting of his equations (270bis) and (276ter). The system (1.1.B2) differs only in that Boussinesq retained a nonlinear coefficient in front of the $\eta_{t t x}$ term. But Boussinesq does approximate this term as linear on page 391 , in passing.
} 
relation as (1.1.B1) and is given by the system ${ }^{2}$

$$
\begin{array}{r}
\eta_{t}+u_{x}+\varepsilon(\eta u)_{x}=0, \\
u_{t}+\eta_{x}+\varepsilon u u_{x}-\frac{1}{3} \varepsilon u_{t x x}=0 .
\end{array}
$$

The well posedness of the initial value problem for (1.1.B3) has been established, globally in time, by Schonbek [15] and Amick [16].

In deriving Equations (1.1) these days, one might prefer to proceed in reverse order. Whitham derives (1.1.B3), then relates it to (1.1.B2). Using the formal approximations $u_{t}+u_{x}=O(\varepsilon), \eta_{t}+\eta_{x}=O(\varepsilon)$, which are appropriate for unidirectional propagation, from (1.1.B3a) one finds $u=\eta+O(\varepsilon)$. Then (1.1.B1) can be derived, and from that, (1.1).

Each system of Boussinesq equations (1.1) has a two-parameter family of solitary-wave solutions that travel to the right-we describe these in Section 2. We are interested in stability properties of these waves, i.e., in the long-time behavior of solutions initially close to such a wave. Let us first discuss the physical heuristics that motivate our analysis. A small perturbation of a solitary traveling wave is expected to generate small-amplitude dispersive waves, and possibly small-amplitude solitary waves. We approximately describe small-amplitude dispersive waves as superpositions of plane waves $\exp (i k x-i \omega t)$, where $\omega=\omega(k)$ is a branch of the dispersion relation for the linearized equation. For the equations at hand, the group velocity of linear dispersive waves, $d \omega / d k$, is always strictly less than the speed of any solitary wave traveling to the right. Furthermore, small solitary waves travel slower than larger ones.

Thus, we expect that a slightly perturbed solitary wave will outrun the distortions generated by a small perturbation and recover its shape, after allowing for some adjustment of phase and amplitude. Equivalently, in a rightward-moving frame of reference in which the solitary wave is at rest, small disturbances should appear to convect away to the left, due to the mismatch between the solitary-traveling-wave speed and the admissible speeds of small disturbances. In this situation, we call the solitary traveling waves convectively stable.

How can we formulate the notion of convective stability mathematically? The heuristics above suggest that one introduce a weighted norm defined in terms of a frame of reference moving with the unperturbed solitary wave,

\footnotetext{
${ }^{2}$ The system (1.1.B3) has been associated with Boussinesq by several authors. As with (1.1.B1), we have not been able to find the system (1.1.B3) in Boussinesq's work. However, it is not unreasonable to associate these equations with Boussinesq. The approximations leading to (1.1.B1) and (1.1.B3) are entirely consistent with his method and style, which involved many fairly freely made approximations.
} 
designed to diminish as perturbations travel to the left. The solitary waves may be called convectively stable if they are asymptotically stable with respect to such a norm, modulo small adjustments of phase and amplitude.

To be more precise, suppose that the wave evolution equation can be written as a system $\varphi_{t}=\mathscr{F}(\varphi)$ and that a solitary wave traveling with speed $c$ is given by $\varphi_{c}(x-c t)$. We anticipate that if initial data $\varphi(x, 0)$ are close to $\varphi_{c}(x)$, then the solution approaches a solitary wave traveling with a nearby speed $c_{+}$with perhaps a small phase shift $\sigma_{+}$, in the sense that

$$
\begin{array}{r}
\varphi(x, t)=\varphi_{c_{+}}\left(x-c_{+} t-\sigma_{+}\right)+z\left(x-c_{+} t, t\right), \\
\text { where } z(s, t) \rightarrow 0 \text { as } t \rightarrow \infty
\end{array}
$$

in the weighted norm.

In our work on solitary wave stability for generalized KdV equations [17], we proved a convective stability result of just this type, finding that a convenient weighted norm is an exponentially weighted $L^{2}$ norm of the form

$$
\|f\|_{a}=\left\|e^{a s} f\right\|_{L^{2}}=\left(\int e^{2 a s}|f(s)|^{2} d s\right)^{1 / 2}
$$

where $a>0$ is sufficiently small. Note that for a function $f(s+C t)$ being convected to the left $(C>0)$, the norm $\|f(\cdot+C t)\|_{a} \rightarrow 0$ as $t \rightarrow \infty$ at an exponential rate.

An important part of the analysis in [17] is to establish what we call convective linear stability for the solitary waves. Suppose that in a frame of reference moving with the speed of the unperturbed solitary wave, the linearized evolution equation for the solitary wave perturbation, $z(s, t)$, is written in the form

$$
\partial_{t} z=\mathscr{A} z
$$

This equation has a two-parameter family of secular (nondecaying) solutions, corresponding to infinitesimal shifts in phase and changes in solitary wave speed. One wishes to show that Equation (1.4) is asymptotically stable with respect to the weighted norm in (1.3), modulo this two-parameter family.

The main purpose of this article is to prove this convective linear stability property for the small-amplitude solitary waves of the Boussinesq equations 
in (1.1), in cases B1 and B3. The main result is Theorem 11.1. In effect, we show that, with respect to a Hilbert space defined using the weighted norm in (1.3):

(i) The initial value problem for (1.4) is well posed, in that $\mathscr{A}$ is the generator of a $C_{0}$-semigroup (strongly continuous semigroup).

(ii) Zero is an isolated eigenvalue of $\mathscr{A}$ of multiplicity two.

(iii) Given initial data $z(s, 0)=z_{0}(s)$ for (1.4) whose spectral projection onto the generalized kernel of $\mathscr{A}$ vanishes, we have

$$
\|z(\cdot, t)\|_{a} \leq C e^{-b t}\left\|z_{0}\right\|_{a}, \quad t>0
$$

Something less is achieved in the case B2, due to the lack of a theory of well-posedness in that case. What is proved in all three cases (see Theorem 5.3) is the absence of nonzero eigenvalues satisfying $\mathfrak{R} \lambda>-b$ for some $b>0$, with respect to the weighted norm. This is the major part of proving convective linear stability in the cases B1 and B3.

In these cases, introducing the weighted norm has the effect of shifting the continuous spectrum of $\mathscr{A}$ from the imaginary axis into the left half-plane. Any nonzero eigenvalues that appear, which satisfy $\Re \lambda \leq 0$, correspond to what are called resonance poles ${ }^{3}$ for $\mathscr{A}$ with respect to the unweighted norm, and serve to limit the decay rate that may be obtained in (iii). In effect, we show that resonance poles are absent in a strip $-b<\mathfrak{R} \lambda \leq 0$ containing the imaginary axis.

To study the eigenvalue problem, we make use of the Evans function [23, 24], a Wronskian-like analytic function whose zeros in the right half-plane correspond to unstable eigenvalues. For the problems at hand, the natural domain of the Evans function extends some distance into the left half-plane. To locate its zeros, our strategy is to exploit the fact that in the small-amplitude, long-wave regime for unidirectional wave propagation, each of the Boussinesq equations (1.1) is asymptotically related to a fundamental and famous equation, the so-called Korteweg-de Vries equation:

$$
\eta_{t}+\eta_{x}+\frac{3}{2} \varepsilon \eta \eta_{x}+\frac{1}{6} \varepsilon \eta_{x x x}=0
$$

We prove that the Evans function for each eigenvalue problem from (1.1) converges (uniformly in a domain containing the closed right half-plane) to

\footnotetext{
${ }^{3}$ The term "resonance pole" is drawn from a phenomenon arising in quantum scattering theory [18]. The same phenomenon gives rise to the scattering frequencies in classical scattering theory [19] and "Landau damping" in plasma physics [20, 21, 22].
} 
that from the $\mathrm{KdV}$ equation, taken with an appropriate scaling. For the $\mathrm{KdV}$ equation, the Evans function is known explicitly, and it vanishes only at the origin. By means of the argument principle for analytic functions, we deduce, for small-amplitude solitary waves of each of the Boussinesq equations (1.1), that there are no nonzero eigenvalues satisfying $\Re \lambda>-b$, for some $b>0$.

Of course, it is insufficient to prove the absence of eigenvalues with $\Re \lambda>-b$, in order to prove a stability estimate such as (iii) for the $C_{0}$-semi$\operatorname{group} \exp (\mathscr{A} t)$, regarded as restricted to the spectral complement of the generalized kernel of $\mathscr{A}$. The spectrum of the operator $\mathscr{A}$ will not consist of eigenvalues only. And anyway, the location of the spectrum does not determine stability - examples are known in which the operator norm of a $C_{0}$-semigroup grows exponentially in time, while the spectrum of the generator lies in the left half of the complex plane.

Indeed, to infer the stability estimate (iii), we make use of a relatively recent result in semigroup theory, derived from Gearhart's strong spectral mapping theorem [25]. As is elegantly shown by Prüss [26] (also see [27]), given a $C_{0}$-semigroup $\exp (\mathscr{A} t)$ on a Hilbert space, a necessary and sufficient condition for uniform asymptotic stability is that the norm of the resolvent $(\lambda I-\mathscr{A})^{-1}$ be uniformly bounded for $\mathfrak{R} \lambda>0$. For the cases B1 and B3, we establish such uniform bounds in Section 11.

Convective stability, in the sense we have described, has recently been proved for solitary waves in a number of nonlinear dispersive systems. We have already referred to our results for generalized $\mathrm{KdV}$ equations [17]. Miller [28] and Miller and Weinstein [29] obtained similar results for solitary traveling waves of the regularized long-wave (RLW) equation. Dodd [30] has proved a convective stability result for shock profiles in a modified $\mathrm{KdV}-$ Burgers equation with cubic nonlinearity. In all these works, exponentially weighted Sobolev norms were used, similar to the norm in (1.3). For a class of nonlinear Schrödinger equations (NLS), such as arise in nonlinear optics and plasma physics, a closely related approach was introduced by Soffer and Weinstein [31-33] to prove the nonlinear asymptotic stability of solitary standing waves. Soffer and Weinstein used polynomially weighted $L^{2}$ norms and $L^{p}$ norms with $p>2$ to measure the transport and decay of dispersive waves in NLS.

In some dissipative systems, traveling waves that are unstable in a uniform norm may be convectively linearly stable and play a large role in the dynamics of solutions generally. One class of examples concerns model equations for gravity-driven waves on thin liquid films; see [34, 35] for reviews of work in this area. In certain parameter regimes, the uniform film is unstable to sufficiently long-wave perturbations, and solitary-traveling-wave solutions that are unstable for that reason exist. However, it is observed that 
typical solutions develop an irregular train of weakly interacting solitary pulses, with each pulse suppressing instabilities that grow slowly in the wake of the pulse preceding. Related problems are studied by Chang et al. [36] and Ogawa and Suzuki [37].

A further motivation for pursuing the present approach to stability for the Boussinesq equations (1.1), in particular, is the dramatic failure of a nowclassic method of proving nonlinear stability (modulo translations) for solitary waves. This classic method, introduced by Benjamin [38], is based on using a conserved functional associated with a Hamiltonian structure as a Lyapunov function for solutions constrained by other conserved functionals ("momenta"). At the heart of the success of the method is a variational characterization of the wave profile of interest as a constrained minimum of the Hamiltonian subject to fixed momenta. It has since been developed with considerable success to obtain orbital stability results in a wide variety of nonlinear systems; cf. [39, 40, 41, 42, 43-46] for example. The work of Cazenave and Lions [47] is closely related. Lyapunov-type stability arguments have been used in many other fluid problems; see [48, 49, 50]. For the Boussinesq systems under consideration, the Lyapunov method fails because, as a critical point of the appropriate constrained variational problem, the solitary wave is a saddle point of infinite index. The failure of this method for solitary waves in the case B1 for (1.1) and a related system is discussed by Smereka [51] and by Pego et al. [52]. The mode of failure is similar to that which occurs for the full Euler equations, as mentioned by Bona and Sachs [53].

\section{Historical remarks on the origin of the $\mathrm{KdV}$ equation}

It is a remarkable fact, and apparently little known, that Equation (1.5) appears explicitly in Boussinesq's massive 1877 Memoir, as Equation (283bis) in a footnote on page 360 (with a different, but recognizable, notation). Moreover, Boussinesq based his description of the solitary wave, and his account of its stability, on a pair of equations exactly equivalent to (1.5), written in his notation as

$$
\begin{gathered}
\frac{d h}{d t}+\frac{d \cdot h \omega}{d x}=0 \\
\frac{\omega}{\sqrt{g H}}=1+\frac{3 h}{4 H}+\frac{H^{2}}{6 h} \frac{d^{2} h}{d x^{2}} .
\end{gathered}
$$

In this notation, $H$ is constant and represents the depth of the fluid at infinity, $h$ represents the elevation of the wave, and $g$ is the gravitational constant. These are Equations (5a) and (7a) of [2], Equations (29) and (34) 
of [3], and Equations (283) and (291) of [4]. (single) Equation (40) of [3] and (292) of [4] is also exactly equivalent to (1.5).

The solitary waves obtained by Boussinesq [2-4] were exactly traveling wave solutions of his pair of equations (1.6)-(1.7), obtained by requiring that $\omega$ be constant in (1.6)-(1.7). It seems that in 1895 Korteweg and de Vries [54] were unaware of these works of Boussinesq. They refer only to the first Comptes Rendus note of Boussinesq [1], which sketches a different, less satisfactory treatment.

In addition to his discovery of (1.5), Boussinesq's rationale for the stability of the solitary waves of (1.5) has had a major influence on modern developments on the topic. Benjamin [38] credits Boussinesq for the idea that a certain conserved functional, which Boussinesq called the "moment of instability," is relevant for explaining the stability of solitary waves. This functional is now known as a Hamiltonian energy for (1.5). One hundred years after Boussinesq introduced this quantity, Benjamin [38] and Bona [55] used it as a Lyapunov functional to construct a rigorous proof of orbital stability for the solitary wave solutions of (1.5). Boussinesq's argument that the moment of instability is constant in time rests exactly on his version of Equation (1.5), namely Equations (1.6)-(1.7).

It is curious that, until now, no one seems to have noted the important role that (1.5) itself plays in Boussinesq's work, with one exception being Miles [56], who gives a rather thorough account. We refer the reader to that paper for further information. Miles did not press Boussinesq's priority for deriving (1.5), however, most likely because he appears not to have been aware of its explicit appearance in the 1877 Memoir.

\section{The KdV scaling and solitary waves}

We begin by developing the properties of the solitary waves of the Boussinesq equations (1.1) that are subsequently useful. It is convenient to rewrite the equations in a frame of reference moving with some (nondimensional) speed $c$, in which solitary waves appear as equilibria. In addition, we unscale (1.1) to obtain equations where the parameter $\varepsilon$ does not appear. To do this, define

$$
\eta=\frac{1}{3 \varepsilon} \tilde{\eta}, \quad u=\frac{c}{3 \varepsilon} \tilde{u}, \quad \tau=c \sqrt{\frac{3}{\varepsilon}} t, \quad s=\sqrt{\frac{3}{\varepsilon}}(x-c t)
$$

Carrying out this change of variables in (1.1) for each case gives respectively

$$
\left(I-\partial_{s}^{2}\right)\left(\partial_{\tau}-\partial_{s}\right)^{2} \tilde{\eta}-\partial_{s}^{2}\left(c^{-2} \tilde{\eta}+\frac{1}{2} \tilde{\eta}^{2}\right)=0
$$




$$
\begin{array}{r}
\left(\partial_{\tau}-\partial_{s}\right) \tilde{\eta}+\partial_{s}\left(\tilde{u}+\frac{1}{3} \tilde{\eta} \tilde{u}\right)=0 \\
\left(\partial_{\tau}-\partial_{s}\right) \tilde{u}+\partial_{s}\left(c^{-2} \tilde{\eta}+\frac{1}{6} \tilde{u}^{2}+\left(\partial_{\tau}-\partial_{s}\right)^{2} \tilde{\eta}\right)=0 \\
\left(\partial_{\tau}-\partial_{s}\right) \tilde{\eta}+\partial_{s}\left(\tilde{u}+\frac{1}{3} \tilde{\eta} \tilde{u}\right)=0 \\
\left(I-\partial_{s}^{2}\right)\left(\partial_{\tau}-\partial_{s}\right) \tilde{u}+\partial_{s}\left(c^{-2} \tilde{\eta}+\frac{1}{6} \tilde{u}^{2}\right)=0 .
\end{array}
$$

(In (2.1.B1) we have used the definition $\eta=c^{2} \tilde{\eta} / 3 \varepsilon$ instead.)

We study the stability properties of solitary traveling waves in the so-called Korteweg-de Vries (KdV) limit. We introduce the parameter $\gamma$ by defining

$$
c^{-2}=1-\gamma^{2}
$$

The parameter $c$ corresponds to the Froude number in the exact theory of water waves. We presume that $c>0$, without loss of generality. The $K d V$ limit corresponds to taking $c^{2} \rightarrow 1$, or $\gamma \rightarrow 0$. In this process, $\varepsilon$ is regarded as fixed.

The $K d V$ scaling is defined by

$$
\tilde{\eta}=\gamma^{2} \eta_{*}(\xi, T), \quad \tilde{u}=\gamma^{2} u_{*}(\xi, T), \quad \text { where } \xi=\gamma s, T=\frac{1}{2} \gamma^{3} \tau
$$

In the $\mathrm{KdV}$ limit, for the Boussinesq equations in (2.1), one finds that formally $u_{*}=\eta_{*}+O\left(\gamma^{2}\right)$ and that up to terms formally of order $O\left(\gamma^{2}\right), \eta_{*}$ satisfies the Korteweg-de Vries equation in the form

$$
\partial_{T} \eta_{*}-\partial_{\xi} \eta_{*}+\partial_{\xi}\left(\frac{1}{2} \eta_{*}^{2}\right)+\partial_{\dot{\xi}}^{3} \eta_{*}=0
$$

Solitary waves of (1.1) moving with speed $c$ correspond to time-independent solutions of (2.1) that vanish in the limit $s \rightarrow \pm \infty$. Seeking solutions $\tilde{\eta}=\tilde{\eta}_{c}(s), \tilde{u}=\tilde{u}_{c}(s)$ (where subscripts no longer denote derivatives), we require respectively

$$
\begin{gathered}
\tilde{\eta}_{c}^{\prime \prime}-\gamma^{2} \tilde{\eta}_{c}+\frac{1}{2} \tilde{\eta}_{c}^{2}=0, \\
\tilde{\eta}_{c}^{\prime \prime}-\gamma^{2} \tilde{\eta}_{c}+\frac{1}{2} \tilde{\eta}_{c}^{2} \frac{9+2 \tilde{\eta}_{c}}{\left(3+\tilde{\eta}_{c}\right)^{2}}=0, \quad \tilde{u}_{c}=\frac{\tilde{\eta}_{c}}{1+\frac{1}{3} \tilde{\eta}_{c}}, \\
\tilde{u}_{c}^{\prime \prime}-\gamma^{2} \tilde{u}_{c}+\frac{1}{6} \tilde{u}_{c}^{2}+\frac{\left(1-\gamma^{2}\right) \tilde{u}_{c}^{2}}{3-\tilde{u}_{c}}=0, \quad \tilde{\eta}_{c}=\frac{\tilde{u}_{c}}{1-\frac{1}{3} \tilde{u}_{c}}
\end{gathered}
$$

The solitary wave for the case B1 is given explicitly by $\tilde{\eta}_{c}(s)=3 \gamma^{2} \operatorname{sech}^{2}\left(\frac{1}{2} \gamma s\right)$. 
Introduce the $\mathrm{KdV}$ scaling

$$
\tilde{\eta}_{c}=\gamma^{2} \eta_{*}(\xi, \gamma), \quad \tilde{u}_{c}=\gamma^{2} u_{*}(\xi, \gamma)
$$

In the KdV limit $\gamma \rightarrow 0$, we find that $u_{*}=\eta_{*}+\mathscr{O}\left(\gamma^{2}\right)$ for the cases B2 and $\mathrm{B} 3$, and in all cases it turns out that

$$
\eta_{*}^{\prime \prime}-\eta_{*}+\frac{1}{2} \eta_{*}^{2}=\mathscr{O}\left(\gamma^{2} \eta_{*}^{2}\right)
$$

The solitary wave $\eta_{*}=\Theta(\xi)$ of the $\mathrm{KdV}$ equation (2.4) satisfies

$$
\Theta^{\prime \prime}-\Theta+\frac{1}{2} \Theta^{2}=0
$$

It is given explicitly by

$$
\Theta(\xi)=3 \operatorname{sech}^{2}\left(\frac{1}{2} \xi\right) .
$$

Note that for the case B1 we have $\eta_{*}=\Theta$ exactly. For the other two cases, the properties of solitary wave solutions are summarized by the following result.

THEOREM 2.1. In the cases B2 and B3, for any nonzero $\gamma \in[0,1)$, Equation (2.5) has a unique solution $\left(\tilde{\eta}_{c}, \tilde{u}_{c}\right)$ with positive, even components that approach 0 as $s \rightarrow \infty$. In the KdV scaling (2.6), the functions $\eta_{*}$ and $u_{*}$ are real analytic in their arguments $(\xi, \gamma) \in \mathbb{R} \times[0,1)$. When $\gamma=0$ we have $\eta_{*}=u_{*}=\Theta$. Furthermore, for $\gamma$ in any compact subset of $[0,1)$,

$$
\partial_{\dot{\xi}}^{j} \eta_{*}(\xi, \gamma) e^{ \pm \xi}(\operatorname{sgn} \xi)^{j} \rightarrow K_{j}(\gamma) \quad \text { as } \xi \rightarrow \pm \infty, \text { for } j=0,1,2
$$

uniformly in $\gamma$, where $K_{j}$ is a nonvanishing continuous function of $\gamma$, for $j=0,1,2$.

Proof: First consider case B2. Let $v=\tilde{\eta}_{c}$ and suppose $\gamma \neq 0$. Then we require

$$
v^{\prime \prime}-\gamma^{2} v+F^{\prime}(v)=0
$$

where $F(v)=\frac{1}{2} v^{3} /(3+v)$. In the phase plane of this equation, the origin is a saddle point. Equation (2.10) has the first integral $E=\frac{1}{2} v^{\prime 2}-\frac{1}{2} \gamma^{2} v^{2}+$ 
$F(v)$. There is a unique $v_{m}=v_{m}(\gamma)>0$ such that $F\left(v_{m}\right)-\frac{1}{2} \gamma^{2} v_{m}^{2}=0$, given explicitly by $v_{m}=3 \gamma^{2} /\left(1-\gamma^{2}\right)$. The solution of (2.10) having $v(0)=v_{m}$, $v^{\prime}(0)=0$ has $E=0$ and it follows that this solution is even, positive, and approaches zero as $s \rightarrow \pm \infty$. In the $\mathrm{KdV}$ scaling $v(s)=\gamma^{2} v_{*}(\xi, \gamma)$, it is easy to see that $v_{*}$ is real analytic in $\xi$ and $\gamma$ for $(\xi, \gamma) \in \mathbb{R} \times[0,1)$, and $v_{*}(\xi, 0)=\Theta(\xi)$.

That the limits $K_{j}(\gamma)=\lim _{\xi \rightarrow \pm \infty} e^{ \pm \xi} \partial_{\xi}^{j} v_{*}(\xi, \gamma)$ exist is a standard conclusion of the stable manifold theorem. By following a standard proof of this theorem (cf. [57] for example), it is straightforward to verify that the limit is attained uniformly on compact sets, so $K_{j}$ is continuous.

Finally, consider the case B3. We proceed in a similar way as above, this time with $v=\tilde{u}_{c}$. The function $F$ in (2.10) is now determined from $F^{\prime}(v)=$ $v^{2}\left(\frac{1}{6}+\left(1-\gamma^{2}\right) /(3-v)\right)$. We have $F(0)=F^{\prime}(0)=F^{\prime \prime}(0)=0, F^{\prime}$ is an increasing convex function, and $F(v)$ increases from 0 to $\infty$ as $v$ goes from 0 to 3. It follows that a unique $v_{m}$ exists as above, depending analytically on $\gamma$, with $0<v_{m}<3$. The remainder of the proof goes the same as above.

Corollary 2.2. Given a compact interval $\left[0, \gamma_{0}\right] \subset[0,1)$, there exists a constant $K_{*}$ such that for $\gamma \in\left[0, \gamma_{0}\right]$, we have

$$
\left|\partial_{\dot{\xi}}^{j} \eta_{*}(\xi)\right| \leq K_{*} e^{-|\xi|} \quad \text { for } j=0,1,2, \quad-\infty<\xi<\infty,
$$

or equivalently

$$
\left|\partial_{s}^{j} \tilde{\eta}_{c}(s)\right| \leq K_{*} \gamma^{2+j} e^{-|\gamma s|} \quad \text { for } j=0,1,2, \quad-\infty<s<\infty .
$$

\section{The eigenvalue problem}

In this section, we display the eigenvalue problems associated with the solitary waves of the Boussinesq equations in (2.1), and we observe, at a formal level, how the eigenvalue problem for the KdV solitary wave emerges in the KdV limit.

For each of the Boussinesq equations in (2.1), we substitute

$$
\tilde{\eta}(s, \tau)=\tilde{\eta}_{c}(s)+h(s, \tau), \quad \tilde{u}(s, \tau)=\tilde{u}_{c}(s)+v(s, \tau),
$$

and obtain linearized evolution equations for $h(s, \tau)$ and $v(s, \tau)$ by retaining only terms that are linear in $h$ and $v$. We then seek solutions of the linearized evolution equations of the form $h(s) e^{\lambda \tau}$ and $v(s) e^{\lambda \tau}$. Corresponding to each equation in (2.1) respectively, we obtain the following eigenvalue equations:

$$
\left(I-\partial_{s}^{2}\right)\left(\lambda-\partial_{s}\right)^{2} h-\partial_{s}^{2}\left(c^{-2}+\tilde{\eta}_{c}\right) h=0,
$$




$$
\begin{gathered}
\left(\lambda-\partial_{s}\right) h+\partial_{s} v+\frac{1}{3} \partial_{s}\left(\tilde{u}_{c} h+\tilde{\eta}_{c} v\right)=0, \\
\left(\lambda-\partial_{s}\right) v+\left(c^{-2}+\left(\lambda-\partial_{s}\right)^{2}\right) \partial_{s} h+\frac{1}{3} \partial_{s}\left(\tilde{u}_{c} v\right)=0, \\
\left(\lambda-\partial_{s}\right) h+\partial_{s} v+\frac{1}{3} \partial_{s}\left(\tilde{u}_{c} h+\tilde{\eta}_{c} v\right)=0, \\
\left(I-\partial_{s}^{2}\right)\left(\lambda-\partial_{s}\right) v+c^{-2} \partial_{s} h+\frac{1}{3} \partial_{s}\left(\tilde{u}_{c} v\right)=0 .
\end{gathered}
$$

The corresponding linearized evolution equations for $h(s, \tau)$ and $v(s, \tau)$ may be written down by replacing $\lambda$ by $\partial_{\tau}$ in (3.1). Each eigenvalue problem in (3.1) admits a symmetry usually associated with Hamiltonian systems. One may replace $\lambda$ by $-\lambda$ and reflect in space, replacing $s$ by $-s$. It follows that if $\lambda$ is an eigenvalue (with respect to an unweighted or symmetrically weighted norm), then $-\lambda$ is also.

Now we introduce a $\mathrm{KdV}$ scaling of these eigenvalue problems. Recalling the scaling in (2.4), let

$$
\lambda=\frac{1}{2} \gamma^{3} \Lambda, \quad h(s)=\gamma^{2} H(\xi), \quad v(s)=\gamma^{2} V(\xi), \quad \text { where } \xi=\gamma s .
$$

The eigenvalue problems in the KdV scaling are given by

$$
\begin{gathered}
\partial_{\xi}\left[\Lambda H+\partial_{\xi}^{3} H-\partial_{\xi} H+\partial_{\xi}(\Theta H)\right]=\gamma^{2}\left[\Lambda \partial_{\xi}^{3}+\frac{1}{4} \Lambda^{2}\left(I-\gamma^{2} \partial_{\xi}^{2}\right)\right] H, \\
\partial_{\xi}(H-V)=\gamma^{2}\left[\frac{1}{2} \Lambda H+\frac{1}{3} \partial_{\xi}\left(u_{*} H+\eta_{*} V\right)\right] \\
\frac{1}{2} \Lambda(H+V)+\partial_{\xi}^{3} H-\partial_{\xi} H+\frac{1}{3} \partial_{\xi}\left(u_{*} H+\left(u_{*}+\eta_{*}\right) V\right) \\
=\gamma^{2} \Lambda \partial_{\xi}^{2} H+\frac{1}{4} \gamma^{4} \Lambda^{2} \partial_{\xi} H \\
\partial_{\xi}(H-V)=\gamma^{2}\left[\frac{1}{2} \Lambda H+\frac{1}{3} \partial_{\xi}\left(u_{*} H+\eta_{*} V\right)\right] \\
\frac{1}{2} \Lambda(H+V)+\partial_{\xi}^{3} V-\partial_{\xi} H+\frac{1}{3} \partial_{\xi}\left(u_{*} H+\left(u_{*}+\eta_{*}\right) V\right) \\
=\frac{1}{2} \gamma^{2} \Lambda \partial_{\xi}^{2} V .
\end{gathered}
$$

The second equation in (3.3.B2) (resp. in (3.3.B3)) is obtained by adding the equations in (3.1.B2) (resp. in (3.1.B3)), then scaling. This is done to make more clear how the $\mathrm{KdV}$ eigenvalue problem emerges in the limit $\gamma \rightarrow 0$. Indeed, in this limit it is simple to observe that each of the eigenvalue problems in (3.3) formally reduces to the equation

$$
\Lambda H+\partial_{\xi}^{3} H-\partial_{\xi} H+\partial_{\xi}(\Theta H)=0 .
$$

This is the eigenvalue problem that arises from linearization of the $\mathrm{KdV}$ equation (2.4) about its solitary wave, $\Theta(\xi)$, the solution of (2.8). It is known from previous work $[17,58,59,60]$ that if $\Lambda \neq 0$ and $\Re \lambda \geq 0$, then (3.4) has 
no nontrivial solution $H$ in $L^{2}$. Regarding the eigenvalue problems in (3.1), our objective is to use what is known about the $\mathrm{KdV}$ eigenvalue problem to deduce that for sufficiently small $\gamma>0$, and some $a>0$, the eigenvalue problem in (3.1) has no solution with $e^{a s} h$ and $e^{a s} v$ in $L^{2}$, for any nonzero $\lambda$ in a half-plane of the form $\mathfrak{R} \lambda>-b, b>0$.

\section{The Evans function}

To analyze each eigenvalue problem displayed in Section 3, we define an Evans function $D(\lambda, \gamma)$, an analytic function designed so its zeros correspond to eigenvalues when $\mathfrak{R} \lambda>0$. This function was introduced by J. W. Evans [24] to study traveling wave stability in reaction-diffusion systems modeling nerve impulses; further applications were given by Jones [61] and Alexander et al. [23]. A systematic development of the basic properties of the Evans function was given by Pego and Weinstein [60], whose analysis was applied in particular to the single equation (3.1.B1), regarding $c^{2}>1$ as fixed. Here we are interested in studying $D(\lambda, \gamma)$ as a function of both $\lambda$ and $\gamma$, in the singular limit $\gamma \rightarrow 0$ corresponding to the limit $c^{2} \rightarrow 1^{+}$. We begin with a general description, then proceed to detail the results we need in Section 5 for stating the main results of this article.

To describe the Evans function as it may be defined for the eigenvalue problems in Section 3, we begin with the observation that each of these eigenvalue problems can be written, by a standard reduction, as a first-order homogeneous system of ordinary differential equations, e.g.,

$$
\frac{d y}{d s}=A(s, \lambda, \gamma) y
$$

Since the solitary waves decay exponentially as $s \rightarrow \pm \infty$, the coefficient matrix in Equation (4.1) converges rapidly, as $s \rightarrow \pm \infty$, to that of a constant coefficient system

$$
\frac{d y}{d s}=A^{\infty}(\lambda, \gamma) y
$$

Equation (4.2) has the solution $y=e^{\mu s} \mathbf{v}$ whenever $\mu$ is an eigenvalue of the matrix $A^{\infty}(\lambda, \gamma)$ and $\mathbf{v}$ is a corresponding eigenvector. Our systems have the feature that when $\mathfrak{R} \lambda \geq 0$, the matrix $A^{\infty}(\lambda, \gamma)$ has a unique eigenvalue with negative real part. Thus the eigenvalues of $A^{\infty}(\lambda, \gamma)$ may be labeled so that for $\mathfrak{R} \lambda \geq 0$,

$$
\mathfrak{R} \mu_{1}(\lambda, \gamma)<0 \leq \min _{j>1} \mathfrak{R} \mu_{j}(\lambda, \gamma)
$$


Corresponding to the solution $e^{\mu_{1} s} \mathbf{v}_{1}$ of (4.2), Equation (4.1) has a unique solution that decays to zero as $s \rightarrow \infty$, denoted $y^{+}(s, \lambda, \gamma)$, with a normalization chosen so that

$$
y^{+}(s, \lambda, \gamma) \sim e^{\mu_{1} s} \mathbf{v}_{1} \quad \text { as s } \rightarrow+\infty .
$$

For $\Re \lambda>0$, then, any solution of (4.1) that decays to zero as $s \rightarrow \infty$ must be a constant multiple of $y^{+}$. The Evans function $D(\lambda, \gamma)$ may be characterized by the property

$$
y^{+}(s, \lambda, \gamma) \sim D(\lambda, \gamma) e^{\mu_{1} s} \mathbf{v}_{1} \quad \text { as } s \rightarrow-\infty .
$$

That is, the Evans function serves as a transmission coefficient for $y^{+}$. It turns out that, for $\mathfrak{i} \lambda>0, y^{+}(s, \lambda, \gamma) \rightarrow 0$ as $s \rightarrow-\infty$ if and only if $D(\lambda, \gamma)=0$. From this, we find that, for $\Re \lambda>0, \lambda$ is an eigenvalue with respect to an unweighted norm if and only if $D(\lambda, \gamma)=0$.

In this article, the Evans function is defined in a vertical strip containing the imaginary axis, in addition to the right half-plane. According to the theory developed in [60], for $\gamma$ fixed, an Evans function may be defined naturally for (4.1) for all $\lambda$ belonging to a simply connected domain $\Omega^{\gamma}$ in the complex plane, provided that the following four hypotheses hold:

$\mathrm{H} 1 A(s, \lambda, \gamma)$ is continuous in $(s, \lambda)$ and is analytic in $\lambda$ for fixed $s$.

$\mathrm{H} 2 A(s, \lambda, \gamma) \rightarrow A^{\infty}(\lambda, \gamma)$ as $s \rightarrow \pm \infty$, uniformly for $\lambda$ in any compact subset of $\Omega^{\gamma}$.

H3 The integral $\int_{-\infty}^{\infty}\left|A(s, \lambda, \gamma)-A^{\infty}(\lambda, \gamma)\right| d s$ converges for all $\lambda \in \Omega^{\gamma}$, uniformly on compact subsets.

H4 For every $\lambda \in \Omega^{\gamma}, A^{\infty}(\lambda, \gamma)$ has a unique eigenvalue of smallest real part, which is simple.

For the eigenvalue problems in (3.1), the structural hypotheses $\mathrm{H} 1, \mathrm{H} 2$, and $\mathrm{H} 3$ are straightforward to verify - see Section 7, where we display explicitly the matrices $A(s, \lambda, \gamma)$ and $A^{\infty}(\lambda, \gamma)$ corresponding to each case. Compared with (4.3), hypothesis $\mathrm{H} 4$ simply requires that the eigenvalues of $A^{\infty}(\lambda, \gamma)$ can always be labeled so that

$$
\Re \mu_{1}(\lambda, \gamma)<\mu_{*}(\lambda, \gamma) \equiv \min _{j>1} \Re \mu_{j}(\lambda, \gamma) .
$$

It takes some trouble to describe a suitable domain $\Omega^{\gamma}$ on which (4.6) holds, because of a singular property of the KdV limit-as it turns out, $A^{\infty}$ has the quadruple eigenvalue $\mu=0$ when $(\lambda, \gamma)=(0,0)$.

If we suppose that we have a domain $\Omega^{\gamma}$ on which hypotheses $\mathrm{H} 1-\mathrm{H} 4$ hold, then for $\lambda \in \Omega^{\gamma}$ the Evans function $D(\lambda, \gamma)$ is characterized by (4.4) 
and (4.5), exactly as above. In general, what happens when the Evans function vanishes is described by the following result, drawn from [60].

Proposition 4.1. Suppose H1-H4 hold on $\Omega^{\gamma}$. Then for $\lambda \in \Omega^{\gamma}$, the following are equivalent:

(i) $D(\lambda, \gamma)=0$.

(ii) $y^{+}(s, \lambda, \gamma)=o\left(e^{\mu_{1} s}\right)$ as $s \rightarrow-\infty$.

(iii) $y^{+}(s, \lambda, \gamma)=O\left(e^{\mu * s}|s|^{r-1}\right)$ as $s \rightarrow-\infty$.

(In part (iii) of this proposition, $r=r(\lambda, \gamma)$ denotes the maximum algebraic multiplicity of any eigenvalue $\mu_{j}$ of $A^{\infty}(\lambda, \gamma)$. Generally the eigenvalues are distinct and simple so $r=1$. When $r>1$, however, one must strengthen assumption $\mathrm{H} 3$ slightly by multiplying the integrand by $|s|^{r-1}$.)

For the eigenvalue problems in Section 3, it turns out that $\mu_{*}(\lambda, \gamma)$ has the same sign as $\Re \lambda$. This is why $y^{+}(s, \lambda, \gamma) \rightarrow 0$ as $s \rightarrow-\infty$ if and only if $D(\lambda, \gamma)=0$, for $\mathfrak{i} \lambda>0$. It will follow that if $\mathfrak{R} \lambda>0, \lambda$ is an eigenvalue with respect to the unweighted $L^{2}$ norm if and only if $D(\lambda, \gamma)=0$.

With respect to the weighted norm in (1.3), we show that for $\lambda$ in a half-plane of the form $\Re \lambda>-b, b>0, \lambda$ is an eigenvalue if and only if $e^{a s} y^{+}(s, \lambda, \gamma) \rightarrow 0$ as $s \rightarrow \pm \infty$. By Proposition 4.1, this is true if and only if $D(\lambda, \gamma)=0$, provided $(\lambda, \gamma)$ is such that

$$
\mathfrak{R} \mu_{1}+a<0<\mathfrak{R} \mu_{j}+a \quad \text { for } j>1 \text {. }
$$

Below, we describe suitable conditions under which this relationship holds, whenever $\mathfrak{R} \lambda>-b$.

In the remainder of this section, we study the Evans functions specifically associated with the eigenvalue problems in (3.1) and (3.3) respectively, with particular emphasis on the domain. Also we describe the properties of the Evans function, for the eigenvalue problem (3.4) arising from the $\mathrm{KdV}$ equation, which have been proved previously in $[17,60]$.

\subsection{The Evans function in unscaled variables}

Consider the eigenvalue problems in (3.1). For each system in (3.1) respectively, the reduction to the form (4.1) is defined by setting

$$
y^{t}=\left(h, \partial_{s} h, \partial_{s}^{2} h, \partial_{s}^{3} h\right),\left(h, \partial_{s} h, \partial_{s}^{2} h, v\right), \text { or }\left(v, \partial_{s} v, \partial_{s}^{2} v, h\right)
$$

(Here $y^{t}$ denotes the transpose of the vector $y$.) The characteristic polynomial of the corresponding matrix $A^{\infty}(\lambda, \gamma)$ is determined by the linear dispersion relation of the equations in (2.1). The latter are all precisely the 
same, and we find that in each case, the characteristic polynomial of $A^{\infty}(\lambda, \gamma)$ is given by

$$
\mathscr{P}(\mu)=\mathscr{P}(\mu ; \lambda, \gamma)=\left(\mu^{2}-1\right)(\lambda-\mu)^{2}+\left(1-\gamma^{2}\right) \mu^{2}
$$

Some basic facts about the eigenvalues $\mu_{j}$ of $A^{\infty}(\lambda, \gamma)$ are summarized as follows. The proof is in Section 6.

LEMMA 4.2. (a) Suppose $0<\gamma<1$. Then for an appropriate labeling of the zeros $\mu_{j}$ of $\mathscr{P}(\mu)$, we have:

(i) $\mathfrak{R} \mu_{1}<0<\Re \mu_{j}$ for $j=2,3,4$, when $\mathfrak{R} \lambda>0$.

(ii) $\mathfrak{R} \mu_{1}<0=\mathfrak{R} \mu_{2}=\mathfrak{R} \mu_{3}<\mathfrak{R} \mu_{4}$, when $\mathfrak{R} \lambda=0$.

(iii) $\mathfrak{R} \mu_{j}<0<\Re \mu_{4}$ for $j=1,2,3$, when $\mathfrak{R} \lambda<0$.

(b) If $a=c_{0} \gamma$ where $0<c_{0}<1 / \sqrt{3}$, and if $\gamma>0$ is sufficiently small, then there exists $b>0$, such that if $\mathfrak{R} \lambda>-b$ then

$$
\mathfrak{R} \mu_{1}<-a<\Re \mu_{j} \quad \text { for } j=2,3,4 .
$$

It turns out that the two eigenvalues of smallest real part meet when

$$
\lambda=-\lambda_{*}(\gamma)=-\frac{1}{2} \gamma^{3} \Lambda_{*}(\gamma), \quad \Lambda_{*}(\gamma)=2 \gamma^{-3}\left(1-\left(1-\gamma^{2}\right)^{1 / 3}\right)^{3 / 2}
$$

(Note that $\Lambda_{*}$ is continuous for $\gamma \in[0,1)$ if we define $\Lambda_{*}(0)=\sqrt{\frac{4}{27}}$.) This collision of eigenvalues largely explains why the domain $\Omega^{\gamma}$ is defined as it is in the following proposition, with a cut along the negative real axis.

Proposition 4.3. Put $\varepsilon_{0}=0.7$. There exists $\gamma_{0} \in(0,1)$, such that if $0 \leq \gamma \leq$ $\gamma_{0}$, then hypothesis $H 4$ holds with $\Omega^{\gamma}$ defined by

$$
\Omega^{\gamma}=\left\{\lambda \mid \Re \lambda \geq-\varepsilon_{0}\right\} \backslash\left(-\infty,-\lambda_{*}(\gamma)\right]
$$

That is, for $\lambda \in \Omega^{\gamma}$ the matrix $A^{\infty}(\lambda, \gamma)$ has a unique eigenvalue of smallest real part, which is simple.

This proposition is proved in Section 6. Note that $0 \notin \Omega^{0}$ ! The numbers $\gamma_{0}$ and $\varepsilon_{0}$ described in Proposition 4.3 may be regarded as fixed in the remainder of this article apart from Section 6.

Now the domain and regularity of the Evans function may be described as follows. The proof is completed in Sections 7 and 8.

Proposition 4.4. For the system (4.1) corresponding to each system in (3.1), the Evans function $D(\lambda, \gamma)$ is analytic in $\lambda$, and jointly continuous in $(\lambda, \gamma)$, in 
the set $\Omega$ defined by

$$
\Omega=\left\{(\lambda, \gamma) \mid 0 \leq \gamma \leq \gamma_{0} \text { and } \lambda \in \Omega^{\gamma}\right\}
$$

Proposition 4.5. If $a=c_{0} \gamma$, where $0<c_{0}<1 / \sqrt{3}$ and $\gamma>0$ is sufficiently small, then there exists $b>0$, such that if $\Re \lambda>-b$, then each eigenvalue problem in (3.1) has a nontrivial solution such that $\|h\|_{a}$ and $\|v\|_{a}$ are finite, if and only if $D(\lambda, \gamma)=0$.

Proof: By Lemma 4.2(b), if $\gamma>0$ is sufficiently small then there exists $b>0$ as stated, such that whenever $\mathfrak{R} \lambda>-b$, the conclusions of Lemma 4.2(b) hold. By Proposition 4.1, if $\mathfrak{R} \lambda>-b$ and $D(\lambda, \gamma)=0$, it follows from (4.7) that (3.1) has a solution with $\|h\|_{a}$ and $\|v\|_{a}$ finite. Conversely, if (3.1) has such a nontrivial solution, it is rather straightforward to show that $e^{a s} y^{+}(s, \lambda, \gamma)=o(1)$ as $s \rightarrow \pm \infty$, utilizing results from [62] regarding the theory of asymptotic behavior of solutions of systems of ordinary differential equations with asymptotically constant coefficients. By Proposition 4.1, it follows that $D(\lambda, \gamma)=0$.

\subsection{The Evans function for the KdV equation}

The Evans function for the eigenvalue problems above, taken in the KdV scaling, will be related to the Evans function for the $\mathrm{KdV}$ equation itself, so it is appropriate to discuss the latter at this point. In Section 3 we observed how the scaled eigenvalue equations in (3.3), for Boussinesq solitary waves, converge formally to the eigenvalue problem for KdV solitary waves:

$$
\Lambda H+\partial_{\xi}^{3} H-\partial_{\xi} H+\partial_{\xi}(\Theta H)=0, \quad \text { where } \Theta(\xi)=3 \operatorname{sech}^{2}\left(\frac{1}{2} \xi\right)
$$

One may associate an Evans function $D_{\mathrm{KdV}}(\Lambda)$ with this equation, and it may be characterized as follows. See $[17,60]$ for the proofs. The characteristic polynomial that we associate with (4.10) is

$$
\mathscr{P}_{\mathrm{KdV}}(\mu)=\Lambda+\mu^{3}-\mu .
$$

For $\Lambda$ in the complex plane, except for real $\Lambda \leq-\sqrt{\frac{4}{27}}$, it turns out that $\mathscr{P}_{\text {KdV }}$ has a unique zero of smallest real part, which we denote by $\mu=\kappa_{1}(\Lambda)$. Equation (4.10) has a unique solution $H^{+}(\xi, \Lambda)$ satisfying $H^{+}(\xi, \Lambda) \sim e^{\kappa_{1} \xi}$ as $\xi \rightarrow+\infty$. The Evans function $D_{\mathrm{KdV}}(\Lambda)$ is the "transmission coefficient" 
for this solution, with the property that

$$
H^{+}(\xi, \Lambda) \sim D_{\mathrm{KdV}}(\Lambda) e^{\kappa_{1} \xi} \quad \text { as } \xi \rightarrow-\infty .
$$

The following theorem is a summary of the properties of $D_{\mathrm{KdV}}$.

THEOREM 4.6. (i) The domain, $\Omega_{K d V}$, of $D_{K d V}$ is equal to the complex plane, cut along the negative real axis from $-\infty$ to $-\Lambda_{*}(0)=-\sqrt{\frac{4}{27}}$.

(ii) The Evans function $D_{K d V}(\Lambda)$ is given by the explicit formula

$$
D_{K d V}(\Lambda)=\left(\frac{\kappa_{1}+1}{\kappa_{1}-1}\right)^{2},
$$

where $\mu=\kappa_{1}(\Lambda)$ is the root of smallest real part of Equation (4.11).

(iii) The only point at which $D_{K d V}(\Lambda)$ vanishes is $\Lambda=0$, which is a zero of order two.

(iv) $D_{K d V}(\Lambda) \rightarrow 1$ as $|\Lambda| \rightarrow \infty$ with $\Lambda \in \Omega_{K d V}$.

Remark 4.7: The zeros of the cubic polynomial, $\mathscr{P}_{\mathrm{KdV}}$, may be given explicitly as

$$
\begin{gathered}
\mu=r+\frac{1}{3 r}, \quad \text { where } r=\theta^{1 / 3} e^{2 \pi i k / 3}, k=0,1,2, \\
\text { and } \theta=-\frac{1}{2}\left(\Lambda+\sqrt{\Lambda^{2}-\frac{4}{27}}\right)
\end{gathered}
$$

For $\Lambda<-\sqrt{\frac{4}{27}}$, the zero of smallest real part is not unique. At $\Lambda=-\sqrt{\frac{4}{27}}$, the two smallest zeros are equal, having the value $\mu=-1 / \sqrt{3}$.

Remark 4.8: The fact that $\Lambda=0$ is a zero of order two is closely connected with the existence of a two-parameter family of solitary wave solutions for the $\mathrm{KdV}$ equation (2.4). For a full discussion, see [17].

\subsection{The Evans function in the KdV scaling}

In Section 4.1 we studied the Evans function associated with each of the eigenvalue problems in (3.1). In an entirely analogous way, we may introduce the Evans function $D_{*}(\Lambda, \gamma)$ associated with each of the scaled eigenvalue problems in (3.3). The function $D_{*}$ is directly related to $D$, but what is of interest is the direct relation of $D_{*}$ to $D_{\mathrm{KdV}}$ in the limit $\gamma \rightarrow 0$.

Each of the equations in (3.3) is written by standard reduction as a first-order system

$$
\frac{d Y}{d \xi}=A_{*}(\xi, \Lambda, \gamma) Y
$$


The reduction is defined for each system in (3.3) respectively by

$$
Y^{t}=\left(H, \partial_{\xi} H, \partial_{\xi}^{2} H, \partial_{\xi}^{3} H\right),\left(H, \partial_{\xi} H, \partial_{\xi}^{2} H, V\right) \text {, or }\left(V, \partial_{\xi} V, \partial_{\xi}^{2} V, H\right) \text {. }
$$

Let $A_{*}^{\infty}(\Lambda, \gamma)=\lim _{\xi \rightarrow \pm \infty} A_{*}(\xi, \Lambda, \gamma)$. The characteristic polynomial of $A_{*}^{\infty}(\Lambda, \gamma)$ works out to be

$$
\begin{aligned}
\mathscr{P}_{*}(\nu) & =\mathscr{P}_{*}(\nu, \Lambda, \gamma)=\gamma^{-4} \mathscr{P}(\gamma \nu ; \lambda, \gamma) \\
& =\nu \mathscr{P}_{\mathrm{KdV}}(\nu)-\gamma^{2}\left(\nu^{3} \Lambda+\frac{1}{4} \Lambda^{2}\right)+\frac{1}{4} \gamma^{4} \nu^{2} \Lambda^{2}
\end{aligned}
$$

(As usual, $\lambda=\frac{1}{2} \gamma^{3} \Lambda$.) The zeros of $\mathscr{P}_{*}(\nu)$ will be denoted $\nu_{j}$. Clearly, these are related to the zeros $\mu_{j}$ of $\mathscr{P}$ and the zeros $\kappa_{j}$ of $\mathscr{P}_{\mathrm{KdV}}$. We have

$$
\nu_{j}(\Lambda, \gamma)=\gamma^{-1} \mu_{j}(\lambda, \gamma) \quad \text { for } \gamma>0
$$

For $\gamma=0$, however, the zeros of $\mathscr{P}_{*}$ simply consist of the three zeros $\kappa_{j}$ of $\mathscr{P}_{\mathrm{KdV}}$, together with the value $\nu=0$.

To define an Evans function for (4.14), we need to verify that hypotheses corresponding to $\mathrm{H} 1-\mathrm{H} 4$ hold on an appropriate simply connected domain $\Omega_{*}$. Hypotheses $\mathrm{H} 1-\mathrm{H} 3$ will be easy to verify once we describe in Section 7 the specific structure of the matrices arising from the eigenvalue problems in Section 3. To verify H4, however, we must identify a domain in which we may always label the eigenvalues of $A_{*}^{\infty}(\Lambda, \gamma)$ so that

$$
\mathfrak{R} \nu_{1}(\Lambda, \gamma)<\min _{j>1} \mathfrak{R} \nu_{j}(\Lambda, \gamma)
$$

As an immediate consequence of Proposition 4.3 and the behavior of the zeros $\kappa_{j}$ that was discussed following (4.11), an appropriate domain is given as follows. Compare Proposition 4.3.

Proposition 4.9. For $0<\gamma \leq \gamma_{0}$, define the set $\Omega_{*}^{\gamma}=2 \gamma^{-3} \Omega^{\gamma}$, so that

$$
\Omega_{*}^{\gamma}=\left\{\Lambda \mid \Re \lambda \geq-2 \varepsilon_{0} / \gamma^{3}\right\} \backslash\left(-\infty,-\Lambda_{*}(\gamma)\right]
$$

For $\gamma=0$ define $\Omega_{*}^{0}=\Omega_{K d V}=\mathbb{C} \backslash\left(-\infty,-\sqrt{\frac{4}{27}}\right]$. Then if $0 \leq \gamma \leq \gamma_{0}$, for all $\Lambda \in \Omega_{*}^{\gamma}$ the matrix $A_{*}^{\infty}(\Lambda, \gamma)$ has a unique eigenvalue of smallest real part, which is simple.

Just as in Section 4.32, for $\Lambda \in \Omega_{*}^{\gamma}$ Equation (4.14) has a unique solution $Y^{+}(\xi, \Lambda, \gamma)$, which is $o\left(e^{\nu * \xi}\right)$ as $\xi \rightarrow+\infty$, with the normalization

$$
Y^{+}(\xi, \Lambda, \gamma) \sim e^{\nu_{1} \xi} \mathbf{v}_{1^{*}} \quad \text { as } \xi \rightarrow+\infty
$$


Here $\mathbf{v}_{1^{*}}$ is an eigenvector of $A_{*}^{\infty}$, which we take in the form $\mathbf{v}_{1^{*}}=\left(1, \nu_{1}, \nu_{1}^{2}, v_{*}\right)$. Then the Evans function $D_{*}(\Lambda, \gamma)$ is characterized by

$$
Y^{+}(\xi, \Lambda, \gamma) \sim D_{*}(\Lambda, \gamma) e^{\nu_{1} \xi} \mathbf{v}_{1^{*}} \quad \text { as } \xi \rightarrow-\infty .
$$

Note that by Proposition 4.9, the domain of $D_{*}(\Lambda, \gamma), \Omega_{*}^{\gamma}$, approaches $\Omega_{\mathrm{KdV}}$, the domain of $D_{\mathrm{KdV}}(\Lambda)$, as $\gamma \rightarrow 0$. In a manner entirely analogous to the proof of Proposition 4.4, one may prove:

Proposition 4.10. The Evans function $D_{*}(\Lambda, \gamma)$ is analytic in $\Lambda$ and jointly continuous for $(\Lambda, \gamma)$ in the set

$$
\Omega_{*} \equiv\left\{(\Lambda, \gamma) \mid 0 \leq \gamma \leq \gamma_{0} \text { and } \Lambda \in \Omega_{*}^{\gamma}\right\} .
$$

\subsection{Relations among the Evans functions}

For each system in (3.1) and the corresponding scaled system in (3.3), the Evans functions $D(\lambda, \gamma)$ and $D_{*}(\Lambda, \gamma)$ are related to each other and $D_{\mathrm{KdV}}$ in the following way.

THEOREM 4.11. With $\lambda=\frac{1}{2} \gamma^{3} \Lambda$, we have

$$
D_{*}(\Lambda, \gamma)=D(\lambda, \gamma) \text { for } \gamma>0 \text { and } D_{*}(\Lambda, 0)=D_{K d V}(\Lambda) \text {. }
$$

Proof: Consider the case $0<\gamma<1$. Because of the relationships $\mu_{1}=\gamma \nu_{1}$ and $\xi=\gamma s$, we have $\mu_{1} s=\nu_{1} \xi$. From (3.2), (4.4), and (4.19) it then follows that since

$$
y_{1}^{+}(s, \lambda, \gamma) \sim e^{\mu_{1} s} \quad \text { and } \quad Y_{1}^{+}(\xi, \Lambda, \gamma) \sim e^{\nu_{1} \xi}
$$

as $s, \xi \rightarrow+\infty$, it must be (recalling (4.7), (4.15), and (3.2)) that

$$
y_{1}^{+}(s, \lambda, \gamma)=Y_{1}^{+}(\xi, \Lambda, \gamma)
$$

Then $D_{*}(\Lambda, \gamma)=D(\lambda, \gamma)$, because of the characterizations of these Evans functions in (4.5) and (4.20).

In the case $\gamma=0$, we note that $\nu_{1}(\Lambda, 0)=\kappa_{1}(\Lambda)$ (see (4.16) and Theorem 4.6). Furthermore, the solution $\mathrm{H}^{+}$of the linearized $\mathrm{KdV}$ equation (4.10), which satisfies $H^{+}(\xi, \Lambda) \sim e^{\kappa_{1} \xi}$ as $\xi \rightarrow+\infty$, yields a valid solution $Y$ of the system derived from (3.3) with $Y_{1}(\xi, \Lambda, 0)=H^{+}(\xi, \Lambda)$. It follows that $Y_{1}^{+}=$ $H^{+}$. Because of the characterization of Evans functions in (4.12) and (4.20), we deduce that $D_{*}(\Lambda, 0)=D_{\mathrm{KdV}}(\Lambda)$. 


\section{Main theorems on absence of nonzero eigenvalues}

A central technical result of this article is the following. It concerns the convergence of the Evans function $D_{*}$ for each scaled eigenvalue problem in (3.3) to the Evans function $D_{\mathrm{KdV}}$ for the $\mathrm{KdV}$ eigenvalue problem in (3.4), in the limit $\gamma \rightarrow 0$. The convergence is uniform in the whole domain $\Omega_{*}^{\gamma}$ outside a sectorial neighborhood of the branch cut along the negative real axis. To describe the domain of convergence, recall that $\varepsilon_{0}=0.7$ from Proposition 4.3. Let $\sigma>0$ be an arbitrary small number, and define regions in the complex plane by

$$
\begin{gathered}
\mathscr{S}^{0}=\left\{\Lambda \mid \text { Either }|\arg (\Lambda)| \leq \pi-\sigma \text { or } \mathfrak{R} \lambda \geq-\sqrt{\frac{4}{27}}+\sigma\right\}, \\
\mathscr{S}^{\gamma}=\mathscr{S}^{0} \cap\left\{\Lambda \mid \Re \lambda \geq-2 \varepsilon_{0} / \gamma^{3}\right\}, \quad 0<\gamma<1 .
\end{gathered}
$$

(See Figure 1.)

THEOREM 5.1 (Convergence of the Evans Function). Fix $\sigma>0$, and define $\mathscr{S}^{\gamma}$ as above. Then

$$
\sup _{\Lambda \in \mathscr{S}^{\gamma}}\left|D_{*}(\Lambda, \gamma)-D_{\mathrm{KdV}}(\Lambda)\right| \rightarrow 0 \quad \text { as } \gamma \rightarrow 0
$$

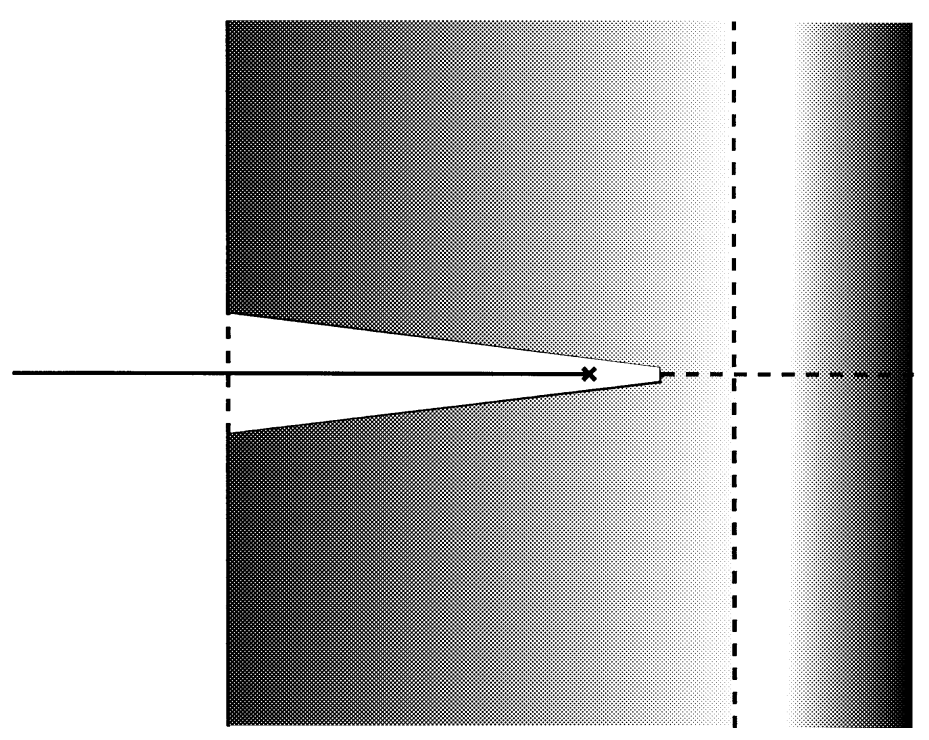

Figure 1. Domain of uniform convergence. 
In Section 9, we prove that for $0<\gamma \leq \gamma_{0}, \Lambda=0$ is a zero of $D_{*}(\Lambda, \gamma)$ of order at least two, a fact that is related to the symmetries of spatial translation and changing wave speed for the family of solitary waves. From this fact and Theorems 5.1 and 4.6, one may easily deduce the following, using Rouché's theorem on zeros of analytic functions under perturbation.

Corollary 5.2 (Zeros of $D_{*}$ ). For sufficiently small $\gamma>0$, the only zero of $D_{*}(\Lambda, \gamma)$ with $\Lambda \in \mathscr{S}^{\gamma}$ is $\Lambda=0$, which is a zero of order two exactly. Consequently

$$
D_{*}(\Lambda, \gamma)=D_{\mathrm{KdV}}(\Lambda)(1+o(1))
$$

as $\gamma \rightarrow 0$, uniformly for $\Lambda \in \mathscr{S}^{\gamma}$.

At this point, we may deduce our main result concerning the eigenvalue problems in Section 3, considered with respect to the exponentially weighted norm in (1.3). This result follows immediately from Corollary 5.2 and Proposition 4.5.

THEOREM 5.3. Suppose $a=c_{0} \gamma$, where $0<c_{0}<1 / \sqrt{3}$ and $\gamma>0$ is sufficiently small. Then there exists $b>0$, such that if $\lambda \neq 0$ with $\Re \lambda>-b$, then each eigenvalue problem in (3.1) has no nontrivial solution such that $\|h\|_{a}$ and $\|v\|_{a}$ are finite.

The proof of Theorem 5.1 and its corollary are organized as follows. In the rest of this section, we outline the proof of Theorem 5.1. In Section 6 we establish a number of fundamental properties of the roots $\mu_{j}$ of the characteristic polynomial $\mathscr{P}(\mu ; \lambda, \gamma)$, proving Lemma 4.2, Proposition 4.3 (which verifies hypothesis $\mathrm{H} 4$ ), and some auxiliary estimates. In Section 7 we describe the matrices $A$ and $A^{\infty}$ specifically in each case and verify the hypotheses $\mathrm{H} 1-\mathrm{H} 3$, which ensure that $D(\lambda, \gamma)$ is defined on $\Omega^{\gamma}$; also we obtain some asymptotic estimates for the eigenvectors of $A^{\infty}$. In Section 8 we explain the joint continuity of $D(\lambda, \gamma)$, and in Section 9 we show that $\lambda=0$ is a zero of order at least two, which yields Corollary 5.2. Finally in Section 10 we study the Evans function in two regimes corresponding to large values of $\lambda$ and $\Lambda$, finishing the results needed to prove Theorem 5.1.

\subsection{Four regimes for the eigenvalue parameter}

The proof of Theorem 5.1 involves the study of $D(\lambda, \gamma)$ in four overlapping regimes for the values of $\lambda=\frac{1}{2} \gamma^{3} \Lambda$ and $\gamma$. For some suitably small $\delta>0$, which shall be fixed later, these regimes correspond to the sets $S_{1}, \ldots, S_{4}$ defined as follows.

Regime I: $\quad S_{1}=\left\{\Lambda \| \Lambda b v \leq 2 \delta^{-1}\right\} \cap \mathscr{S}^{\gamma}$ 
In this regime, $\Lambda$ is bounded and is bounded away from the branch cut in the domain of $D_{*}(\Lambda, \gamma)$ on the negative real axis. It turns out to be surprisingly easy to study the limit $\gamma \rightarrow 0$ in this regime.

Proposition 5.4. Let $\delta>0$ be given. Then

$$
\lim _{\gamma \rightarrow 0} \sup _{\Lambda \in S_{1}}\left|D_{*}(\Lambda, \gamma)-D_{\mathrm{KdV}}(\Lambda)\right|=0
$$

Proof: This is a simple consequence of the joint continuity of $D_{*}(\Lambda, \gamma)$, the fact that $D_{*}(\Lambda, 0)=D_{\mathrm{KdV}}(\Lambda)$, and the fact that $S_{1}$ is a fixed compact subset of the domain $\Omega_{*}^{\gamma}$ for sufficiently small $\gamma \geq 0$.

In the remaining three regimes, the idea is that since $D_{\mathrm{KdV}}(\Lambda) \rightarrow 1$ as $|\Lambda| \rightarrow \infty$, we wish to show that $D(\lambda, \gamma)-1$ is small if $\delta$ and $\gamma$ are sufficiently small. In Regimes II and IV, this is accomplished using Lemma 10.1, which is related to the results of $[60$, Section $1(\mathrm{~g})]$, concerning the behavior of $D(\lambda)$ for large $\lambda$. To use this lemma, some careful estimates of the roots $\mu_{j}$ and their associated eigenvectors will be needed.

Regime II: $\quad S_{2}=\left\{\lambda\left|\gamma^{3} \delta^{-1} \leq\right| \lambda \mid \leq \delta\right.$ and $\left.|\arg \lambda| \leq \pi-\sigma\right\}$

Regime II represents a "crossover" regime; $\Lambda$ is large but $\lambda$ is small. In this regime we obtain the following estimate.

Proposition 5.5. There exists positive constants $C_{2}, \delta_{2}$, and $\gamma_{2}$, such that for $0 \leq \gamma \leq \gamma_{2}, 0<\delta \leq \delta_{2}$, we have

$$
\sup _{\lambda \in S_{2}}\left|D_{*}(\Lambda, \gamma)-1\right|<C_{2} \delta^{1 / 3}, \quad \text { where } \frac{1}{2} \gamma^{3} \Lambda=\lambda
$$

Regime III: $\quad S_{3}=\left\{\lambda|\delta \leq| \lambda \mid \leq \delta^{-1}\right.$ and $\Re \lambda \geq-\varepsilon_{0}$ and $\left.|\arg \lambda| \leq \pi-\sigma\right\}$

In Regime III, $\lambda$ lies in a fixed compact set bounded away from infinity and from the branch cut on the negative real axis. From Proposition 4.4, we infer that $D(\lambda, \gamma)$ is jointly continuous in $(\lambda, \gamma)$ for $(\lambda, \gamma) \in S_{3} \times\left[0, \gamma_{0}\right]$. Since $D(\lambda, 0)$ is the Evans function for a problem in the form (4.1) with constant coefficients, the characterization (4.5) immediately yields

$$
D(\lambda, 0) \equiv 1
$$


Therefore we obtain the following.

Proposition 5.6. Let $\delta>0$ be given. Then

$$
\lim _{\gamma \rightarrow 0} \sup _{\lambda \in S_{3}}|D(\lambda, \gamma)-1|=0
$$

Regime IV: $S_{4}=\left\{\lambda\left|\delta^{-1} \leq\right| \lambda \mid\right.$ and $\left.\mathfrak{R} \lambda \geq-\varepsilon_{0}\right\}$

Here $|\lambda|$ is large. Curiously, this regime is technically by far the most difficult to treat. The strategy is similar to that for Regime II. We need to prove the following result, whose proof is concluded in Section 10.

Proposition 5.7. There exist positive constants $C_{4}, \gamma_{4}$, and $\delta_{4}$, such that if $0<\delta \leq \delta_{4}$ and $0<\gamma \leq \gamma_{4}$, then we have

$$
\sup _{\lambda \in S_{4}}|D(\lambda, \gamma)-1| \leq C_{4} \gamma
$$

Proof of Theorem 5.1: Let $\varepsilon>0$. Then by invoking Theorem 4.6 and Propositions 5.5 and 5.7, we may assert that there exist $\delta>0$ and $\gamma_{1}>0$ such that

$$
\begin{aligned}
\left|D_{\mathrm{KdV}}(\Lambda)-1\right|<\frac{1}{2} \varepsilon & \text { for }|\Lambda| \geq \delta^{-1}, \\
\sup _{\lambda \in S_{2}}\left|D_{*}(\Lambda, \gamma)-1\right|<\frac{1}{2} \varepsilon & \text { for } \lambda=\frac{1}{2} \gamma^{3} \Lambda, 0<\gamma \leq \gamma_{1}, \\
\sup _{\lambda \in S_{4}}|D(\lambda, \gamma)-1|<\frac{1}{2} \varepsilon & \text { for } 0<\gamma \leq \gamma_{1} .
\end{aligned}
$$

With $\delta$ now fixed, by Proposition 5.6 we have

$$
\sup _{\lambda \in S_{3}}|D(\lambda, \gamma)-1|<\frac{1}{2} \varepsilon
$$

for $\gamma$ sufficiently small. Finally, using Proposition 5.4 and the fact that $\bigcup_{j=1}^{4} S_{j}=\mathscr{S}^{\gamma}$, we deduce that

$$
\sup _{\Lambda \in \mathscr{S}^{\gamma}}\left|D_{*}(\Lambda, \gamma)-D_{\mathrm{KdV}}(\Lambda)\right|<\varepsilon
$$

for $\gamma$ sufficiently small. This proves Theorem 5.1. 


\section{Characteristic roots}

In this section we study the characteristic roots $\mu_{j}(\lambda, \gamma)$ of the polynomial given in (4.8), namely

$$
\mathscr{P}(\mu ; \lambda, \gamma)=\left(\mu^{2}-1\right)(\lambda-\mu)^{2}+\mu^{2} / c^{2} \text {. }
$$

(Recall $c=\sqrt{1 /\left(1-\gamma^{2}\right)}$.) We prove Lemma 4.2 and Proposition 4.3 and develop estimates for the roots $\mu_{j}$ that are needed in Regimes II and IV described in Section 5.2.

Proof of Lemma 4.2(a): Note that $\mathscr{P}(\mu)$ never vanishes for $\mu= \pm 1$, and therefore, satisfying the equation $\mathscr{P}=0$ is equivalent to satisfying one of the equations

$$
\lambda=\mathscr{P}_{+}(\mu, \gamma) \equiv \mu+\frac{\mu}{c \sqrt{1-\mu^{2}}}, \quad \lambda=\mathscr{P}_{-}(\mu, \gamma) \equiv \mu-\frac{\mu}{c \sqrt{1-\mu^{2}}}
$$

(Below, we frequently suppress the dependence of $\mathscr{P}_{ \pm}$upon $\gamma$.) Clearly, if $\mathfrak{i} \lambda \neq 0$, there are no solutions $\mu$ of $\left(6.1_{ \pm}\right)$with $\mathfrak{R} \mu=0$. Therefore, the number of solutions of $\left(6.1_{ \pm}\right)$in the right half-plane or left half-plane may change only if the sign of $\mathfrak{R}^{ \pm} \lambda$ changes.

To calculate this number, first note that $\mathscr{P}_{-}( \pm \gamma, \gamma)=0$. Second, it is easy to check that the functions $t \mapsto-i \mathscr{P}_{ \pm}(i t, \gamma)$ are both strictly increasing functions, which map the real line onto itself. So, for $\mathfrak{R} \lambda=0$, there are exactly two zeros $\mu$ with $\Re \mu=0$. From these two facts it follows that if $\Re \lambda=0$, then there is one root $\mu$ in the left half-plane and one root in the right half-plane.

Finally, since $\partial_{\mu} \mathscr{P}_{ \pm}(0, \gamma)=1 \pm 1 / c>0$, as $\lambda$ moves into the right (respectively the left) half-plane, so do both imaginary roots. The lemma follows.

Proof of Proposition 4.3: From Lemma 4.2(a), we infer that it suffices to establish the result for $\Re \lambda<0$. For real values of $\lambda$, the result is comparatively easy to prove. Suppose $\lambda<0$. The function $\mathscr{P}_{+}$monotonically maps $\mu$ in $(-1,1)$ onto the real line, so Equation $\left(6.1_{+}\right)$has a unique solution, which we label $\mu_{4}$, and this solution clearly satisfies $\lambda<\mu_{4}<0$. The function $\mathscr{P}_{-}$ is odd in $\mu$, vanishes at $\mu=0$ and $-\gamma$, and is strictly convex on $(-1,0)$. It has a minimum at $\mu_{m}=-\left(1-c^{-2 / 3}\right)^{1 / 2}$, with minimum value equal to 
$-\lambda_{*}(\gamma)$, where

$$
\mathscr{P}_{-}\left(\mu_{m}\right)=-\lambda_{*}(\gamma)=-\frac{1}{2} \gamma^{3} \Lambda_{*}(\gamma)=-\left(1-c^{-2 / 3}\right)^{3 / 2}
$$

(See Figure 2.) We note that as $\gamma \rightarrow 0, \mu_{m} \sim-\gamma / \sqrt{3}$ and $\lambda_{*}(\gamma) \sim$ $-\gamma^{3} / \sqrt{27}$. For $-\lambda_{*}(\gamma)<\lambda<0$, Equation $\left(6.1_{-}\right)$has three real solutions that satisfy

$$
\mu_{1}<\mu_{2}<\lambda<0<\mu_{3}
$$

It remains to study the situation when $\mathfrak{J} \lambda>0$, since the case $\mathfrak{J} \lambda<0$ can be reduced to this one by taking the complex conjugate. We proceed via a series of lemmas, first analyzing Equation (6.1 $)$.

Lemma 6.1. Suppose $-1<\mathfrak{R} \lambda<0$ and $\mathfrak{S} \lambda>0$. Then (6.1 $1_{+}$) has a unique solution, labeled $\mu=\mu_{4}$, and this solution satisfies

$$
\mathfrak{R} \lambda<\mathfrak{R} \mu_{4}<0, \quad 0<\mathfrak{\Im} \mu_{4}<\mathfrak{\Im} \lambda
$$

Proof: Consider the function defined by

$$
Q(\mu)=\mu / \sqrt{1-\mu^{2}} .
$$

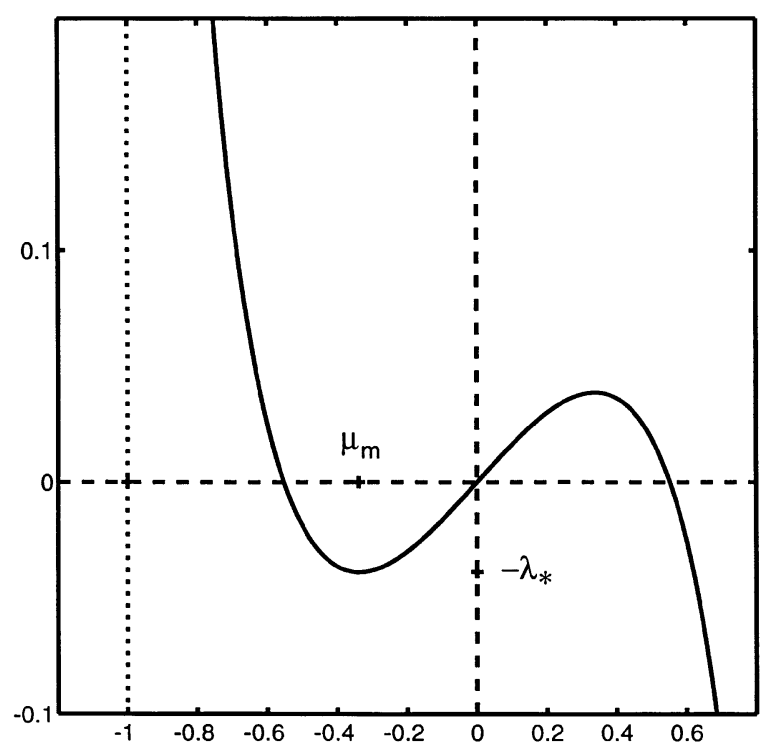

Figure 2. $\quad P_{-}(t)$ vs $t(c=1.2)$. 
Then $Q$ is analytic for $\mu$ in the half-plane $\Re \mu \leq 0$ minus the cut $(-\infty,-1]$, is odd in $\mu$, and is bounded outside any neighborhood of the set $\{-1,1\}$. Also, note that $Q(\mu)^{2}$ is real only if $\mu^{2}$ is. It follows that $Q$ leaves each quadrant of the complex plane invariant. That is, the sign of $\mathfrak{R} Q(\mu)$ (resp. the sign of $\mathfrak{I} Q(\mu)$ ) agrees with the sign of $\mathfrak{\imath} \mu$ (resp. the sign of $\mathfrak{I} \mu$ ).

From this last property of $Q$, we may infer that, given $\lambda$ as supposed, any solution of $\left(6.1_{+}\right)$must satisfy (6.3). To see that a unique solution exists, consider the rectangle

$$
B=\{\mu \mid \mathfrak{R} \lambda<\mathfrak{R} \mu<0,0<\mathfrak{S} \mu<\mathfrak{S} \lambda\} .
$$

Because $Q$ leaves each quadrant invariant, the image of this rectangle under $\mathscr{P}_{+}$contains the rectangle, i.e., $\mathscr{P}_{+}(B) \supset B$, and furthermore, one may infer that the image of the boundary of the rectangle, $\mathscr{P}_{+}(\partial B)$, has winding number 1 about $\lambda$. Therefore a unique solution of $\left(6.1_{+}\right)$exists.

Now we turn our attention to Equation (6.1_ ). Because of the equivalence between (6.1) and the polynomial equation $\mathscr{P}=0$, it follows that if $-1<\Re \lambda<0$, Equation (6.1_) has three solutions. It turns out that these solutions lie in three different quadrants. To characterize them, we use the following result.

LEMMA 6.2. Suppose $-1<\Re \lambda<1$, that $\lambda^{2}$ is not real, and that $\lambda$ is not in the fourth quadrant of the complex plane. Then there exists a unique $\mu=\hat{\mu}(\lambda)$ lying in the third quadrant, which satisfies (6.1_).

Proof: We show that for certain contours $\Gamma$, which consist of a sufficiently large quarter-circle in the third quadrant, with a small semicircle "cut out" near $\mu=-1$, the winding number of the image $\mathscr{P}_{-}(\Gamma)$ about $\lambda$ is 1 . This proves that $\left(6.1_{-}\right)$has a unique solution inside $\Gamma$.

Let $B(z, r)$ denote the disk in the complex plane with center $z$, radius $r$. For $\varepsilon>0$ sufficiently small, consider the set obtained by intersecting $B\left(0, \varepsilon^{-1}\right) \backslash B(-1, \varepsilon)$ with the third quadrant. The contour $\Gamma$ shall be the boundary of this set. (See Figure 3a.) That is,

$$
\Gamma=\partial\left\{\mu|\Re \mu<0, \mathfrak{I} \mu<0,| \mu\left|<\varepsilon^{-1},\right| \mu+1 \mid>\varepsilon\right\}
$$

The contour $\Gamma$ is the union of five curves:

$$
\begin{aligned}
& \Gamma_{1}=\{t \mid 0 \geq t \geq-1+\varepsilon\}, \quad \Gamma_{2}=\left\{-1+\varepsilon e^{-i t} \mid 0 \leq t \leq \pi\right\}, \\
& \Gamma_{3}=\left\{t-0 i \mid-1-\varepsilon \geq t \geq-\varepsilon^{-1}\right\}, \\
& \Gamma_{4}=\left\{-\varepsilon^{-1} e^{i t} \mid 0 \leq t \leq \frac{1}{2} \pi\right\}, \quad \Gamma_{5}=\left\{i t \mid-\varepsilon^{-1} \leq t \leq 0\right\} .
\end{aligned}
$$




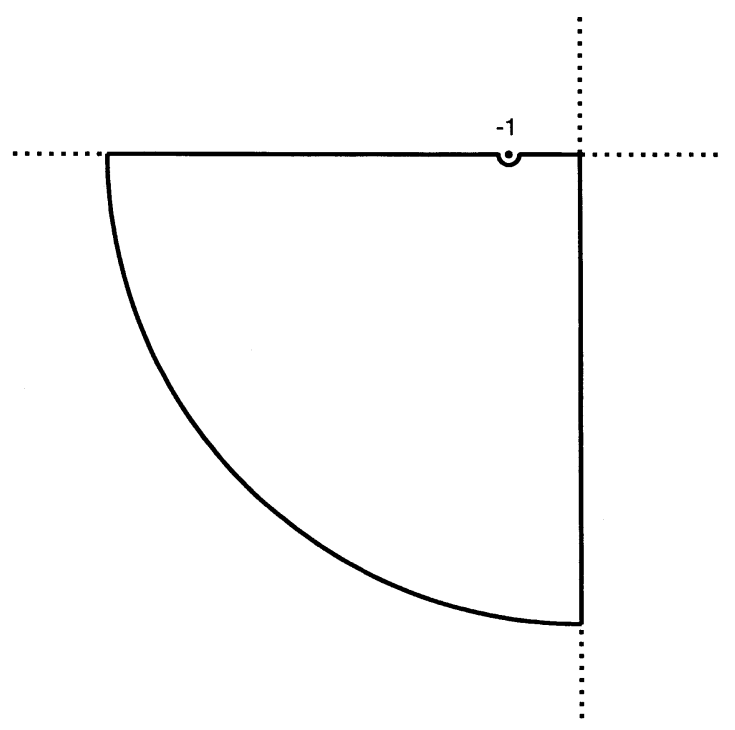

(a)

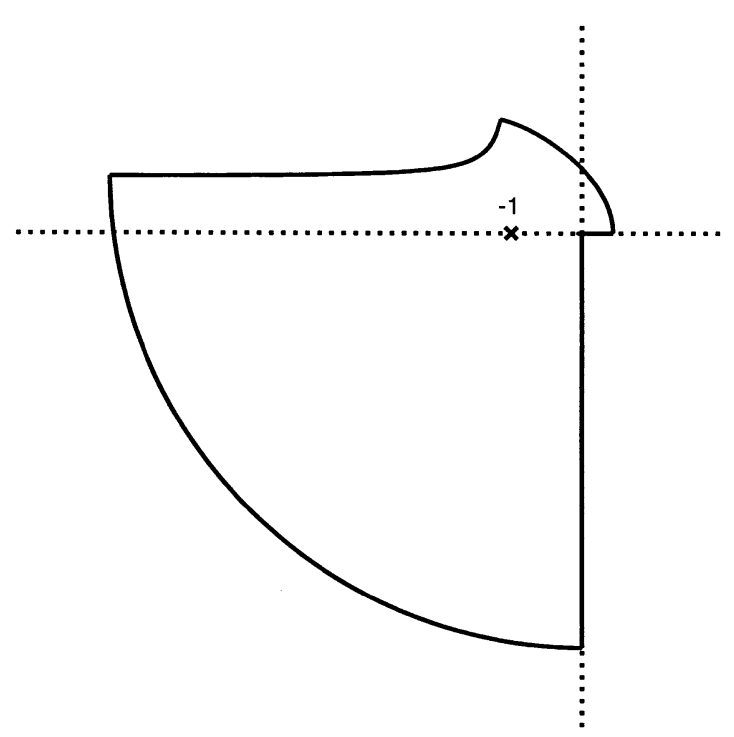

(b)

Figure 3. (a) The contour $\Gamma(\varepsilon=0.15)$. (b) Image of $\Gamma(c=1.25)$. 
To compute the winding number of $\mathscr{P}_{-}(\Gamma)$ about $\lambda$, we make the following observations: First, $\mathscr{P}_{-}\left(\Gamma_{1}\right)$ lies on the real axis. For $\mu \in \Gamma_{2}$, let $\mu=-1+$ $\varepsilon e^{-i t}$, where $0 \leq t \leq \pi$. Then we compute

$\mathscr{P}_{-}\left(-1+\varepsilon e^{-i t}\right)$

$$
=\left(-1+\varepsilon e^{-i t}\right)\left(1-\frac{e^{i t / 2}}{c \sqrt{2 \varepsilon}}\left(1-\frac{1}{2} \varepsilon e^{-i t}\right)^{-1 / 2}\right)=\frac{e^{i t / 2}}{c \sqrt{2 \varepsilon}}-1+O(\sqrt{\varepsilon}) .
$$

For $\mu \in \Gamma_{3}$, we have $\mathfrak{R} \mathscr{P}_{-}(\mu)<-1$, since

$$
\mathscr{P}_{-}(t+0 i)=t+\frac{i t}{c \sqrt{t^{2}-1}} \quad \text { for } t<-1 \text {. }
$$

For $\mu \in \Gamma_{4}, \quad \mu$ lies in the third quadrant, so $Q(\mu)=-i\left(1-\mu^{-2}\right)^{-1 / 2}$. Therefore as $|\mu| \rightarrow \infty$ in the third quadrant, $\mathscr{P}_{-}(\mu)=\mu+i / c+o(1)$. Finally, for $\mu \in \Gamma_{5}, \mathfrak{R} \mathscr{P}_{-}(\mu)=0$.

With these observations, it is straightforward to deduce that, if $\lambda$ is fixed and satisfies the hypotheses of the Lemma, and $\varepsilon$ is sufficiently small, then the winding number of $\mathscr{P}_{-}(\Gamma)$ about $\lambda$ is 1 . (See Figure $3 b$.) This finishes the proof.

Now, assume $-1<\Re \lambda<0$ and $\mathfrak{I} \lambda>0$. We claim that the solutions of (6.1_ ) may be labeled so that the following relations hold:

$$
\begin{array}{lll}
\mathfrak{R} \mu_{1}<0, & \mathfrak{R} \mu_{2}<0, & \mathfrak{R} \mu_{3}>0, \\
\mathfrak{S} \mu_{1}<0, & \mathfrak{S} \mu_{2}>0, & \mathfrak{S} \mu_{3}<0 .
\end{array}
$$

To prove these relations, we exploit the symmetries $\mathscr{P}_{-}(-\mu)=\mathscr{P}_{-}(\mu)$, $\mathscr{P}_{-}(\bar{\mu})=\overline{\mathscr{P}_{-}(\mu)}$. It suffices to invoke Lemma 6.2 and define $\mu_{j}=\mu_{j}(\lambda, \gamma)$ for $j=1,2,3$ via

$$
\mu_{1}=\hat{\mu}(\lambda), \quad \mu_{2}=\overline{\hat{\mu}(\bar{\lambda})}, \quad \mu_{3}=-\overline{\hat{\mu}(-\bar{\lambda})}
$$

This establishes (6.4). Because $\mathfrak{R} Q(\mu)<0$ when $\mathfrak{R} \mu<0$, the relations in (6.3) and (6.4) imply that

$$
\mathfrak{R} \mu_{j}<\mathfrak{R} \lambda<\mathfrak{R} \mu_{4}<0<\mathfrak{R} \mu_{3}, \quad \text { for } j=1,2 .
$$

To prove Proposition 4.3, then, it will be enough to show that for $\gamma$ sufficiently small, if $\mathfrak{R} \lambda \geq-\varepsilon_{0}=-0.7$ and $\mathfrak{J} \lambda>0$, then

$$
\mathfrak{R} \mu_{1}<\mathfrak{R} \mu_{2} .
$$


By (6.5), proving this inequality is equivalent to showing that

$$
\mathfrak{R} \hat{\mu}(\lambda)<\mathfrak{R} \hat{\mu}(\bar{\lambda})
$$

Our strategy for proving this is as follows. For $a<0$, let $M_{a}$ be the open vertical half-strip defined by

$$
M_{a}=\{\mu \mid a<\mathfrak{R} \mu<0 \text { and } \mathfrak{\Im} \mu<0\} .
$$

As $a$ decreases from 0 , the images $\mathscr{P}_{-}\left(M_{a}\right)$ increase. For negative $a$ of sufficiently large magnitude, $M_{a}$ contains both $\hat{\mu}(\lambda)$ and $\hat{\mu}(\bar{\lambda})$, so that the image $\mathscr{P}_{-}\left(M_{a}\right)$ contains both $\lambda$ and $\bar{\lambda}$. To establish (6.7), what we must show is that as $a$ decreases, $\bar{\lambda}$ is "eaten" before $\lambda$ is. That is, we must show that there exists $a<0$ such that $\bar{\lambda} \in \mathscr{P}_{-}\left(M_{a}\right)$ but $\lambda \notin \mathscr{P}_{-}\left(M_{a}\right)$.

To carry out this strategy, we study the image of the vertical half-line on which $\Re \mu=a$ and $\mathfrak{S} \mu<0$. The results that we have obtained are restricted to the case when $a>-1$, however. See Figure $4 \mathrm{a}$ for a plot of the image $\mathscr{P}_{-}\left(M_{a}\right)$, for the sample case $c=1.2$, with $a=-0.7$. In Figure $4 \mathrm{~b}$ the image of the half-line corresponding to $a=-1$ is also indicated, as a dashed curve.

Lemma 6.3. Suppose $0 \leq \gamma<1$. Suppose $-1<a<0$, and define $\hat{\lambda}(t)=$ $\mathscr{P}_{-}(a-i t)$. Recall that $\mu_{m}=-\left(1-c^{2 / 3}\right)^{1 / 2}$ is the value at which $\mathscr{P}_{-}(\mu)$ attains its minimum for $-1<\mu \leq 0$. Then

(i) $\mathfrak{R} \hat{\lambda}(t)$ decreases as $t$ increases for $t>0$, and $\lim _{t \rightarrow \infty} \mathfrak{R} \hat{\lambda}(t)=a$.

(ii) If $\mu_{m} \leq a<0$, then $\mathfrak{J} \hat{\lambda}(t)$ decreases as $t$ increases for all $t>0$, and $\lim _{t \rightarrow \infty} \mathfrak{I} \hat{\lambda}(t)=-\infty$.

(iii) If $-1<a<\mu_{m}$, then for some $t_{a}>0$, $\mathfrak{\Im} \hat{\lambda}(t)$ increases on $\left(0, t_{a}\right)$, and decreases on $\left(t_{a}, \infty\right)$, and $\lim _{t \rightarrow \infty} \mathfrak{I} \hat{\lambda}(t)=-\infty$.

Proof: For $\mu=a-i t, \hat{\lambda}^{\prime}(t)=-i \mathscr{P}_{-}^{\prime}(\mu)$, so to prove (i) it suffices to show that $\mathfrak{S}_{\mathscr{P}_{-}^{\prime}}(\mu)<0$. We compute

$$
\mathscr{P}_{-}^{\prime}(\mu)=1-c^{-1}\left(1-\mu^{2}\right)^{-3 / 2}
$$

It is easy to check that if $-1<\mathfrak{R} \mu<0$ and $\mathfrak{\Im} \mu<0$, then $1-\mu^{2}$ lies in the fourth quadrant, and therefore $\mathfrak{\Im}_{\mathscr{P}_{-}^{\prime}}(\mu)<0$.

To prove (ii) and (iii), we must show that $\mathfrak{R} \mathscr{P}_{-}^{\prime}(a-i t)$ is always positive in case (ii) and changes sign once from negative to positive in case (iii). We have already seen that, for $t=0, \mathscr{P}_{-}^{\prime}(a)$ is in fact positive for $a \in\left(\mu_{m}, 0\right)$ and negative for $a \in\left(-1, \mu_{m}\right)$. Since from (6.8), $\mathfrak{\Im} \hat{\lambda}^{\prime}(t)=-\mathfrak{R} \mathscr{P}^{\prime}(a-i t) \rightarrow$ -1 as $t \rightarrow \infty$, it is enough to show that $\mathfrak{R} \mathscr{P}_{-}^{\prime}(a-i t)$ may change sign at most once. By (6.8), this is equivalent to showing that for $t \geq 0$ and $\mu=a-i t, \mathfrak{R}\left(1-\mu^{2}\right)^{-3 / 2}=c$ has at most one solution. 


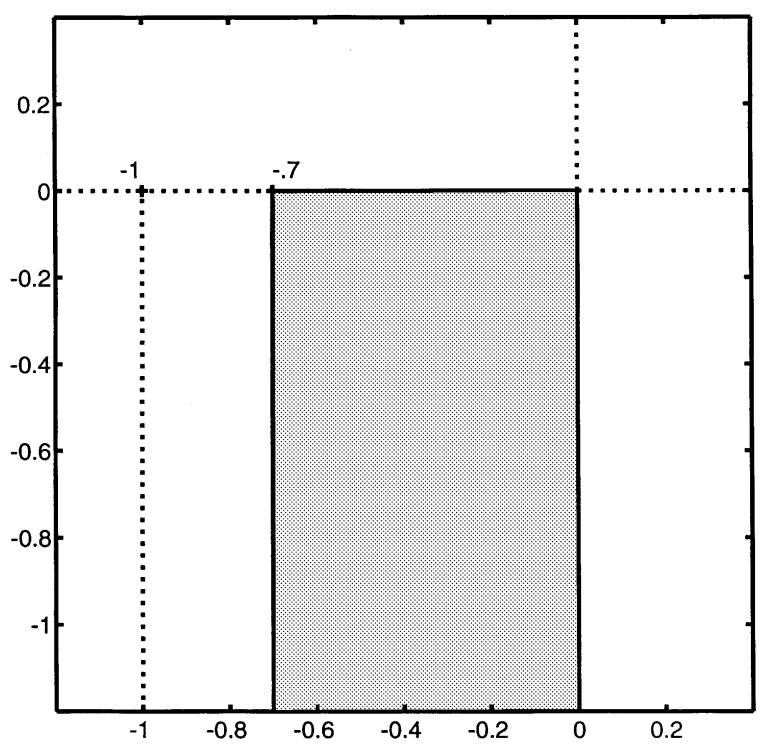

(a)

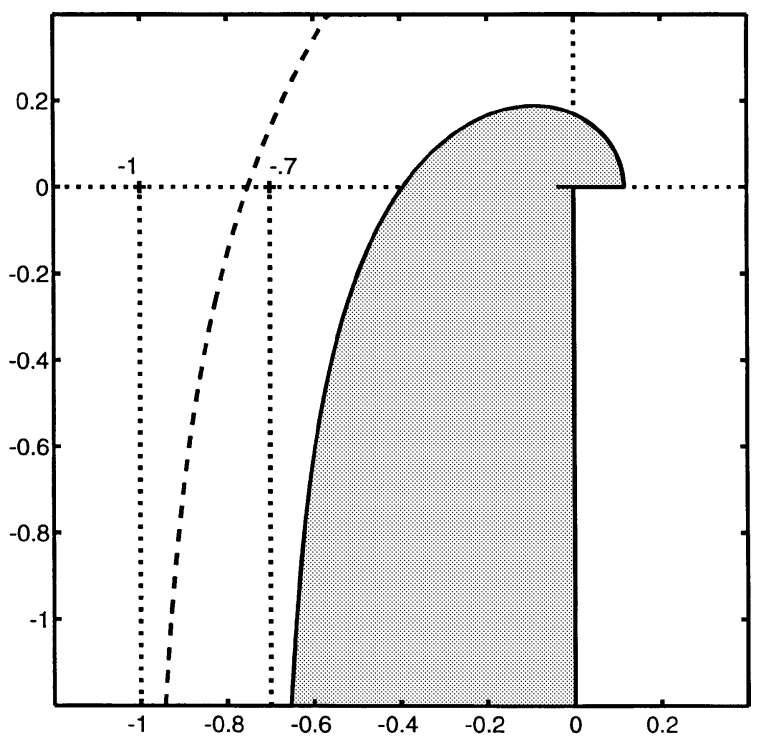

(b)

Figure 4. (a) Half strip $M_{a}$ with $a=-0.7$. (b) Image of half strip with $a=-0.7$ ( $c=1.2$ ). 
With $\mu=a-i t$, if we write $\left(1-\mu^{2}\right)^{-3 / 2}$ as $\rho(t) e^{i \theta(t)}$, it can be checked that $\rho(t)$ is strictly decreasing for $t>0$, and that $\theta(t)$ is increasing for $0 \leq t<t_{a}$ and decreasing for $t_{a}<t$, where $t_{a}=\sqrt{1-a^{2}}$. It follows that $g$ : $t \mapsto\left(1-\mu^{2}\right)^{-3 / 2}$ has decreasing real part for $0 \leq t \leq t_{a}$. Moreover, with $\mu_{a}=a-i t_{a}$, we find $\mathfrak{R}\left(1-\mu_{a}^{2}\right)^{-1}=\frac{1}{2}$, and one may compute that for all $t>0,(d / d t) \mathfrak{R}\left(1-\mu^{2}\right)^{-1}<0$. It follows that $\mathfrak{R} g(t)<\frac{1}{2}$ for $t \geq t_{a}$.

Note that $g(0)=\left(1-a^{2}\right)^{-3 / 2}$. For $-1<a<\mu_{m}$, we have $g(0)>c \geq 1$. From the above considerations, we infer that $\mathfrak{R}\left(1-\mu^{2}\right)^{-3 / 2}=c$ holds exactly once for $t>0$ if $-1<a<\mu_{m}$. This implies (iii). On the other hand, if $a \geq \mu_{m}$, we have $g(0) \leq c$ and therefore $\mathfrak{J} \hat{\lambda}(t)$ is decreasing for $t>0$, the statement in (ii).

Before we conclude the proof of Proposition 4.3, we observe that for the special values

$$
\mu_{0}=-\sqrt{0.96}-i \sqrt{0.84}, \quad \lambda_{0}=-\sqrt{0.54}, \quad \gamma=0,
$$

we have $\mathscr{P}\left(\mu_{0} ; \lambda_{0}, 0\right)=0$. In fact, for $(\lambda, \gamma)=\left(\lambda_{0}, 0\right)$ we have $\mu_{0}=\mu_{1}=\overline{\mu_{2}}$, and $\mu_{j}=\sqrt{0.06} \pm 0.6$ for $j=3$ and 4. Clearly $\lambda_{0}<-0.7$, so for the value $a=a_{0}=-\sqrt{0.96}$, the segment $\left[-0.7,-\lambda_{*}(\gamma)\right)$ lies below the curve $\mathscr{P}{ }_{-}(a-$ it), and this remains true for $0<\gamma \leq \gamma_{0}$, for some sufficiently small $\gamma_{0}>0$.

Indeed, the segment $\left[-0.7,-\lambda_{*}(\gamma)\right)$ lies in the image $\mathscr{P}_{-}\left(M_{a_{0}}\right)$. It is not hard to prove this by computing a winding number. For $\varepsilon>0$ and $-1<a<0$, let $M_{a}^{\varepsilon}$ be the rectangle $M_{a}^{\varepsilon}=M_{a} \cap\left\{\mu \mid \mathfrak{S} \mu>-\varepsilon^{-1}\right\}$. For sufficiently small $\varepsilon$, if $\widetilde{\Im} \mu=-\varepsilon^{-1}$, then $\widetilde{S}_{\mathcal{P}}(\mu)<-\varepsilon^{-1}+2$. Also $\mathscr{P}_{-}(\mu) \geq-\lambda_{*}(\gamma)$ is real if $\mu \in[a, 0]$, and $\mathscr{P}_{-}(\mu)$ is purely imaginary if $\mu$ is. So it is easy to see that the winding number of the curve $\mathscr{P}_{-}\left(\partial M_{a_{0}}^{\varepsilon}\right)$ about any point of the segment $\left[-0.7,-\lambda_{*}(\gamma)\right)$ is one.

We are now ready to finish the proof of Proposition 4.3. Assume $0<\gamma \leq \gamma_{0}$ and $-0.7 \leq \mathfrak{R} \lambda<0$ with $\mathfrak{S} \lambda>0$. Suppose at first that $\mathfrak{R} \lambda \in\left[-0.7,-\lambda_{*}(\gamma)\right)$. Then there exists a complex number $\alpha+i \beta \in M_{a_{0}}$ such that $\mathfrak{R} \lambda=\mathscr{P}_{-}(\alpha+$ $i \beta$ ). Since $\mathfrak{\Im} \lambda>0$, from the results of Lemma 6.3, and the argument of the preceding paragraph, we have that $\bar{\lambda} \in \mathscr{P}_{-}\left(M_{\alpha}\right)$, but $\lambda \notin \mathscr{P}_{-}\left(M_{\alpha}\right)$. The inequality (6.7) follows.

The remaining case to consider is when $\mathfrak{R} \lambda \in\left[-\lambda_{*}(\gamma), 0\right)$. But this case is easier-we may take $a=\mu_{m}$ and deduce that the image $\mathscr{P}_{-}\left(M_{a}\right)$ lies entirely in the third quadrant and contains $\bar{\lambda}$ but not $\lambda$. So $\mathfrak{R} \mu_{1}<\mu_{m}<\Re \mu_{2}$, giving (6.7) in this case also. This finishes the proof of Proposition 4.3.

Remark: Numerical computations indicate that one may take $\varepsilon_{0}=-1$ in the statement of Proposition 4.3, for any $\gamma \in[0,1)$, but we have not found a proof.

Proof of Lemma 4.2(b): Let $a=c_{0} \gamma$ where $c_{0} \in(0,1 / \sqrt{3})$; then $\mu_{m}<-a$ $<0$ for sufficiently small $\gamma$. Choose $b$ so that $0<b<-\mathscr{P}(-a)$. Suppose 
$\Re \lambda>-b$. If $\lambda$ is real the desired result follows from Lemma 4.2(a) and the first part of the proof of Proposition 4.3. Without loss of generality we may assume $\mathfrak{I} \lambda>0$. By Lemma 6.3 and the last part of the proof of Proposition 4.3 , it is clear that if $-b<\mathfrak{R} \lambda<0$, then

$$
\mathfrak{R} \mu_{1}<-a<\mathfrak{R} \mu_{2},
$$

since $\mathscr{P}_{-}\left(M_{-a}\right)$ contains $\bar{\lambda}$ but not $\lambda$. Now (6.6) yields the desired conclusion for such $\lambda$. If $\mathfrak{R} \lambda \geq 0$ and $\mathfrak{I} \lambda>0$, then $\mathfrak{R} \mu_{1}<\mu_{m}<-a$ and $0 \leq \mathfrak{i} \mu_{j}$ for $j>1$. This finishes the proof.

Our next result establishes approximations to the roots $\mu_{j}$ in Regime II, where $|\Lambda|$ is large but $|\lambda|$ is small.

Proposition 6.4. Let $\mu_{j}=\mu_{j}(\lambda, \gamma)$ denote the four roots of the quartic equation $\mathscr{P}(\mu ; \lambda, \gamma)=0$.

(a) There exists a positive constant $\gamma_{2}$, such that for $(\lambda, \gamma)$ satisfying $\gamma^{3} \delta_{2}^{-1}<|\lambda|<\delta_{2}, 0 \leq \gamma \leq \gamma_{2}$ :

$$
\mu_{j}=(-2 c \lambda)^{1 / 3} e^{2 \pi i j / 3}(1+o(1)) \quad \text { for } j=1,2,3
$$

uniformly as $\delta_{2} \rightarrow 0^{+}$.

(b) For $|\lambda|<\delta$ sufficiently small,

$$
\mu_{4}=\frac{c \lambda}{c+1}\left(1+\mathscr{O}\left(\lambda^{3}\right)\right)
$$

uniformly in $\gamma \in[0,1)$.

Proof: To prove (6.9), write $\left(1-\mu^{2}\right)^{-1 / 2}=1+\frac{1}{2} \mu^{2}+\mu^{4} r(\mu)$ where $r(\mu)$ is analytic near zero. Then $\left(6.1_{-}\right)$is equivalent to

$$
\frac{1}{2} \mu^{3}+c \lambda=(c-1) \mu-\mu^{5} r(\mu) .
$$

For $j=1,2,3$, let $\nu_{j}=(-2 c \lambda)^{1 / 3} e^{2 \pi i j / 3}$ and seek a solution of (6.11) in the form $\mu=\nu_{j}(1-\beta)^{1 / 3}$. Then Equation (6.11) is equivalent to

$$
\beta=\frac{(c-1) \nu_{j}}{c \lambda}(1-\beta)^{1 / 3}+\frac{\nu_{j}^{5}}{c \lambda}(1-\beta)^{5 / 3} r\left(\nu_{j}(1-\beta)^{1 / 3}\right)
$$

Since $(c-1) \nu_{j} / c \lambda=O\left(\gamma^{2}|\lambda|^{-2 / 3}\right)$ and $\nu_{j}^{5} / c \lambda=O\left(|\lambda|^{2 / 3}\right)$, it is clear that for small $\delta_{2}>0$ we may solve for $\beta$ by fixed point iteration, and that $\beta=$ $O\left(\gamma^{2}|\lambda|^{-2 / 3}+|\lambda|^{2 / 3}\right)=o(1)$ as $\delta_{2} \rightarrow 0$. This proves (6.9). The expansion (6.10) is more easily proved using $\left(6.1_{+}\right)$. 
Finally, for large $\lambda$, corresponding to Regime IV, we have the following approximations to the roots.

Proposition 6.5. Suppose $0<\gamma_{0}<1$; then the four roots of $\mathscr{P}(\mu)$ may be labeled so that the following approximations are valid, uniformly for $0 \leq \gamma \leq \gamma_{0}$, as $|\lambda| \rightarrow \infty$ :

$$
\begin{array}{ll}
\mu_{1}=-1+O\left(|\lambda|^{-2}\right), & \mu_{4}=1+O\left(|\lambda|^{-2}\right), \\
\mu_{2}=\lambda-i / c+O\left(|\lambda|^{-2}\right), & \mu_{3}=\lambda+i / c+O\left(|\lambda|^{-2}\right) .
\end{array}
$$

This result was proved for fixed values of $\gamma>0$ in [60], and it is a simple matter to check in the proof that the error terms can be bounded uniformly for $0 \leq \gamma \leq \gamma_{0}$, for any $\gamma_{0} \in(0,1)$.

\section{Eigenvectors and eigenvalues at infinity}

Here we describe explicitly the matrices $A(s, \lambda, \gamma)$ and $A^{\infty}(\lambda, \gamma)$ in (4.1) and (4.2) for each of the Boussinesq eigenvalue problems in (3.1). We also verify that the structural hypotheses $\mathrm{H} 1-\mathrm{H} 3$ hold, which are needed to define the Evans function $D(\lambda, \gamma)$ in Section 4. Furthermore, we describe the left and right eigenvectors of $A^{\infty}$, which are needed in the proofs of Propositions 5.5 and 5.7.

In this section we simplify slightly the notation regarding the solitary-wave profile, writing simply $\eta_{c}$ in place of $\tilde{\eta}_{c}$ and $u_{c}$ in place of $\tilde{u}_{c}$, and $\eta_{c}^{\prime}=\partial_{s} \tilde{\eta}_{c}$, etc. We make considerable use of the following identity for the solitary-wave profiles of (2.1) in cases B2 and B3, which follows from integrating the equations in (2.5) for the steady state:

$$
\left(1-\frac{1}{3} u_{c}\right)\left(1+\frac{1}{3} \eta_{c}\right)=1 .
$$

Corresponding to the definition of the 4-vector $y$ in (4.7), the matrix $A=A(s, \lambda, \gamma)$ derived from (3.1) is given respectively as

$$
A=\left(\begin{array}{cccc}
0 & 1 & 0 & 0 \\
0 & 0 & 1 & 0 \\
0 & 0 & 0 & 1 \\
\lambda^{2}-\eta_{c}^{\prime \prime} & -2 \lambda-2 \eta_{c}^{\prime} & \gamma^{2}-\lambda^{2}-\eta_{c} & 2 \lambda
\end{array}\right)
$$




$$
\begin{gathered}
A=\left(\begin{array}{cccc}
0 & 1 & 0 & 0 \\
0 & 0 & 1 & 0 \\
-\frac{\lambda+(1 / 3) u_{c}^{\prime}}{\left(1+(1 / 3) \eta_{c}\right)^{2}} & \left(1-\frac{1}{3} u_{c}\right)^{3}-1+\gamma^{2}-\lambda^{2} & 2 \lambda & -\left(\lambda+\frac{1}{3} u_{c}^{\prime}\right)-\frac{1}{3} \frac{n_{c}^{\prime}}{\left(1+(1 / 3) \eta_{c}\right)^{2}} \\
-\frac{\lambda+(1 / 3) u_{c}^{\prime}}{1+(1 / 3) \eta_{c}} & \left(1-\frac{1}{3} u_{c}\right)^{2} & 0 & -\frac{1}{3} \eta_{c}^{\prime}\left(1-\frac{1}{3} u_{c}\right)
\end{array}\right), \\
A=\left(\begin{array}{cccc}
0 & 0 & 0 \\
-\left(\lambda+\frac{1}{3} u_{c}^{\prime}\right)-\frac{1}{3} \frac{\eta_{c}^{\prime}\left(1-\gamma^{2}\right)}{1-(1 / 3) u_{c}} & \left(1-\frac{1}{3} u_{c}\right)-\frac{1-\gamma^{2}}{\left(1+(1 / 3) \eta_{c}\right)^{2}} & \lambda & -\left(1-\gamma^{2}\right) \frac{\left(\lambda+(1 / 3) u_{c}^{\prime}\right)}{1-(1 / 3) u_{c}} \\
\frac{1}{3} \eta_{c}^{\prime}\left(1+\frac{1}{3} \eta_{c}\right) & \left(1+\frac{1}{3} \eta_{c}\right)^{2} & 0 & \frac{\lambda+(1 / 3) u_{c}^{\prime}}{1-(1 / 3) u_{c}}
\end{array}\right) .
\end{gathered}
$$

The corresponding matrix $A^{\infty}=A^{\infty}(\lambda, \gamma)$ is

$$
\begin{aligned}
& A^{\infty}=\left(\begin{array}{cccc}
0 & 1 & 0 & 0 \\
0 & 0 & 1 & 0 \\
0 & 0 & 0 & 1 \\
\lambda^{2} & -2 \lambda & \gamma^{2}-\lambda^{2} & 2 \lambda
\end{array}\right),\left(\begin{array}{cccc}
0 & 1 & 0 & 0 \\
0 & 0 & 1 & 0 \\
-\lambda & \gamma^{2}-\lambda^{2} & 2 \lambda & -\lambda \\
-\lambda & 1 & 0 & 0
\end{array}\right), \\
&\left(\begin{array}{cccc}
0 & 1 & 0 & 0 \\
0 & 0 & 1 & 0 \\
-\lambda & \gamma^{2} & \lambda & -\left(1-\gamma^{2}\right) \lambda \\
0 & 1 & 0 & \lambda
\end{array}\right) .
\end{aligned}
$$

In light of the expressions above for $A(s, \lambda, \gamma)$ and $A^{\infty}(\lambda, \gamma)$, the estimates in Corollary 2.2 for $\eta_{c}=\tilde{\eta}_{c}$ and its derivatives, and the relation (7.1), it is easy to deduce the following.

LEMMA 7.1. (i) The matrices $A(s, \lambda, \gamma)$ and $A^{\infty}(\lambda, \gamma)$ are jointly continuous functions of their arguments for $(\lambda, \gamma) \in \mathbb{C} \times[0,1)$, and are analytic in $\lambda$.

(ii) Given any compact set $\Omega_{1} \subset \mathbb{C} \times[0,1)$, there exists a constant $K_{*}$ such that for any $(\lambda, \gamma) \in \Omega_{1}$,

$$
\left|A(s, \lambda, \gamma)-A^{\infty}(\lambda, \gamma)\right| \leq K_{*} \gamma^{2} e^{-|\gamma s|}, \quad-\infty<s<\infty .
$$

From this result, it is evident that the hypotheses H1-H3 (in Section 4) hold on any domain $\Omega^{\gamma}$. Taken together with the results of Section 6 and the treatment in [60], we may conclude that the Evans function is defined as asserted in Proposition 4.4, except that the joint continuity will be proved in Section 8. 
We omit the explicit description of the matrices $A_{*}(\xi, \Lambda, \gamma)$ and $A_{*}^{\infty}(\Lambda, \gamma)$, which correspond to the system (4.14) arising from the eigenvalue problem in the $\mathrm{KdV}$ scaling. It is evident from the structure of Equations (3.3) and the estimates of Corollary 2.2 that they have the following properties, however. From this we deduce that the Evans function $D_{*}(\Lambda, \gamma)$ is defined as asserted in Proposition 4.9 (except for the joint continuity).

LEMMA 7.2. (i) The matrices $A_{*}(\xi, \Lambda, \gamma)$ and $A_{*}^{\infty}(\Lambda, \gamma)$ are jointly continuous functions of their arguments for $(\Lambda, \gamma) \in \mathbb{C} \times[0,1)$ and are analytic functions of $\Lambda$.

(ii) Given any compact set $\Omega_{1} \subset \mathbb{C} \times[0,1)$, there exists a constant $K_{*}$ such that for any $(\Lambda, \gamma) \in \Omega_{1}$,

$$
\left|A_{*}(\xi, \Lambda, \gamma)-A_{*}^{\infty}(\Lambda, \gamma)\right| \leq K_{*} e^{-|\xi|}, \quad-\infty<\xi<\infty .
$$

It is straightforward to compute that for each case B1-B3, the determinant of $A^{\infty}(\lambda, \gamma)-\mu I$ is $\mathscr{P}(\mu ; \lambda, \gamma)$ as defined in (4.8), so the eigenvalues are given by the $\mu_{j}$ discussed in Section 4 . Next, we describe the eigenvectors of $A^{\infty}$, corresponding to eigenvalues that are simple and nonzero; this shall suffice for the analysis we shall perform.

Given a simple eigenvalue $\mu_{j}$, the corresponding right eigenvector, denoted by $\mathbf{v}_{j}$, is given (respectively for cases B1-B3) by

$$
\mathbf{v}_{j}=\left(\begin{array}{c}
1 \\
\mu_{j} \\
\mu_{j}^{2} \\
\mu_{j}^{3}
\end{array}\right), \quad\left(\begin{array}{c}
1 \\
\mu_{j} \\
\mu_{j}^{2} \\
\left(\mu_{j}-\lambda\right) / \mu_{j}
\end{array}\right), \quad\left(\begin{array}{c}
1 \\
\mu_{j} \\
\mu_{j}^{2} \\
\mu_{j} /\left(\mu_{j}-\lambda\right)
\end{array}\right) .
$$

The left eigenvector corresponding to $\mu_{j}$ will be denoted $\mathbf{w}_{j}$, and is taken to be normalized so that $\mathbf{w}_{j} \mathbf{v}_{j}=1$. Then $\mathbf{w}_{i}$ is given (respectively), for $i=1 \ldots 4$, as follows:

$$
\begin{aligned}
\frac{\mathbf{w}_{i}}{\omega_{i}}=\left(\frac{\lambda^{2}}{\mu_{i}}, \frac{\lambda\left(\lambda-2 \mu_{i}\right)}{\mu_{i}^{2}}, \mu_{i}-2 \lambda, 1\right),( & \left.-\frac{\lambda\left(\mu_{i}-\lambda\right)}{\mu_{i}^{2}}, \mu_{i}-2 \lambda, 1,-\frac{\lambda}{\mu_{i}}\right), \\
& \left(-\frac{\lambda}{\mu_{i}}, \mu_{i}-\lambda, 1, \frac{\lambda\left(1-\gamma^{2}\right)}{\lambda-\mu_{i}}\right) .
\end{aligned}
$$


Here the normalizing factors $\omega_{i}$ are given respectively by

$$
\omega_{i}=\frac{\mu_{i}}{2\left(\mu_{i}^{3}-\lambda\right)\left(\mu_{i}-\lambda\right)}, \quad \frac{\mu_{i}^{2}}{2\left(\mu_{i}^{3}-\lambda\right)\left(\mu_{i}-\lambda\right)}, \quad \frac{\mu_{i}}{2\left(\mu_{i}^{3}-\lambda\right)}
$$

\section{Joint continuity of the Evans function}

Here we briefly explain why the Evans function $D(\lambda, \gamma)$ corresponding to the system (4.1) is jointly continuous in $(\lambda, \gamma)$. This will complete the proof of Proposition 4.4. Proposition 4.10 is proved in the same way.

First, we need to recall the precise definition of the Evans function as studied in [60]. (This is necessary for the considerations in Sections 9 and 10 as well.) Recall that for $\lambda \in \Omega^{\gamma}$, the system (4.1) has a unique solution, which is $o\left(e^{\mu * s}\right)$ as $s \rightarrow \infty$, given by $y^{+}(s, \lambda, \gamma)$, with the normalization

$$
y^{+}(s, \lambda, \gamma) \sim e^{\mu_{1} s} \mathbf{v}_{1} \quad \text { as } s \rightarrow+\infty
$$

Associated with (4.1) is the transposed system

$$
\frac{d z}{d s}=-z A(s, \lambda, \gamma)
$$

in which $z$ is regarded as a row vector. Let $\mathbf{w}_{1}$ be a left eigenvector of $-A^{\infty}$ corresponding to the eigenvalue $-\mu_{1}$, normalized by requiring $\mathbf{w}_{1} \cdot \mathbf{v}_{1}=1$. Then (8.2) has a unique solution $z^{-}(s, \lambda, \gamma)$ satisfying

$$
z^{-}(s, \lambda, \gamma) \sim e^{-\mu_{1} s} \mathbf{w}_{1} \quad \text { as } s \rightarrow-\infty .
$$

The definition of the Evans function is

$$
D(\lambda, \gamma)=z^{-}(s, \lambda, \gamma) \cdot y^{+}(s, \lambda, \gamma)
$$

This scalar product is independent of $s$. According to the results of [60], because the hypotheses $\mathrm{H} 1-\mathrm{H} 4$ hold on the domain $\Omega^{\gamma}$ for $0<\gamma \leq \gamma_{0}$, $D(\lambda, \gamma)$ is well defined for $\lambda \in \Omega^{\gamma}$ and is analytic in $\lambda$.

It remains to explain the joint continuity in $(\lambda, \gamma)$. Introduce the notation $\phi=\phi(s, \lambda, \gamma), B=B(\lambda, \gamma)$, and $R=R(s, \lambda, \gamma)$, by requiring

$$
y^{+}(s)=e^{\mu_{1} s}\left(\mathbf{v}_{1}+\phi(s)\right), \quad B=A^{\infty}-\mu_{1} I, \quad R(s)=A(s)-A^{\infty} .
$$


(Here the dependence on $(\lambda, \gamma)$ is suppressed.) Then $\partial_{s} \phi=(B+R)\left(\mathbf{v}_{1}+\phi\right)$ and $B \mathbf{v}_{1}=0$. Following the analysis in Section 1 of [60], we have that

$$
\phi(s)=\mathbf{v}_{1}-\int_{s}^{\infty} e^{(s-r) B} R(r)\left(\mathbf{v}_{1}+\phi(r)\right) d r .
$$

The function $\phi$ is determined from this equation using fixed-point iteration. Now, it is evident from Lemma 7.1 that the quantities $B, R(s)$, and $\mathbf{v}_{1}$ depend continuously on $(\lambda, \gamma)$ in $\Omega$, and that in any compact subset of $\Omega$, we have $\left|e^{s B}\right| \leq C$ for all $s<0$, and $\int_{|s| \geq L}|R(s)| d s \rightarrow 0$ as $L \rightarrow \infty$, uniformly in $(\lambda, \gamma)$. Now, it is straightforward to prove that since $B, R$, and $\mathbf{v}_{1}$ in (8.5) are jointly continuous in $(\lambda, \gamma)$, then so is $\phi(s, \lambda, \gamma)$, for any fixed $s$.

Therefore, $y^{+}(s, \lambda, \gamma)$ is jointly continuous in $(\lambda, \gamma)$ for any fixed $s$. In a similar manner one may prove that $z^{-}(s, \lambda, \gamma)$ is jointly continuous in $(\lambda, \gamma)$ for fixed $s$. Then by the definition (8.4), the Evans function $D(\lambda, \gamma)$ is jointly continuous.

\section{Order of the zero at the origin}

Here we study the behavior of the Evans function at the origin, as promised in Section 5. The value $\lambda=0$ is always an eigenvalue for (3.1), as a consequence of the invariance of (1.1) under spatial translations. A corresponding eigenfunction may be obtained by differentiating the corresponding equation for the solitary wave profile from (2.5). From this we deduce that the Evans function vanishes at the origin. Additionally, we employ an integral formula for derivatives of the Evans function, derived by Pego and Weinstein [60], to deduce the following result, which is needed in the proof of Corollary 5.2. Recall that for $\gamma>0, D(\lambda, \gamma)=D_{*}(\Lambda, \gamma)$.

Proposition 9.1. For $0<\gamma \leq \gamma_{0}$, the value $\lambda=0$ is a zero of order at least two for the Evans function $D(\lambda, \gamma)$. That is,

$$
D(0, \gamma)=\partial_{\lambda} D(0, \gamma)=0 .
$$

Proof: The proof is done for the case B1 in [46]. For cases B2 and B3, we show that $D(0, \gamma)=0$, then use the formula (1.22) of [60] (see also [63]). Using the definitions in Section 8, this formula yields an expression for the derivative of the Evans function at the origin, namely

$$
D^{\prime}(0)=-\int_{-\infty}^{\infty} z^{-}(s, 0) \frac{\partial A}{\partial \lambda}(s, 0) y^{+}(s, 0) d s .
$$


Here and throughout the rest of this section, the $\gamma$ argument is suppressed, and $\gamma$ is regarded as fixed. To prove Proposition 9.1, we identify $y^{+}$and $z^{-}$ and use the characterization (4.5) and the formula (9.1) to show that $D(0)=0$ and $D^{\prime}(0)=0$.

LEMMA 9.2. For each case B2 and B3, there exists a number $\beta \neq 0$ such that $y^{+}(s, 0)=\beta \tilde{y}(s)$, where (respectively):

$$
\tilde{y}=\left(\partial_{s} \eta_{c}, \partial_{s}^{2} \eta_{c}, \partial_{s}^{3} \eta_{c}, \partial_{s} u_{c}\right) \quad \text { or } \quad\left(\partial_{s} u_{c}, \partial_{s}^{2} u_{c}, \partial_{s}^{3} u_{c}, \partial_{s} \eta_{c}\right)
$$

Proof: Since $\left(\eta_{c}, u_{c}\right)$ are stationary solutions of (2.1), by differentiating with respect to $s$, it follows that $(h, v)=\left(\partial_{s} \eta_{c}, \partial_{s} u_{c}\right)$ satisfies (3.1) with $\lambda=0$. If $\tilde{y}(s)$ denotes the right-hand side of (9.2), then from (4.2) it follows that $\tilde{y}$ satisfies (4.1) with $\lambda=0$. Now the lemma follows, because $\tilde{y}(s)$ and $y^{+}(s, 0)$ both decay exponentially as $s \rightarrow+\infty$.

To determine $z^{-}$, it is useful to write the system $y^{\prime}=A y$ in an equivalent form when $\lambda=0$, namely as

$$
\left(B_{1}(s) y\right)^{\prime}=B_{2} y, \quad \text { where } B_{1}^{-1}\left(B_{2}-B_{1}^{\prime}\right)=A(s, 0) .
$$

The matrices $B_{1}(s)$ and $B_{2}$ may be chosen as follows, in cases B2 and B3 respectively. ( $B_{2}$ is the same in both cases. Compare (3.1).)

$$
\begin{aligned}
& B_{1}(s)=\left(\begin{array}{cccc}
1 & 0 & 0 & 0 \\
0 & 1 & 0 & 0 \\
1-\gamma^{2} & 0 & 1 & -\left(1-\frac{1}{3} u_{c}\right) \\
-\left(1-\frac{1}{3} u_{c}\right) & 0 & 0 & 1+\frac{1}{3} \eta_{c}
\end{array}\right) \quad \text { or } \\
& \left(\begin{array}{cccc}
1 & 0 & 0 & 0 \\
0 & 1 & 0 & 0 \\
-\left(1-\frac{1}{3} u_{c}\right) & 0 & 1 & 1-\gamma^{2} \\
1+\frac{1}{3} \eta_{c} & 0 & 0 & -\left(1-\frac{1}{3} u_{c}\right)
\end{array}\right) \\
& B_{2}=\left(\begin{array}{llll}
0 & 1 & 0 & 0 \\
0 & 0 & 1 & 0 \\
0 & 0 & 0 & 0 \\
0 & 0 & 0 & 0
\end{array}\right)
\end{aligned}
$$

To find $z$ satisfying $z^{\prime}=-z A$, it suffices to find $\tilde{z}$ so that

$$
\tilde{z}^{\prime} B_{1}=-\tilde{z} B_{2}
$$

and then let $z=\tilde{z} B_{1}$. 
LEMMA 9.3. For each case B2 and B3, there exists a number $\beta$ such that $z^{-}(s, 0)=\beta \tilde{z}(s) B_{1}(s)$, where respectively:

$$
\begin{aligned}
\tilde{z}(s)= & \left(\partial_{s}^{2} \eta_{c},-\partial_{s} \eta_{c}, \eta_{c}, u_{c}\right) \quad \text { or } \\
& \left(\partial_{s}^{2} u_{c},-\partial_{s} u_{c}, u_{c}, 3\left(1-\gamma^{2}\right) \log \left(1+\frac{1}{3} \eta_{c}\right)\right) .
\end{aligned}
$$

Proof: Because $(h, v)=\left(\partial_{s} \eta_{c}, \partial_{s} u_{c}\right)$ satisfies (3.1) for $\lambda=0$, it is easy to verify that $\tilde{z}(s)$ defined as above satisfies (9.4) in each case. Since $\tilde{z}(s) B_{1}(s)$ $\rightarrow 0$ as $s \rightarrow-\infty$, it follows that $\tilde{z}$ is a scalar multiple of $z^{-}$.

Proof of Proposition 9.1: From Lemma 9.2, it is clear that $y^{+}(s, 0) \rightarrow 0$ as $s \rightarrow-\infty$. By the characterization of the Evans function in (4.5), it follows $D(0)=0$, since $\mu_{1}(0)<0$.

We use the formula (9.1) to compute $D^{\prime}(0)$. For each case B2 and B3, respectively, we compute

$$
\begin{aligned}
& \partial_{\lambda} A(s, 0)=\left(\begin{array}{cccr}
0 & 0 & 0 & 0 \\
0 & 0 & 0 & 0 \\
-\left(1-\frac{1}{3} u_{c}\right)^{2} & 0 & 2 & -1 \\
-\left(1-\frac{1}{3} u_{c}\right) & 0 & 0 & 0
\end{array}\right) \text { or } \\
&\left(\begin{array}{rrrc}
0 & 0 & 0 & 0 \\
0 & 0 & 0 & 0 \\
-1 & 0 & 1 & -\left(1-\gamma^{2}\right)\left(1+\frac{1}{3} \eta_{c}\right) \\
0 & 0 & 0 & 1+\frac{1}{3} \eta_{c}
\end{array}\right) .
\end{aligned}
$$

To show that $D^{\prime}(0)=0$ it suffices to integrate the expression $\tilde{z} B_{1} \partial_{\lambda} A \tilde{y}$, from Lemmas 9.2 and 9.3. We find that this expression equals, for cases B2 and $\mathrm{B} 3$, respectively,

$$
\tilde{z} B_{1} \partial_{\lambda} A \tilde{y}=-\left(1-\frac{1}{3} u_{c}\right)^{2} \eta_{c} \partial_{s} \eta_{c}+2 \eta_{c} \partial_{s}^{3} \eta_{c}-\eta_{c} \partial_{s} u_{c}-u_{c}\left(1-\frac{1}{3} u_{c}\right) \partial_{s} \eta_{c}
$$

or

$$
-u_{c} \partial_{s} u_{c}+u_{c} \partial_{s}^{3} u_{c}-3\left(1-\gamma^{2}\right) \log \left(1+\frac{1}{3} \eta_{c}\right) \partial_{s} \eta_{c}
$$

Since $u_{c}$ can be replaced by $\eta_{c}$ and vice versa using the relation $\left(1-\frac{1}{3} u_{c}\right)(1+$ $\left.\frac{1}{3} \eta_{c}\right)=1$, every term in these expressions is a perfect derivative, so it follows that

$$
\int_{-\infty}^{\infty} \tilde{z}(s) B_{1}(s) \partial_{\lambda} A(s, 0) \tilde{y}(s) d s=0
$$

Therefore $D^{\prime}(0)=0$. 


\section{Large values of the eigenvalue parameter}

In this section we complete the proof of Theorem 5.1, by proving Propositions 5.5 and 5.7. For this, we need to obtain appropriate estimates on $|D(\lambda, \gamma)-1|$ in Regimes II and IV. In all cases but one, the necessary estimates are obtained from the following lemma.

LEMMA 10.1. Consider the $n \times n$ system

$$
\frac{d v}{d s}=B v(s)+F(s)\left(e_{1}+v(s)\right), \quad 0 \leq s<\infty .
$$

Here $e_{1}=(1,0, \ldots, 0)^{t}$. Assume that $B$ is a diagonal matrix with $B_{11}=0$ and $\Re B_{j j} \geq 0$ for $j>1$, and that the matrix $F(s)$ is continuous in $s$ with $\lim _{s \rightarrow \infty} F(s)=0$ and $\int_{0}^{\infty}|F(s)| d s<\infty$. Then there exist positive constants $\delta_{0}$ and $C_{0}$, such that if $\int_{0}^{\infty}|F(s)| d s<\delta_{0}$, then (10.1) has a solution $v(s)$ with $\lim _{s \rightarrow \infty} v(s)=0$, and this solution satisfies

$$
\sup _{0 \leq s<\infty}|v(s)| \leq C_{0} \int_{0}^{\infty}|F(s)| d s
$$

Proof: The solution $v(s)$ is obtained by fixed-point iteration using the equation

$$
v(s)=(\mathscr{T} v)(s) \equiv-\int_{s}^{\infty} e^{(s-r) B} F(r)\left(e_{1}+v(r)\right) d r
$$

along with the estimate $\left|e^{B s}\right| \leq C$ for $s \leq 0$, and the estimate

$$
|(\mathscr{T} v)(s)| \leq C \int_{s}^{\infty}|F(r)| d r\left(1+\sup _{0 \leq r<\infty}|v(r)|\right) .
$$

From (10.3) and (10.4) it is easy to establish the bound (10.2)

To obtain a bound on $|D(\lambda, \gamma)-1|$, we use Lemma 10.1, with the matrices $B$ and $F$ constructed as follows. $B$ is obtained by diagonalizing $A^{\infty}-\mu_{1} I$. Because of Propositions 6.4 and 6.5, the eigenvalues of $A_{\infty}$ are distinct in Regimes II and IV. Let $V$ be the matrix containing the right eigenvector $\mathbf{v}_{j}$ from (7.4) in column $j$, and let $W$ be the matrix containing the left eigenvector $\mathbf{w}_{i}$ from (7.5) in row $i$. Then $W V=I$. Suppressing the dependence on $(\lambda, \gamma)$, we set

$$
\begin{gathered}
B=W\left(A^{\infty}-\mu_{1} I\right) V=\operatorname{diag}\left\{\mu_{j}-\mu_{1}\right\}, \\
F(s)=W R(s) V=W\left(A(s)-A^{\infty}\right) V .
\end{gathered}
$$


Recall the characterization of $y^{+}(s)$ in (4.6), and define $v^{+}(s)$ from

$$
y^{+}(s)=e^{\mu_{1} s} V\left(e_{1}+v^{+}(s)\right) \text {. }
$$

Then $v=v^{+}$satisfies (10.1) and $v^{+}(s) \rightarrow 0$ as $s \rightarrow \infty$. In similar fashion we define $w^{-}(s)$ from $z^{-}(s)$ using $z^{-}(s)=\left(w^{-}(s)+e_{1}^{t}\right) W e^{-\mu_{1} s}$. Then $w=w^{-}$ satisfies

$$
\frac{d w}{d s}=-w(s) B-\left(w(s)+e_{1}^{t}\right) F(s), \quad-\infty<s \leq 0
$$

and $w^{-}(s) \rightarrow 0$ as $s \rightarrow-\infty$. Taking the transpose and reflecting in $s$, we may conclude that if $\int_{-\infty}^{\infty}|F(s)| d s<\delta_{0}$, from Lemma 10.1 we obtain the estimate

$$
\sup _{0 \leq s<\infty}\left|v^{+}(s)\right|+\sup _{-\infty<s \leq 0}\left|w^{-}(s)\right| \leq C \int_{-\infty}^{\infty}|F(s)| d s .
$$

Therefore, since $D(\lambda, \gamma)=z^{-} \cdot y^{+}=\left(e_{1}^{t}+w^{-}\right)\left(e_{1}+v^{+}\right)$, we have:

COROLlary 10.2. Consider the system (4.1) under the hypotheses of Section 4 and assume $A^{\infty}(\lambda)$ is diagonalizable with $V$ and $W$ being the matrices of right (respectively, left) eigenvectors. Let $F(s)=W\left(A(s)-A^{\infty}\right) V$. There exists a number $\delta_{0}>0$ such that if $\int_{-\infty}^{\infty}|F(s)| d s \leq \delta_{0}$, then

$$
|D(\lambda, \gamma)-1| \leq C \int_{-\infty}^{\infty}|F(s)| d s
$$

This estimate is used to prove Propositions 5.5 and 5.7, except in one case, that of $B 3$ in Regime IV, which requires special treatment.

Because of the structure of the matrices $A$ in (7.1) and $A^{\infty}$ in (7.2), and the estimates for $\tilde{\eta}_{c}$ and $\tilde{u}_{c}$ from Corollary 2.2, it is evident that for $0 \leq \gamma \leq \gamma_{0}, \lambda \in \mathbb{C}$, we have the following estimates on the deviator $R(s, \lambda, \gamma)=A(s, \lambda, \gamma)-A^{\infty}(\lambda, \gamma)$ :

$$
\left|R_{i j}(s, \lambda, \gamma)\right| \leq C \gamma^{2} e^{-|\gamma s|} E_{i j}
$$

where for each case B1, B2, B3, respectively,

$$
E=\left(\begin{array}{cccc}
0 & 0 & 0 & 0 \\
0 & 0 & 0 & 0 \\
0 & 0 & 0 & 0 \\
\gamma^{2} & \gamma & 1 & 0
\end{array}\right),\left(\begin{array}{cccc}
0 & 0 & 0 & 0 \\
0 & 0 & 0 & 0 \\
\gamma+|\lambda| & 1 & 0 & \gamma \\
\gamma+|\lambda| & 1 & 0 & \gamma
\end{array}\right),\left(\begin{array}{cccc}
0 & 0 & 0 & 0 \\
0 & 0 & 0 & 0 \\
\gamma & 1 & 0 & \gamma+|\lambda| \\
\gamma & 1 & 0 & \gamma+|\lambda|
\end{array}\right)
$$


Using the expressions for the entries of $V$ and $W$ in (7.4) and (7.5), it follows that

$$
\left|F_{i j}(s)\right| \leq C \gamma^{2} e^{-|\gamma s|} G_{i j}
$$

where in terms of the roots $\mu_{j}$ we have respectively

$$
\begin{aligned}
G_{i j} & =\frac{\left|\mu_{i}\right|}{\left|\mu_{i}^{3}-\lambda\right|\left|\mu_{i}-\lambda\right|}\left(\gamma^{2}+\gamma\left|\mu_{j}\right|+\left|\mu_{j}\right|^{2}\right), \\
G_{i j} & =\frac{\left|\mu_{i}\right|^{2}}{\left|\mu_{i}^{3}-\lambda\right|\left|\mu_{i}-\lambda\right|}\left(1+\left|\frac{\lambda}{\mu_{i}}\right|\right)\left(\gamma+|\lambda|+\left|\mu_{j}\right|+\gamma\left|\frac{\mu_{j}-\lambda}{\mu_{j}}\right|\right), \\
G_{i j} & =\left|\frac{\mu_{i}}{\mu_{i}^{3}-\lambda}\right|\left(1+\left|\frac{\lambda}{\lambda-\mu_{i}}\right|\right)\left(\gamma+\left|\mu_{j}\right|+(\gamma+|\lambda|)\left|\frac{\mu_{j}}{\mu_{j}-\lambda}\right|\right) .
\end{aligned}
$$

Proof of Proposition 5.5: Using Proposition 6.4, there exists $\gamma_{2}>0$ such that for $0<\gamma<\gamma_{2}$, the following estimates hold uniformly for $\lambda \in S_{2}$ as $\delta \rightarrow 0$ :

$$
\begin{array}{rlrl}
\text { for } j=1,2,3: & \multicolumn{1}{c}{\text { for } j=4:} \\
\left|\mu_{j}\right|=2 c|\lambda|^{1 / 3}(1+o(1)), & & \left|\frac{c \lambda}{c+1}\right|(1+o(1)), \\
\left|\mu_{j}-\lambda\right|=2 c|\lambda|^{1 / 3}(1+o(1)), & \left|\frac{\lambda}{c+1}\right|(1+o(1)), \\
\left|\mu_{j}^{3}-\lambda\right|=|\lambda|(1+2 c)(1+o(1)), & |\lambda|(1+o(1))
\end{array}
$$

Using these estimates, we find that since $\gamma \leq \delta^{2 / 3}$ in Regime II when $S_{2}$ is nonempty, for each case B1, B2, and B3 we have

$$
\gamma G_{i j} \leq C \delta^{1 / 3}
$$

Since $\int_{-\infty}^{\infty} \gamma e^{-|\gamma s|} d s=2$, from these estimates and (10.8) and (10.6) we find that

$$
|D(\lambda, \gamma)-1| \leq C \delta^{1 / 3}
$$

This proves Proposition 5.5. 
Proof of Proposition 5.7 for cases B1 and B2: According to Proposition 6.5, for $0<\gamma \leq \gamma_{0}$ and $\lambda \in S_{4}$, the following estimates hold uniformly as $\delta \rightarrow 0$ :

$$
\begin{aligned}
\text { for } j=1,4: & & \text { for } j=A 2,3: \\
\left|\mu_{j}\right|=1+o(1), & & |\lambda|(1+o(1)), \\
\left|\mu_{j}-\lambda\right|=|\lambda|(1+o(1)), & & c^{-1}+o(1), \\
\left|\mu_{j}^{3}-\lambda\right|=|\lambda|(1+o(1)), & & |\lambda|^{3}(1+o(1)) .
\end{aligned}
$$

Using these estimates in (10.9), it is straightforward to verify that for all $i, j$ we have that for some constant $C$, if $\delta$ is sufficiently small, then

$$
\gamma G_{i j} \leq C \gamma
$$

As previously, this implies that

$$
|D(\lambda, \gamma)-1| \leq C \gamma
$$

This completes the Proof of Proposition 5.7 for the cases B1 and B2.

Proof of Proposition 5.7 in the case B3: In the case B3, corresponding to (10.13), it appears that we can obtain only from (10.9.B3), the bound $G_{i j} \leq C|\lambda|$ when $j=2,3$, which is insufficient to prove Proposition 5.7, because in this case $\int_{-\infty}^{\infty}|F(s)| d s=O(\lambda \gamma)$ does not tend to zero as $\gamma \rightarrow 0$ uniformly for $\lambda \in S_{4}$.

A more delicate approach is necessary. Roughly speaking, instead of using Lemma 10.1, we work with a variant, with $B$ in (10.1) replaced by adding to it a variable coefficient symmetric matrix, which accounts for the $O(\lambda \gamma)$ terms in $F$.

Toward this end, we write $R=A-A^{\infty}=R^{(1)}+R^{(2)}, F=F^{(1)}+F^{(2)}=$ $W R^{(1)} V+W R^{(2)} V$, where

$$
R^{(1)}=\frac{1}{3} \eta_{c} \lambda\left(\begin{array}{cccc}
0 & 0 & 0 & 0 \\
0 & 0 & 0 & 0 \\
0 & 0 & 0 & -\left(1-\gamma^{2}\right) \\
0 & 0 & 0 & 1
\end{array}\right)
$$


and note that $\left|R_{i j}^{(2)}\right| \leq C \gamma^{2} e^{-|\gamma s|} E_{i j}^{(2)}$, where

$$
E^{(2)}=\left(\begin{array}{llll}
0 & 0 & 0 & 0 \\
0 & 0 & 0 & 0 \\
\gamma & 1 & 0 & \gamma \\
\gamma & 1 & 0 & \gamma
\end{array}\right)
$$

The part $R^{(2)}$ is estimated as in the cases B1 and B2; we find that

$$
\left|F_{i j}^{(2)}\right| \equiv\left|\mathbf{w}_{i} R^{(2)} \mathbf{v}_{j}\right| \leq C \gamma^{2} e^{-|\gamma s|} G_{i j}^{(2)}
$$

where

$$
G_{i j}^{(2)}=\left|\frac{\mu_{i}}{\mu_{i}^{3}-\lambda}\right|\left(1+\left|\frac{\lambda}{\lambda-\mu_{i}}\right|\right)\left(\gamma+\left|\mu_{j}\right|+\gamma\left|\frac{\mu_{j}}{\mu_{j}-\lambda}\right|\right) .
$$

Using the estimates in (10.12), it follows that $\left|G_{i j}^{(2)}\right| \leq C$, uniformly for $0<\gamma \leq \gamma_{0}$ and $|\lambda| \geq \delta^{-1}$, for $\delta$ sufficiently small.

Next, we compute that

$$
\left|F_{i j}^{(1)}\right| \equiv \mathbf{w}_{i} R^{(1)} \mathbf{v}_{j}=-\frac{1}{6} \eta_{c} \lambda\left(1-\gamma^{2}\right) \frac{\mu_{i}}{\mu_{i}^{3}-\lambda} \frac{\mu_{i}}{\mu_{i}-\lambda} \frac{\mu_{j}}{\mu_{j}-\lambda} .
$$

Using the expressions in Proposition 6.5, one may calculate that, for $\lambda \in S_{4}$ sufficiently large,

$$
\begin{aligned}
F_{i j}^{(1)}(s) & =\mathbf{w}_{i} R^{(1)}(s) \mathbf{v}_{j} \\
& =\frac{1}{6} \eta_{c}(s) \cdot \begin{cases}O(1) & \text { if either } i \text { or } j \text { is } 1 \text { or } 4, \\
\lambda(-1)^{i+j}+O(1) & \text { if both } i \text { and } j \text { are } 2 \text { or } 3 .\end{cases}
\end{aligned}
$$

Now define

$$
\begin{aligned}
\tilde{B}(s)= & \left(\begin{array}{cccc}
0 & 0 & 0 & 0 \\
0 & \mu_{2}-\mu_{1} & 0 & 0 \\
0 & 0 & \mu_{3}-\mu_{1} & 0 \\
0 & 0 & 0 & \mu_{4}-\mu_{1}
\end{array}\right) \\
& +\frac{1}{6} \eta_{c}(s) \lambda\left(\begin{array}{rrrr}
0 & 0 & 0 & 0 \\
0 & 1 & -1 & 0 \\
0 & -1 & 1 & 0 \\
0 & 0 & 0 & 0
\end{array}\right)
\end{aligned}
$$


Then we have that

$$
W\left(A-\mu_{1} I\right) V=B+F(s)=\tilde{B}(s)+\tilde{F}(s),
$$

where

$$
|\tilde{F}(s)| \leq C \gamma^{2} e^{-|\gamma s|}
$$

for $\lambda \in S_{4}$ if $\delta$ is sufficiently small.

Note that the equation for $v^{+}$(recall $y^{+}=e^{\mu_{1} s} V\left(e_{1}+v^{+}\right)$) can be written as

$$
\frac{d v^{+}}{d s}=\tilde{B}(s) v^{+}+\tilde{F}\left(e_{1}+v^{+}\right)
$$

This is as in (10.1) except that $B$ is replaced by $\tilde{B}(s)$. The nonconstant part of $\tilde{B}(s)$ is of the form $\lambda$ times a positive definite matrix. This structure now is exploited to give a bound on the fundamental matrix for the system:

$$
\frac{d \alpha}{d s}=\tilde{B}(s) \alpha
$$

LEMMA 10.3. If $\tilde{B}(s)$ is given by (10.15) and if $X(s, \tau)$ is the fundamental matrix of (10.18), satisfying $X(\tau, \tau)=I$, then if $\gamma$ and $\delta$ are sufficiently small, for all $\lambda \in S_{4}$ we have

$$
|X(s, \tau) \rho| \leq|\rho|, \quad \text { for all } s \leq \tau, \rho \in \mathbb{C}^{4}
$$

For case B3 this bound plays a role analogous to the bound $\left|e^{B s}\right| \leq C$ for $s \leq 0$ in the cases B1 and B2. In fact, with the bound (10.19) in hand, the construction and estimation of $v^{+}\left(y^{+}\right)$and $w^{-}\left(z^{-}\right)$go through, using (10.3) with $F(s)$ replaced by $\tilde{F}(s)$ and $e^{(s-r) B}$ replaced by $X(s, r)$. We then have the analogue of (10.6), namely

$$
|D(\lambda, \gamma)-1| \leq C \int_{-\infty}^{\infty}|\tilde{F}(s)| d s
$$

Use of the estimate (10.16) in (10.20) completes the proof for the case B3.

It remains then to prove Lemma 10.3. Let $\alpha(s)=X(s, \tau) \rho$. First note that one can solve explicitly for the first and fourth components of $\alpha(s)$ to get

$$
\left|\alpha_{1}(s)\right|=\left|\rho_{1}\right|, \quad\left|\alpha_{4}(s)\right|=\left|e^{\left(\mu_{4}-\mu_{1}\right)(s-\tau)} \rho_{4}\right| \leq\left|\rho_{4}\right|
$$


To estimate $\mathbf{a} \equiv\left(\alpha_{2}, \alpha_{3}\right)^{t}$, we note that

$$
\frac{d \mathbf{a}}{d s}=\left(\begin{array}{cc}
\mu_{2}-\mu_{1} & 0 \\
0 & \mu_{3}-\mu_{1}
\end{array}\right) \mathbf{a}+\lambda S \mathbf{a}
$$

where

$$
S=\frac{1}{6} \eta_{c}(s)\left(\begin{array}{rr}
1 & -1 \\
-1 & 1
\end{array}\right)
$$

With the notation $\langle\boldsymbol{\alpha}, \boldsymbol{\beta}\rangle=\sum_{i} \alpha_{i} \bar{\beta}_{i}$ for the inner product on $\mathbb{C}^{2}$, we have

$$
\frac{1}{2} \frac{d}{d s}\langle\mathbf{a}(s), \mathbf{a}(s)\rangle \geq \min _{j=2,3} \Re\left(\mu_{j}-\mu_{1}\right)\langle\mathbf{a}(s), \mathbf{a}(s)\rangle+(\Re \lambda)\langle\mathbf{a}(s), S \mathbf{a}(s)\rangle .
$$

Since $S$ is a nonnegative matrix, and $|S(s)| \leq C \gamma^{2}$, with $\mu_{*}(\lambda, \gamma)=$ $\min _{j>1} \mathfrak{R}\left(\mu_{j}-\mu_{1}\right)$, we evidently have

$$
\frac{1}{2} \frac{d}{d s}\langle\mathbf{a}(s), \mathbf{a}(s)\rangle \geq \begin{cases}0 & \text { for } \Re \lambda \geq 0 \\ \left(\mu_{*}-\varepsilon_{0} C \gamma^{2}\right)|\mathbf{a}(s)|^{2} & \text { for }-\varepsilon_{0}<\Re \lambda<0 .\end{cases}
$$

From Proposition 6.5 we deduce that $\mu_{*}=\Re \lambda+1+o(1)$ as $|\lambda| \rightarrow \infty$ with $-\varepsilon_{0}<\Re \lambda$. Therefore if $\gamma$ and $\delta$ are sufficiently small, we conclude that $|\mathbf{a}(s)|$ is nondecreasing in $s$, which completes the proof of the lemma.

\section{Convective linear stability}

Using the results obtained so far, concerning the absence of eigenvalues with respect to the weighted norm in (1.3), we establish a convective linear stability result as described in the introduction, of the type used in proving the asymptotic stability of solitary waves in generalized KdV equations [17] and in the RLW equation [28, 29].

To proceed, one writes the linearized evolution equation from (3.1) in an abstract form

$$
\partial_{\tau} z=\mathscr{A} z
$$

for $z=(h, v)$ on a Hilbert space such as $X_{a}=L_{a}^{2} \times L_{a}^{2}$, where $L_{a}^{2}$ is defined using the weighted $L^{2}$ norm in (1.3). One then shows the following, for sufficiently small $\gamma$ and a suitable value of $a$ :

$\mathrm{R} 1$ The initial value problem for (11.1) is well posed in that $\mathscr{A}$ is the generator of a $C_{0}$ semigroup on $X_{a}$. 
R2 Zero is an isolated eigenvalue of $\mathscr{A}$ of algebraic multiplicity two.

R3 The resolvent $(\lambda-\mathscr{A})^{-1}$ is uniformly bounded in the right half-plane $\Re \lambda>0$, outside any neighborhood of the origin.

It is a direct consequence of a result given by Prüss [26, Corollary 4], applied to the semigroup $\exp (\mathscr{A} t)$ restricted to the spectral complement of the generalized kernel of $\mathscr{A}$, that these facts yield the following convective linear stability result for solitary waves.

THEOREM 11.1. Consider (11.1) in the cases B1 and B3. If $\gamma>0$ is sufficiently small, there exists $a>0$ (of order $O(\gamma)$ ) and positive constants $C$ and $b$ with the following property. Given initial data $z(0)=z_{0} \in X_{a}$ whose spectral projection onto the generalized kernel of $\mathscr{A}$ vanishes, we have

$$
\|z(\tau)\|_{a} \leq C e^{-b \tau}\left\|z_{0}\right\|_{a}, \quad \tau>0
$$

The projection onto the two-dimensional generalized kernel corresponds to infinitesimal shifts in phase and wave speed (or amplitude) among the two-parameter family of solitary waves. So the result in this theorem corresponds to the linearized version of an orbital asymptotic stability result for solitary waves as described in (1.2).

It remains to establish the properties R1-R3 for each case B1 and B3. We omit case B2 because of complications in formulating a well-posed initial value problem in this case.

\subsection{Convective linear stability for the case $\mathrm{B} 1$}

The linear evolution equation for $h(s, t)$ from (3.1.B1) is second order in time and corresponds to the first-order system

$$
\left(\partial_{\tau}-\partial_{s}\right) h+S v=0, \quad S\left(1-\gamma^{2}+\tilde{\eta}_{c}\right) h+\left(\partial_{\tau}-\partial_{s}\right) v=0
$$

where $S=\partial_{s}\left(I-\partial_{s}^{2}\right)^{-1 / 2}$ is an operator of order zero. With $z=(h, v)$, the operator in (11.1) is given by $\mathscr{A}=\mathscr{A}_{*}+\mathscr{B}_{*}$, where

$$
\mathscr{A}_{*}=\left(\begin{array}{cc}
\partial_{s} & -S \\
-S\left(1-\gamma^{2}\right) & \partial_{s}
\end{array}\right), \quad \mathscr{B}_{*}=\left(\begin{array}{cc}
0 & 0 \\
-S \tilde{\eta}_{c} & 0
\end{array}\right) \text {. }
$$

Studying the resolvent of $\mathscr{A}$ on $X_{a}=L_{a}^{2} \times L_{a}^{2}$ is equivalent to studying the resolvent of $\mathscr{A}_{a}=e^{a s} \mathscr{A} e^{-a s}$ on $X_{0}=L^{2} \times L^{2}$, since the map $z \mapsto e^{a s} z$ is an isometry of $X_{a}$ onto $X_{0} . \mathscr{A}_{a}$ may be obtained by replacing $\partial_{s}$ by $\partial_{s}-a$ in the definitions of $\mathscr{A}$ and $S . \mathscr{A}_{a}$ is a bounded perturbation of the operator $\partial_{s}$ with domain $H^{1} \times H^{1}$; therefore $\mathscr{A}_{a}$ is the generator of a $C_{0}$ semigroup on $X_{0}$. This implies that $\mathrm{R} 1$ is true. 
We study the resolvent of $\mathscr{A}_{a}$ using perturbation theory and the Fourier transform. The resolvent of the constant coefficient operator $\mathscr{A}_{*_{a}}=e^{a s} \mathscr{A}_{*} e^{-a s}$ is given by the following formula. If $z=\left(\lambda-\mathscr{A}_{*}\right)^{-1} \zeta$ with $\zeta \in X_{0}$, then $\hat{z}(\xi)=\mathscr{R}(i \xi-a, \lambda) \hat{\zeta}(\xi)$, where

$$
\mathscr{R}(\mu, \lambda)=\frac{1}{\left(\lambda-\mathscr{P}_{+}(\mu)\right)\left(\lambda-\mathscr{P}_{-}(\mu)\right)}\left(\begin{array}{cc}
\lambda-\mu & \hat{S}(\mu) \\
\hat{S}(\mu)\left(1-\gamma^{2}\right) & \lambda-\mu
\end{array}\right)
$$

with $\hat{S}(\mu)=\mu / \sqrt{1-\mu^{2}}$. Here $\mathscr{P}_{ \pm}(\mu)$ are as in $\left(6.1_{ \pm}\right)$, with the dependence on $\gamma$ suppressed.

The norm of the resolvent of $\mathscr{A}_{* a}$ is $\left\|\left(\lambda-\mathscr{A}_{* a}\right)^{-1}\right\|=\sup _{\xi}|\mathscr{R}(i \xi-a, \lambda)|$. From $\left(6.1_{ \pm}\right)$it is clear that this is finite if and only if $\lambda \neq \mathscr{P}_{ \pm}(i \xi-a)$ for all real $\xi$. For $0<a<1$ the proof of Lemma 6.1 implies that with $\mu=i \xi-a$,

$$
\mathfrak{R} \mathscr{P}_{+}(\mu)<\mathfrak{R} \mu=-a<\mathfrak{R} \mathscr{P}_{-}(\mu)
$$

Moreover, Lemma 6.3 implies that for $0<a<-\mu_{m}=\left(1-c^{-2 / 3}\right)^{1 / 2} \approx \gamma / \sqrt{3}$,

$$
\mathfrak{R} \mathscr{P}_{-}(i \xi-a) \leq \mathscr{P}_{-}(-a)<0
$$

for all $\xi$. Therefore, for such $a$, the half-plane $\mathfrak{R} \lambda>\mathscr{P}_{-}(-a)$ lies in the resolvent set of $\mathscr{A}_{*}$. Moreover, it is not hard to show that $\left\|\left(\lambda-\mathscr{A}_{*}\right)^{-1}\right\|$ is uniformly bounded for $\mathfrak{R} \lambda>0$, since then $\left|\lambda-\mathscr{P}_{ \pm}(i \xi-a)\right| \geq\left|\mathscr{P}_{-}(-a)\right|>0$ uniformly for $\xi$ real.

Henceforth, we restrict our attention to values of $a$ in $\left(0,-\mu_{m}\right)$. Now, we claim that the operator $\left(\lambda-\mathscr{A}_{*_{a}}\right)^{-1}\left(\mathscr{A}_{a}-\mathscr{A}_{*_{a}}\right)$ is compact, which implies that the spectrum of $\mathscr{A}_{a}$ in the half-plane $\mathfrak{R}_{\lambda}>\mathscr{P}_{-}(-a)$ is discrete, consisting only of isolated eigenvalues of finite multiplicity (cf. [64]). We compute that

$$
\left(\lambda-\mathscr{A}_{* a}\right)^{-1}\left(\mathscr{A}_{a}-\mathscr{A}_{* a}\right)=\mathscr{Q} \tilde{\eta}_{c}
$$

where $\mathscr{Q}$ is the pseudodifferential operator with symbol

$$
\hat{\mathscr{Q}}(\mu)=\frac{1}{\left(\lambda-\mathscr{P}_{+}(\mu)\right)\left(\lambda-\mathscr{P}_{-}(\mu)\right)}\left(\begin{array}{cc}
-\hat{S}(\mu)^{2} & 0 \\
-(\lambda-\mu) \hat{S}(\mu) & 0
\end{array}\right),
$$

where $\mu=i \xi-a$. We have that $\lim _{|\xi| \rightarrow \infty} \hat{\mathscr{Q}}(i \xi-a)=0$ and $\lim _{|s| \rightarrow \infty} \tilde{\eta}_{c}(s)=0$ and that these functions are continuous. This immediately implies that $\mathscr{Q} \tilde{\eta}_{c}$ is compact; see [65] for a short proof.

It is not hard to show that if $\lambda$ is an eigenvalue of $\mathscr{A}_{a}$ in the half-plane $\Re \lambda>\mathscr{P}_{-}(-a)$, then the eigenvalue equation in (3.1.B1) has a nontrivial 
solution, which is $o\left(e^{\mu_{1} s}\right)$ as $s \rightarrow-\infty$ and is $o(1)$ as $s \rightarrow+\infty$, and therefore $\lambda$ is a zero of the Evans function $D(\lambda, \gamma)$. Therefore, as a direct consequence of Corollary 5.2, if $\gamma>0$ is sufficiently small (depending on $\sigma$ in (5.1)), then $\mathscr{A}_{a}$ has only one eigenvalue in this half-plane, namely zero.

Consider the multiplicity of zero as an eigenvalue of $\mathscr{A}$ on $X_{a}$. Recall that the solitary wave profile $\tilde{\eta}_{c}$ satisfies

$$
-\left(1-\partial_{s}^{2}\right) \tilde{\eta}_{c}+\left(1-\gamma^{2}\right) \tilde{\eta}_{c}+\frac{1}{2} \tilde{\eta}_{c}^{2}=0 .
$$

Hence if the operator $L=-\left(1-\partial_{s}^{2}\right)+\left(1-\gamma^{2}\right)+\tilde{\eta}_{c}$, we have

$$
L \partial_{s} \tilde{\eta}_{c}=0, \quad L \partial_{\gamma} \tilde{\eta}_{c}=2 \gamma \tilde{\eta}_{c}, \quad L \tilde{\eta}_{c}=\frac{1}{2} \tilde{\eta}_{c}^{2} .
$$

It is convenient to define

$$
\begin{aligned}
\chi & =\left(1-\gamma^{2}\right) \tilde{\eta}_{c}+\frac{1}{2} \tilde{\eta}_{c}^{2}=\left(1-\partial_{s}^{2}\right) \tilde{\eta}_{c}, \\
h_{1} & =\left(1-\gamma^{2}\right)^{-2} \partial_{\gamma}\left(\left(1-\gamma^{2}\right)^{-1} \tilde{\eta}_{c}\right)=2 \gamma \tilde{\eta}_{c}+\left(1-\gamma^{2}\right) \partial_{\gamma} \tilde{\eta}_{c}, \\
v_{1} & =\left(1-\partial_{s}^{2}\right)^{1 / 2}\left(h_{1}+\gamma \tilde{\eta}_{c}\right)=\left(1-\partial_{s}^{2}\right)^{-1 / 2}\left(\left(1-\gamma^{2}+\tilde{\eta}_{c}\right) h_{1}-\gamma \chi\right) .
\end{aligned}
$$

We find that $L h_{1}=2 \gamma \chi$, and with the definitions

$$
z_{0}=\left(\partial_{s} \tilde{\eta}_{c}, S \chi\right), \quad z_{1}=\gamma^{-1}\left(h_{1}, v_{1}\right), \quad w_{0}=\left(\chi,\left(1-\partial_{s}^{2}\right)^{-1 / 2} \chi\right),
$$

we compute that

$$
\mathscr{A} z_{0}=0, \quad \mathscr{A} z_{1}=z_{0}, \quad \mathscr{A}^{*} w_{0}=0 .
$$

Here $z_{0}$ and $z_{1}$ are elements of $X_{a}$, and $w_{0}$ is in $X_{-a}$, which is the dual of $X_{a}$ with respect to the $L^{2}$ pairing.

It is straightforward to show that if $\mathscr{A} z=0$ then the first component of $z$ is a multiple of $\partial_{s} \tilde{\eta}_{c}$ and then that the kernel of $\mathscr{A}$ is one dimensional. To show that zero is an eigenvalue of algebraic multiplicity exactly two for $\mathscr{A}$ on $X_{a}$, it suffices to show that $\left\langle w_{0}, z_{1}\right\rangle \neq 0$. (If the algebraic multiplicity were greater than two, there would exist $z_{2} \in X_{a}$ with $\mathscr{A} z_{2}=z_{1}$, and hence $\left\langle w_{0}, z_{1}\right\rangle=\left\langle\mathscr{A}^{*} w_{0}, z_{2}\right\rangle=0$.) But, using that explicitly $\tilde{\eta}_{c}(s)=\gamma^{2} \Theta(\gamma s)$ from Section 2, so that $\gamma \partial_{\gamma} \tilde{\eta}_{c}=2 \tilde{\eta}_{c}+s \partial_{s} \tilde{\eta}_{c}$, we can compute that

$$
\begin{aligned}
\gamma^{2}\left\langle w_{0}, z_{1}\right\rangle & =\gamma \int_{-\infty}^{\infty} \chi h_{1}+\chi\left(h_{1}+\gamma \tilde{\eta}_{c}\right) \\
& =\int_{-\infty}^{\infty}\left(1-\partial_{s}^{2}\right) \tilde{\eta}_{c}\left(\left(4+\gamma^{2}\right) \tilde{\eta}_{c}+2\left(1-\gamma^{2}\right) s \partial_{s} \tilde{\eta}_{c}\right) d s \\
& =\int_{-\infty}^{\infty}\left(3+2 \gamma^{2}\right) \tilde{\eta}_{c}^{2}+5\left(\partial_{s} \tilde{\eta}_{c}\right)^{2}>0 .
\end{aligned}
$$

This establishes property R2. 
To establish R3 using a perturbation argument, it suffices to show that there exists a positive constant $C_{*}$ such that if $\gamma>0$ is sufficiently small and $a$ has a suitable value depending on $\gamma$, then

$$
\left\|\left(\lambda-\mathscr{A}_{* a}\right)^{-1}\left(\mathscr{A}_{a}-\mathscr{A}_{* a}\right)\right\| \leq \frac{1}{2}
$$

provided only that $|\lambda|>C$ * with $\Re \lambda \geq 0$. For, from (11.11) it follows that the resolvent

$$
\left(\lambda-\mathscr{A}_{a}\right)^{-1}=\left(I-\left(\lambda-\mathscr{A}_{* a}\right)^{-1}\left(\mathscr{A}_{a}-\mathscr{A}_{*}\right)\right)^{-1}\left(\lambda-\mathscr{A}_{* a}\right)^{-1}
$$

is uniformly bounded for $|\lambda|>C_{*}$ with $\Re \lambda>0$. Provided that we show that the resolvent set contains all nonzero $\lambda$ with $\mathfrak{R} \lambda \geq 0$, since the resolvent is analytic in $\lambda$ it follows that the resolvent is uniformly bounded outside of any neighborhood of the origin.

To establish (11.11), we use the representation in (11.6) and (11.7). The norm of the operator of multiplication by $\tilde{\eta}_{c}$ is bounded by $C_{1} \gamma^{2}$ from Theorem 2.1. We claim that if $0<c_{0}<1 \sqrt{3}$, there exists $C_{2}>0$ such that if we take $a=c_{0} \gamma$, then

$$
\sup _{-\infty<\xi<\infty}|\hat{\mathscr{Q}}(i \xi-a)| \leq C_{2} \gamma^{-1}
$$

if $|\lambda|>1$ with $\Re \lambda \geq 0$. Then it follows that $\left\|\mathscr{Q} \tilde{\eta}_{c}\right\| \leq C_{1} C_{2} \gamma$, so (11.11) holds for sufficiently small $\gamma$.

To prove (11.12), we note that $\hat{S}(i \xi-a)$ is uniformly bounded, and let $q_{ \pm}=1 /\left(\lambda-\mathscr{P}_{ \pm}(\mu)\right)$. Then since $\mathscr{P}_{ \pm}(\mu)=\mu \pm \hat{S}(\mu) / c$, we have

$$
\hat{\mathscr{Q}}(\mu)=-\frac{1}{2} \hat{S}(\mu)\left(\begin{array}{cc}
c\left(q_{+}-q_{-}\right) & 0 \\
q_{+}+q_{-} & 0
\end{array}\right) .
$$

Hence we may infer that

$$
\sup _{-\infty<\xi<\infty}|\hat{\mathscr{Q}}(i \xi-a)| \leq \frac{C}{\inf _{\xi}\left|\lambda-\mathscr{P}_{-}(i \xi-a)\right|} .
$$

Here (11.5) was used. From Lemma 6.3 and Taylor's theorem we deduce that if $|\xi|>1$ then $\Re \mathscr{P}_{-}(i \xi-a)<\mathfrak{R}_{-}(i-a)<-a / 2$ for sufficiently small $a>0$. Hence for $|\lambda|>1$ with $\Re \lambda>0$, the infimum above is greater than $a / 2=c_{0} \gamma / 2$, and (11.12) follows. This finishes the proof of R3 in the case B1. 
11.2. Convective linear stability for the case B2

The linear evolution equation for $z=(h, v)$ from (3.1.B3) may be written as $\partial_{\tau} z=\mathscr{A} z=\left(\mathscr{A}_{*}+\mathscr{B}_{*}\right) z$, where

$$
\begin{aligned}
& \mathscr{A}_{*}=\left(\begin{array}{ccc}
\partial_{s} & -\partial_{s} \\
-\left(1-\gamma^{2}\right)\left(1-\partial_{s}^{2}\right)^{-1} \partial_{s} & \partial_{s}
\end{array}\right), \\
& \mathscr{B}_{*}=-\frac{1}{3} \partial_{s}\left(\begin{array}{cc}
\tilde{u}_{c} & \tilde{\eta}_{c} \\
\left(1-\partial_{s}^{2}\right)^{-1} \tilde{u}_{c} & 0
\end{array}\right) .
\end{aligned}
$$

The constant coefficient system $\partial_{\tau} z=\mathscr{A}_{*} z$ admits the conserved quantity

$$
\int_{-\infty}^{\infty} \frac{1}{2}\left(1-\gamma^{2}\right) h^{2}+\frac{1}{2} v^{2}+\frac{1}{2}\left(\partial_{s} v\right)^{2}
$$

which can be used to show that the initial value problem is well posed on the space $X_{0}=L^{2} \times H^{1}$, in the sense that $\mathscr{A}_{*}$ is the generator of a $C_{0}$ semigroup on this space. For this reason we seek to establish properties R1-R3 for $\mathscr{A}$ on the space $X_{a}=L_{a}^{2} \times H_{a}^{1}$, where $H_{a}^{1}$ is the Hilbert space of functions $f$ such that $e^{a s} f$ is in $H^{1}$. The operator $\mathscr{B}_{*}$ is not bounded on this space, due to its first diagonal entry, and $\left(\lambda-\mathscr{A}_{*}\right)^{-1} \mathscr{B}_{*}$ is not compact, so the methods used in case B1 do not apply. To establish R2 and R3, we make direct estimates of Green's function for the resolvent equation $(\lambda-\mathscr{A}) z=\zeta$.

First consider property R1. The function $\tilde{u}_{c}$ is smooth and rapidly decaying, and it is not hard to prove that $\left(1-\frac{1}{3} \tilde{u}_{c}\right) \partial_{s}$ is the generator of a $C_{0}$ semigroup on $L^{2}$, by using characteristics to solve the differential equation $\partial_{\tau} h+\left(1-\frac{1}{3} \tilde{u}_{c}\right) \partial_{s} h=0$ for smooth initial data, and making energy estimates. Now, the operator $\mathscr{A}_{a}=e^{a s} \mathscr{A} e^{-a s}$ is a bounded perturbation of the diagonal operator $\operatorname{diag}\left(\left(1-\frac{1}{3} \tilde{u}_{c}\right) \partial_{s}, \partial_{s}\right)$ in the space $X_{0}$. Hence $\mathscr{A}_{a}$ is the generator of a $C_{0}$ semigroup in $X_{0}$, and R1 follows.

Next, we show that $\lambda=0$ is an eigenvalue of $\mathscr{A}$ on $X_{a}$ with geometric multiplicity one and algebraic multiplicity at least two. Let $z_{0}=\left(\partial_{s} \tilde{\eta}_{c}, \partial_{s} \tilde{u}_{c}\right)$, then $\mathscr{A} z_{0}=0$, and if $\mathscr{A} z=0$ with $z \in X_{a}$, then using the asymptotic theory of ordinary differential equations and Lemma 9.2 one may deduce that $z$ is a constant multiple of $z_{0}$. We may find $z_{1} \in X_{a}$ by observing from (2.1.B3) that $(\eta, u)=\left(\tilde{\eta}_{c}, c \tilde{u}_{c}\right)$ satisfy

$$
-c \partial_{s} \eta+\partial_{s}\left(u+\frac{1}{3} \eta u\right)=0, \quad\left(1-\partial_{s}\right)^{-1} \partial_{s}\left(\eta+\frac{1}{6} u^{2}\right)-c \partial_{s} u=0
$$


Differentiating with respect to $c=\left(1-\gamma^{2}\right)^{-1 / 2}$, we find that $z_{1}=$ $\left(c \partial_{c} \tilde{\eta}_{c}, \partial_{c}\left(c \tilde{u}_{c}\right)\right)$ satisfies $\mathscr{A} z_{1}=z_{0}$ and that $z_{0}$ and $z_{1}$ are in $X_{a}$ provided $0 \leq a<\gamma$.

For further progress, we describe a solution formula for the resolvent equation $(\lambda-\mathscr{A}) z=\zeta$. It suffices to consider $\zeta$ to be smooth and have compact support, since the set of such functions is dense in $X_{a}$. If $\zeta=(f, g)$, we may reduce the resolvent equation to a first-order system for $y=$ $\left(v, \partial_{s} v, \partial_{s}^{2} v, h\right)$ and write it in the form

$$
\partial_{s} y-A(s, \lambda, \gamma) y=\chi=\phi+\partial_{s} \psi
$$

where $A$ is given by (7.2.B2) and

$$
\begin{aligned}
& \phi=\left(0,0, g+\left(1-\gamma^{2}\right)\left(1+\frac{1}{3} \tilde{\eta}_{c}\right) f,-\left(1+\frac{1}{3} \tilde{\eta}_{c}\right) f\right)^{t}, \\
& \psi=\left(0,0,-\partial_{s} g, 0\right)^{t} .
\end{aligned}
$$

The system (11.14) has the solution formula [60, p. 65]

$$
y(s)=\int_{-\infty}^{\infty} K(s, \sigma, \lambda) \chi(\sigma) d \sigma
$$

where the matrix-valued Green's function $K$ may be determined by solving

$$
\begin{aligned}
\partial_{s} K & =A(s, \lambda, \gamma) K, \quad K(\sigma+, \sigma, \lambda)-K(\sigma-, \sigma, \lambda)=I, \\
K(\sigma+, \sigma, \lambda) & =\frac{y^{+}(\sigma, \lambda) z^{-}(\sigma, \lambda)}{D(\lambda)}
\end{aligned}
$$

We have suppressed the dependence of $K$ on $\gamma$. We note that $\partial_{\sigma} K+$ $K A(\sigma, \lambda)=0$, and also

$$
\begin{array}{cc}
K(s, \sigma, \lambda)=\frac{y^{+}(s, \lambda) z^{-}(\sigma, \lambda)}{D(\lambda)} & \text { for } s>\sigma, \\
z^{-}(s, \lambda) K(s, \sigma, \lambda)=0=K(s, \sigma, \lambda) y^{+}(\sigma, \lambda) & \text { for } s<\sigma .
\end{array}
$$


The norm of an integral operator on $L_{a}^{2}$ given by $f \mapsto \int_{-\infty}^{\infty} k(s, \sigma) f(\sigma) d \sigma$ may be bounded by

$$
\begin{aligned}
N_{a}(k)= & \sup _{-\infty<s<\infty} \int_{-\infty}^{\infty}|k(s, \sigma)| e^{a(s-\sigma)} d \sigma \\
& +\sup _{-\infty<\sigma<\infty} \int_{-\infty}^{\infty}|k(s, \sigma)| e^{a(s-\sigma)} d s,
\end{aligned}
$$

using the generalized Young's inequality [66, p. 13]. To bound the resolvent $(\lambda-\mathscr{A})^{-1}$ on $X_{a}$, it is enough to bound the map $e^{a s}(f, g) \mapsto e^{a s}\left(h, v, \partial_{s} v\right)$ from $L^{2} \times H^{1}$ to $\left(L^{2}\right)^{3}$, and since

$$
\int_{-\infty}^{\infty} K(s, \sigma, \lambda) \partial_{\sigma} \psi(\sigma) d \sigma=\psi(s)-\int_{-\infty}^{\infty} \partial_{\sigma} K(s, \sigma, \lambda) \psi(\sigma) d \sigma
$$

clearly the norm of the resolvent on $X_{a}$ may be bounded by $\left\|(\lambda-\mathscr{A})^{-1}\right\| \leq$ $5 \Upsilon_{a}(K(\lambda))$, where

$$
\Upsilon_{a}(K(\lambda))=\max _{i=1,2,4, j=3,4} N_{a}\left(K_{i j}(\lambda)\right)+\max _{i=1,2,4} N_{a}\left(\partial_{\sigma} K_{i 3}(\lambda)\right) .
$$

In (11.19) the $(s, \sigma)$ arguments of $K$ have been suppressed.

From the expressions in (11.16) and (11.17) we see that the Green's function $K(s, \sigma, \lambda)$ is meromorphic in $\lambda$ on the domain $\Omega^{\gamma}$ of the Evans function and that zeros of the Evans function $D(\lambda)$ induce poles in the resolvent formula. (This is exactly why such zeros are called resonance poles if they are not eigenvalues in $X_{0}$.) Our Corollary 5.2 implies that if $\gamma>0$ is sufficiently small and $\lambda=\frac{1}{2} \gamma^{3} \Lambda \in \frac{1}{2} \gamma^{3} \mathscr{S}^{\gamma}$, then there is only one such pole possible, at $\lambda=0$.

Regarding the Green's function $K$, we establish the following estimates.

LEMMA 11.2. Suppose $a=c_{0} \gamma$, where $0<c_{0}<1 / \sqrt{3}$, and $\gamma>0$ is suffciently small. Then the following hold.

(a) There are positive constants $C$ and $\tilde{C}$ such that whenever $\Re \lambda>0$ and $|\lambda|>\tilde{C}$

$$
\Upsilon_{a}(K(\lambda))<C
$$

(b) If $\lambda \neq 0$ and $\mathfrak{R} \lambda>-b$, where $0<b<-\mathscr{P}_{-}(-a)$, then

$$
\Upsilon_{a}(K(\lambda))<\infty .
$$

(c) There are positive constants $C$ and $\delta$ such that whenever $0<|\lambda|<\delta$,

$$
|D(\lambda)|^{2} \Upsilon_{a}(K(\lambda))<C .
$$


Part (b) of this lemma implies that $\lambda$ is in the resolvent set of $\mathscr{A}$ on $X_{a}$, if $\lambda \neq 0$ with $\mathfrak{R} \lambda>\mathscr{P}_{-}(-a)$. The resolvent is analytic on its resolvent set, so property R3 follows from parts (a) and (b) of the lemma.

We next use part (c) to prove property R2. Since $\lambda=0$ is a zero of order two for $D(\lambda)$, it follows that $\lambda^{4}(\lambda-\mathscr{A})^{-1}$ is analytic at $\lambda=0$. It is evident from (11.16) that $\lambda^{3} K(s, \sigma, \lambda) \rightarrow 0$ as $\lambda \rightarrow 0$, uniformly for $(s, \sigma)$ in compact sets. We infer that the first two terms of the Taylor expansion of $\lambda^{4}(\lambda-\mathscr{A})^{-1}$ vanish (e.g., for $\chi$ of compact support, $\lambda^{4}(\lambda-\mathscr{A})^{-1} \chi \rightarrow 0$ weakly, hence strongly, hence the first term vanishes).

So the resolvent of $\mathscr{A}$ has the expansion

$$
(\lambda-\mathscr{A})^{-1}=\frac{D_{0}}{\lambda^{2}}+\frac{P_{0}}{\lambda}+R_{0}(\lambda),
$$

where $R_{0}$ is analytic. The operator $P_{0}$ is the spectral projection on the generalized null space of $A$. The meaning of R2 is that $P_{0}$ has rank two. We can find an explicit representation of $P_{0}$. Let $K_{*}(s, \sigma, \lambda)=D(\lambda) K(s, \sigma, \lambda)$; then clearly $K_{*}$ is analytic in a neighborhood of $\lambda=0$. From (11.16) we see that $K_{*}$ is continuous at $s=\sigma$ when $\lambda=0$, and in fact for all $s$ and $\sigma$ we have

$$
\begin{aligned}
K_{*}(s, \sigma, 0) & =y^{+}(s, 0) z^{-}(\sigma, 0), \\
\partial_{\lambda} K_{*}(s, \sigma, 0) & =y_{\lambda}^{+}(s, 0) z^{-}(\sigma, 0)+y^{+}(s, 0) z_{\lambda}^{-}(\sigma, 0) .
\end{aligned}
$$

Here $y_{\lambda}^{+}=\partial_{\lambda} y^{+}, z_{\lambda}^{-}=\partial_{\lambda} z^{-}$. Expansion of $K_{*}$ and $D$ then reveals that there are constants $\beta_{0}, \beta_{1}$ such that

$$
\begin{aligned}
P_{0} \chi(s)= & \int_{-\infty}^{\infty}\left(\beta_{0} \partial_{\lambda} K_{*}+\beta_{1} K_{*}\right) \chi d \sigma \\
= & y_{\lambda}^{+}(s, 0) \int_{-\infty}^{\infty} \beta_{0} z^{-}(\sigma, 0) \chi(\sigma) d \sigma \\
& +y^{+}(s, 0) \int_{-\infty}^{\infty}\left(\beta_{0} z_{\lambda}^{-}+\beta_{1} z^{-}\right)(\sigma, 0) \chi(\sigma) d \sigma .
\end{aligned}
$$

Clearly $P_{0}$ has rank two at most. But we have shown above that the generalized kernel of $\mathscr{A}$ is at least two dimensional. Therefore $P_{0}$ has exactly rank two, proving $\mathrm{R} 2$.

It remains to prove Lemma 11.2. To obtain the estimates we seek regarding the Green's function $K$, we make considerable use of results concerning exponential dichotomies for systems of ordinary differential equations, as described by Coppel [66]. 
Let us first prove part (a). As in Section 10, we change variables to diagonalize $A^{\infty}$, letting $V=V(\lambda)$ be the associated matrix of right eigenvectors and $W=V^{-1}$ the matrix of left eigenvectors. Define

$$
K^{a}(s, \sigma, \lambda)=e^{a(s-\sigma)} W K(s, \sigma, \lambda) V
$$

then $K^{a}$ is Green's function for the system

$$
\partial_{s} v=B_{a}(s) v+\tilde{\chi}
$$

where we may write

$$
B_{a}(s)=W A(s, \lambda, \gamma) V+a I=\tilde{B}_{a}(s)+\tilde{F}(s) .
$$

Here, as in (10.15) and (10.16),

$$
\begin{aligned}
\tilde{B}_{a}(s)= & \left(\begin{array}{cccc}
\mu_{1}+a & 0 & 0 & 0 \\
0 & \mu_{2}+a & 0 & 0 \\
0 & 0 & \mu_{3}+a & 0 \\
0 & 0 & 0 & \mu_{4}+a
\end{array}\right) \\
& +\frac{1}{6} \tilde{\eta}_{c}(s) \lambda\left(\begin{array}{rrrc}
0 & 0 & 0 & 0 \\
0 & 1 & -1 & 0 \\
0 & -1 & 1 & 0 \\
0 & 0 & 0 & 0
\end{array}\right),
\end{aligned}
$$

and we have the estimate

$$
|\tilde{F}(s)| \leq C \gamma^{2} e^{-|\gamma s|}
$$

for $\lambda \in S_{4}$ if $\delta$ is sufficiently small.

Now note that

$$
\begin{aligned}
e^{a(s-\sigma)} K_{i j}(s, \sigma, \lambda) & =\left(e_{i}^{t} V\right) K^{a}(s, \sigma)\left(W e_{j}\right), \\
e^{a(s-\sigma)} \partial_{\sigma} K_{i j}(s, \sigma, \lambda) & =-\left(e_{i}^{t} V\right) K^{a}(s, \sigma)\left(B_{0}(s) W e_{j}\right) .
\end{aligned}
$$

Using the asymptotic estimates in (10.12) for $\mu_{j}$ and the representation of the eigenvectors in (7.4) and (7.5), one may deduce without difficulty that

$$
\max _{i=1,2,4, j=3,4}\left|e_{i}^{t} V\right|\left|W e_{j}\right| \leq C, \quad \max _{i=1,2,4}\left|e_{i}^{t} V \| B_{0}(s) W e_{3}\right| \leq C
$$

uniformly for $\lambda \in S_{4}$ and $-\infty<s<\infty$, if $\delta$ is sufficiently small. 
To prove part (a), it suffices to show that if $\gamma$ is sufficiently small, then $N_{0}\left(\left|K^{a}(\lambda)\right|\right)$ is uniformly bounded for $|\lambda|$ large with $\mathfrak{R} \lambda>0$. We derive this bound from the analogous bound on the Green's function $\tilde{K}$ of the simpler system

$$
\partial_{s} v=\tilde{B}_{a}(s) v+\tilde{\chi},
$$

by exploiting Coppel's analysis of the "roughness" of exponential dichotomies [66]. To explain, let $\Phi(s)$ be the matrix satisfying

$$
\partial_{s} \Phi=B_{a}(s) \Phi, \quad \Phi(0)=I .
$$

Define $P=D(\lambda)^{-1} W y^{+}(0, \lambda) z^{-}(0, \lambda) V$. The matrix $P=P^{2}$ and is the projection on the space of initial conditions at $s=0$ for solutions of $\partial_{s} v=B_{a} v$ that are $O\left(\exp \left(\mu_{1}+a\right) s\right)$ as $s \rightarrow+\infty$. Also, $I-P$ is the corresponding projection for solutions that are $O\left(\exp \left(\mu_{*}+a\right) s\right)$ as $s \rightarrow-\infty$. The Green's function $K^{a}$ is given by the formula

$$
K^{a}(s, \sigma)= \begin{cases}\Phi(s) P \Phi(\sigma)^{-1} & \text { for } s>\sigma \\ -\Phi(s)(I-P) \Phi(\sigma)^{-1} & \text { for } s<\sigma .\end{cases}
$$

The dependence on $(\lambda, \gamma)$ has been suppressed here, but we claim that, for sufficiently small $\gamma>0$ and $a=c_{0} \gamma$ as described above, there are positive constants $C_{0}$ and $\alpha$ independent of $\lambda$ such that

$$
\begin{aligned}
\left|\Phi(s) P \Phi(\sigma)^{-1}\right| \leq C_{0} e^{-\alpha(s-\sigma)} & \text { for } s>\sigma, \\
\left|\Phi(s)(I-P) \Phi(\sigma)^{-1}\right| \leq C_{0} e^{-\alpha(\sigma-s)} & \text { for } s<\sigma,
\end{aligned}
$$

for any $\lambda$ with $|\lambda|$ sufficiently large and $\Re \lambda>0$. Part (a) of the lemma follows directly. The inequalities (11.22) assert that system $\partial_{s} v=B_{a} v$ admits an exponential dichotomy with projection $P$, having structure constants $C_{0}$ and $\alpha$ independent of $\lambda$.

Because of the estimate (11.20) and Coppel's results on the persistence of exponential dichotomies (see Proposition 1 on p. 34 and the end of chapter 2 in [66]), the claim in (11.22) is a direct consequence of corresponding estimates for the simpler system (11.21). To get such estimates, let $\tilde{\Phi}(s)$ satisfy

$$
\partial_{s} \tilde{\Phi}=\tilde{B}_{a}(s) \tilde{\Phi}, \quad \tilde{\Phi}(0)=I,
$$


and define $\tilde{P}=e_{1} e_{1}^{t}$. The Green's function $\tilde{K}$ for (11.21) is

$$
\tilde{K}(s, \sigma)= \begin{cases}\tilde{\Phi}(s) \tilde{P} \tilde{\Phi}(\sigma)^{-1} & \text { for } s>\sigma \\ -\tilde{\Phi}(s)(I-\tilde{P}) \tilde{\Phi}(\sigma)^{-1} & \text { for } s<\sigma .\end{cases}
$$

Clearly $\tilde{K}$ has the same block diagonal structure as $\tilde{B}_{a}(s)$, and in fact,

$$
\begin{aligned}
& \tilde{K}_{11}(s, \sigma)= \begin{cases}\exp \left(\mu_{1}+a\right)(s-\sigma), & s>\sigma, \\
0, & s<\sigma,\end{cases} \\
& \tilde{K}_{44}(s, \sigma)= \begin{cases}0, & s>\sigma, \\
-\exp \left(\mu_{4}+a\right)(s-\sigma), & s<\sigma .\end{cases}
\end{aligned}
$$

The $2 \times 2$ matrix

$$
G(s, \sigma)=\left(\begin{array}{ll}
\tilde{K}_{22} & \tilde{K}_{23} \\
\tilde{K}_{32} & \tilde{K}_{33}
\end{array}\right)
$$

satisfies $G(s, \sigma)=0$ for $s>\sigma$ and, for $s<\sigma$, satisfies

$$
\partial_{s} G=\left(\begin{array}{cc}
\mu_{2}+a & 0 \\
0 & \mu_{3}+a
\end{array}\right) G+\frac{1}{6} \tilde{\eta}_{c} \lambda\left(\begin{array}{rr}
1 & -1 \\
-1 & 1
\end{array}\right) G, \quad G(\sigma-0, \sigma)=I
$$

Since $\mathfrak{R} \mu_{j}>0$ for $\mathfrak{i} \lambda>0$ if $j>1$, using the energy method as at the end of Section 10 we find that

$$
\operatorname{tr} \bar{G}^{t} G(s, \sigma) \leq 2 e^{a(s-\sigma)} \quad \text { for } s<\sigma .
$$

We infer that, since $\Re \mu_{1}+a<-\frac{1}{2}$ for $|\lambda|$ large with $\Re \lambda>0$,

$$
\begin{aligned}
\left|\tilde{\Phi}(s) \tilde{P} \tilde{\Phi}(\sigma)^{-1}\right| \leq C_{0} e^{-(s-\sigma) / 2} & \text { for } s>\sigma, \\
\left|\tilde{\Phi}(s)(I-\tilde{P}) \tilde{\Phi}(\sigma)^{-1}\right| \leq C_{0} e^{-a(\sigma-s)} & \text { for } s<\sigma,
\end{aligned}
$$

where $C_{0}$ is independent of $\lambda$ and $\gamma$. As we have mentioned, from this, the choice $a=c_{0} \gamma$, the estimate in (11.20) and Proposition 1 of [66], follow the inequalities in (11.22). This finishes the proof of part (a).

It remains to prove parts (b) and (c) of Lemma 11.2. We do this by using results in [66] to establish the existence of an exponential dichotomy for

$$
\partial_{s} y=(A(s, \lambda)+a I) y
$$


with appropriate bounds on the structure constants that correspond to $C_{0}$ and $\alpha$ in (11.22).

Suppose $a=c_{0} \gamma$ where $0<c_{0}<1 / \sqrt{3}$ and $0<b<-\mathscr{P}_{-}(-a)$ and $\gamma$ is sufficiently small so that $D(\lambda, \gamma) \neq 0$ whenever $\lambda \neq 0$ and $\mathfrak{R} \lambda>-b$. Fix any compact set $\Omega_{0}$ in the half-plane $\mathfrak{i} \lambda>-b$. Supposing $\Re \lambda>-b$, we have that $\mathfrak{R} \mu_{1}+a<0<\mathfrak{R} \mu_{j}+a, j>1$. Hence, the constant coefficient system

$$
\partial_{s} y=\left(A^{\infty}(\lambda)+a I\right) y
$$

has an exponential dichotomy with projection $P_{0}=v_{1} w_{1}$, uniformly for $\lambda \in \Omega_{0}$. That is, if $\Phi(s)$ is the fundamental matrix of this system satisfying $\Phi(0)=I$, then positive constants $C_{0}$ and $\alpha$ exist, such that estimates (11.22) hold, uniformly for $\lambda \in \Omega_{0}$.

Using Coppel's Proposition 1 and the decay properties of the deviator $A(s, \lambda, \gamma)-A^{\infty}(\lambda, \gamma)$, which were established in Section 7, it follows that there exist exponential dichotomies for (11.23) on semi-infinite intervals $\left(-\infty,-s_{0}\right]$ and $\left[s_{0}, \infty\right)$ (with $s_{0}$ independent of $\left.\lambda \in \Omega_{0}\right)$ and thence on $(-\infty, 0]$ and $[0, \infty)\left[66\right.$, p. 13]. The respective projections, $Q^{\prime \prime}$ and $Q^{\prime}$, are such that $Q^{\prime}$ has the same nullspace as $P_{0}=v_{1} w_{1}$, and $I-Q^{\prime \prime}$ has the same nullspace as $I-P$. In fact, $Q^{\prime}$ is a matrix of rank one, given by

$$
Q^{\prime}=\frac{y^{+}(0) w_{1} \Phi\left(s_{0}\right)}{w_{1} \Phi\left(s_{0}\right) y^{+}(0)} .
$$

(Note $w_{1} \Phi\left(s_{0}\right) y^{+}(0)=w_{1} y^{+}\left(s_{0}\right) \neq 0$.) The structure constants for these exponential dichotomies may be fixed independent of $\lambda \in \Omega_{0}$. That is, if $\Phi(s)$ is the fundamental matrix of (11.23) satisfying $\Phi(0)=I$, then positive constants $C_{1}, \alpha_{1}$ exist, such that for all $\lambda \in \Omega_{0}$ we have

$$
\begin{aligned}
\left|\Phi(s) Q^{\prime} \Phi(\sigma)^{-1}\right| \leq C_{1} e^{-\alpha_{1}(s-\sigma)} & \text { for } s>\sigma \geq 0, \\
\left|\Phi(s)\left(I-Q^{\prime}\right) \Phi(\sigma)^{-1}\right| \leq C_{1} e^{-\alpha_{1}(\sigma-s)} & \text { for } 0 \leq s<\sigma, \\
\left|\Phi(s) Q^{\prime \prime} \Phi(\sigma)^{-1}\right| \leq C_{1} e^{-\alpha_{1}(s-\sigma)} & \text { for } 0 \geq s>\sigma, \\
\left|\Phi(s)\left(I-Q^{\prime \prime}\right) \Phi(\sigma)^{-1}\right| \leq C_{1} e^{-\alpha_{1}(\sigma-s)} & \text { for } s<\sigma \leq 0 .
\end{aligned}
$$

The range of $Q^{\prime}$ is the space of initial conditions at $s=0$ for solutions of (11.23), which remain bounded on $[0, \infty)$; this space is spanned by $y^{+}(0)$. The range of $I-Q^{\prime \prime}$ is the space of initial conditions at $s=0$ for solutions that remain bounded on $(-\infty, 0]$; this is the space orthogonal to $z^{-}(0)$. Provided $D(\lambda)=z^{-}(0) y^{+}(0) \neq 0$, these two spaces do not intersect, and the projection

$$
P=\frac{y^{+}(0) z^{-}(0)}{D(\lambda)}
$$


has the same range as $Q^{\prime}$ and the same nullspace as $Q^{\prime \prime}$. By the discussion in $[66$, p. 16], this means that (11.23) admits exponential dichotomies on $(-\infty, 0]$ and $[0, \infty)$, with the same projection in each case, namely $P$. This change of projections in each case requires a change in the structure constants, which is analyzed by Coppel [66, p. 17]. The result is that we may assert that for $\lambda \in \Omega_{0}$ we have the estimates

$$
\begin{aligned}
\left|\Phi(s) P \Phi(\sigma)^{-1}\right| \leq C_{2}^{\prime}(\lambda) e^{-\alpha_{1}(s-\sigma)} & \text { for } s>\sigma \geq 0, \\
\left|\Phi(s)(I-P) \Phi(\sigma)^{-1}\right| \leq C_{2}^{\prime}(\lambda) e^{-\alpha_{1}(\sigma-s)} & \text { for } 0 \leq s<\sigma, \\
\left|\Phi(s) P \Phi(\sigma)^{-1}\right| \leq C_{2}^{\prime \prime}(\lambda) e^{-\alpha_{1}(s-\sigma)} & \text { for } 0 \geq s>\sigma, \\
\left|\Phi(s)(I-P) \Phi(\sigma)^{-1}\right| \leq C_{2}^{\prime \prime}(\lambda) e^{-\alpha_{1}(\sigma-s)} & \text { for } s<\sigma \leq 0,
\end{aligned}
$$

where

$$
C_{2}^{\prime}(\lambda)=\left(1+C_{1}\left|P-Q^{\prime}\right|\right) C_{1} \leq C_{3}|D(\lambda)|^{-1}
$$

for some constant $C_{3}$ independent of $\lambda \in \Omega_{0}$. There is a corresponding expression and estimate for $C_{2}^{\prime \prime}$.

Since the projection is the same for each half-line, there is an exponential dichotomy for (11.23) on the whole line [66, p. 19]. We estimate the structure constants by writing, for $s>0>\sigma$, for example,

$$
\Phi(s) P \Phi(\sigma)^{-1}=\left(\Phi(s) P \Phi(0)^{-1}\right)\left(\Phi(0) \Phi(\sigma)^{-1}\right) .
$$

Then it follows that for $\lambda \in \Omega_{0}$ we have

$$
\begin{aligned}
\left|\Phi(s) P \Phi(\sigma)^{-1}\right| \leq C_{4}(\lambda) e^{-\alpha_{1}(s-\sigma)} & \text { for } s>\sigma, \\
\left|\Phi(s)(I-P) \Phi(\sigma)^{-1}\right| \leq C_{4}(\lambda) e^{-\alpha_{1}(\sigma-s)} & \text { for } s<\sigma,
\end{aligned}
$$

where $C_{4}(\lambda)=C_{3}^{2}|D(\lambda)|^{-2}$.

The point of these estimates is that the Green's function $K$ of (11.14) satisfies

$$
e^{a(s-\sigma)} K(s, \sigma)= \begin{cases}\Phi(s) P \Phi(\sigma)^{-1} & \text { for } s>\sigma \\ -\Phi(s)(I-P) \Phi(\sigma)^{-1} & \text { for } s<\sigma\end{cases}
$$

From these estimates it is easy to verify parts (b) and (c) and Lemma 11.2. 


\section{Acknowledgments}

This work was partially supported by the National Science Foundation. The authors thank P. Smereka and H. Segur for discussions involving this work and an anonymous referee for comments that inspired improvements in the paper. M.I.W. is pleased to acknowledge the hospitality of the Mittag-Leffler Institute during October 1994, and the Program in Applied and Computational Mathematics at Princeton University during 1995-96, where part of this work was completed.

\section{References}

1. M. J. Boussineso, Théorie de l'intumescence liquide, applelée onde solitaire ou de translation, se propageant dans un canal rectangulaire, C. R. Acad. Sci. Paris 72:755-759 (1871).

2. M. J. BoussinesQ, Théorie générale des mouvements qui sont propagés dans un canal rectangulaire horizontal, C. R. Acad. Sci. Paris 73:256-260 (1871).

3. M. J. BoussinesQ, Théorie des ondes et des remous qui se propagent le long d'un canal rectangulaire horizontal, en communiquant au liquide contenu dans ce canal des vitesses sensiblement pareilles de la surface au fond, J. Math. Pures Appl. 17:55-108 (1872).

4. M. J. BoussinesQ, Essai sur la théorie des eaux courantes, Mémoires présentés par divers savants á l'Académie des Sciences Inst. France (séries 2) 23:1-680 (1877).

5. J. Scott Russell, Report on waves, Report, 14th Meeting of the British Association for the Advancement of Science, pp. 311-390+57 plates, John Murray, London, 1844.

6. G. B. AIRY, Tides and waves, Encycl. Metropolitana 5 (1845).

7. G. StOKes, On the theory of oscillatory waves, (1847). In Mathematical and Physical Papers, Johnson Reprint Corp., New York. (See Section 5.)

8. G. B. Whitham, Linear and Nonlinear Waves, Wiley, New York, 1974.

9. P. Deift, C. TomeI, and E. Trubowitz, Inverse scattering and the Boussinesq equation, Comm. Pure Appl. Math. 35:567-628 (1982).

10. V. E. ZaKharov, On the stochastization of one dimensional chains of nonlinear oscillators, Soviet Phys. JETP 38:108-110 (1974).

11. S. K. Turitsyn, Blow-up in the Boussinesq equation, Phys. Rev. E 47:R796-799 (1993).

12. T. B. Benjamin, J. L. Bona, and J. J. MAHONY, Model equations for long waves in nonlinear dispersive media, Philos. Trans. Roy. Soc. London Ser. A 272:47-78 (1972).

13. P. Rosenau, Dynamics of dense lattices, Phys. Rev. B 36:5868-5876 (1987).

14. D. H. Peregrine, Calculations of the development of an undular bore, J. Fluid Mech. 25:321-330 (1966)

15. M. SCHONBEK, Existence of solutions for the Boussinesq system of equations, J. Differential Equations 42:325-352 (1981).

16. C. Aмick, Regularity and uniqueness of solutions to the Boussinesq system of equations, J. Differential Equations 54:231-247 (1984).

17. R. L. Pego and M. I. Weinstein, Asymptotic stability of solitary waves, Comm. Math. Phys. 164:305-349 (1994).

18. M. Reed and B. Simon, Methods of Modern Mathematical Physics IV: Analysis of Operators, Academic Press, New York, 1978. 
19. P. D. LaX and R. S. Phillips, Scattering Theory, Academic Press, New York, 1967.

20. J. D. Crawford and P. D. Hislop, Application of the method of spectral deformation to the Vlasov Poisson system, Ann. Physics 189:265-317 (1989).

21. J. D. CRAwFord and P. D. Hislop, Application of the method of spectral deformation to the Vlasov Poisson system. II. Mathematical results, J. Math. Phys. 30:2819-2837 (1989).

22. S. H. Strogatz, R. E. Mirollo, and P. C. Matthews, Coupled nonlinear oscillators below the synchronization threshold: Relaxation by generalized Landau damping, Phys. Rev. Lett. 68(18):2730-2733 (1992).

23. J. Alexander, R. Gardner, and C. K. R. T. Jones, A topological invariant arising in the stability analysis of traveling waves, J. Reine Angew. Math. 410:167-212 (1990).

24. J. W. Evans, Nerve axon equations. IV. The stable and unstable impulse, Indiana Univ. Math. J. 24:1169-1190 (1975).

25. L. Gearhart, Spectral theory for contraction semigroups on Hilbert space, Trans. Amer. Math. Soc. 236:385-394 (1978).

26. J. PrÜss, On the spectrum of $C_{0}$-semigroups, Trans. Amer. Math. Soc. 284:847-857 (1984).

27. F. HuAng, Characteristic condition for exponential stability of linear dynamical systems in Hilbert spaces, Ann. Differential Equations 1:43-56 (1985).

28. J. R. Miller, Asymptotic stability of the regularized long wave equation, Ph.D. Thesis, University of Michigan, 1994.

29. J. R. Miller and M. I. Weinstein, Asymptotic stability of solitary waves for the regularized long wave equation, Comm. Pure Appl. Math. 49:399-441 (1996).

30. J. DoDD, Convective stability of shock profile solutions of a modified KdV-Burgers equation, Ph.D. Thesis, University of Maryland, 1996.

31. A. SOFfer and M. I. Weinstein, Multichannel nonlinear scattering theory for nonintegrable equations. In Integrable Systems and Applications (M. Balabane, P. Lochak, and C. Sulem, Eds.), Lecture Notes in Physics, Vol. 342, pp. 312-327, Springer, New York, 1988.

32. A. Soffer and M. I. WeinsteIn, Multichannel nonlinear scattering theory for nonintegrable equations, Comm. Math. Phys. 133:119-146 (1990).

33. A. SofFER and M. I. Weinstein, Multichannel nonlinear scattering theory for nonintegrable equations. II. The case of anisotropic potentials and data, J. Differential Equations 98:376-390 (1992).

34. N. J. BALMForth, Solitary waves and homoclinic orbits, Ann. Rev. Fluid Mech. 27:335-373 (1995).

35. H.-C. Chang, Wave evolution on a falling film, Ann. Rev. Fluid Mech. 26:103-136 (1994).

36. H.-C. Chang, E. A. Demekhin, and D. I. Kopelevich, Local stability theory of solitary pulses in an active medium, Phys. D, 97:353-375 (1996).

37. T. Ogawa and H. SuZuKI, On the spectra of pulses in a nearly integrable system, SIAM J. Appl. 57:485-500 (1997).

38. T. B. Benjamin, The stability of solitary waves, Proc. Roy. Soc. London Ser. A 328:153-183 (1972).

39. J. L. Bona, P. E. Souganidis, and W. A. Strauss, Stability and instability of solitary waves, Proc. Roy. Soc. London Ser. A 411:395-412 (1987).

40. M. Grillakis, J. Shatah, and W. Strauss, Stability theory of solitary waves in the presence of symmetry, I, J. Funct. Anal. 74:160-197 (1988).

41. D. B. Henry, J. F. Perez, and W. F. Wreszinski, Stability theory for solitary wave solutions of scalar field equations, Commun. Math. Phys. 85:351-361 (1982). 
42. E. W. LAedke and K. H. SpatscheK, Stability theorem for KdV type equations, J. Plasma Phys. 32:263-272 (1984).

43. M. I. WeinsteIn, Modulational stability of ground states of nonlinear Schrödinger equations, SIAM J. Math. Anal. 16:472-491 (1985).

44. M. I. Weinstein, On the solitary traveling wave of the generalized Korteweg-de Vries equation. In Nonlinear Systems of Partial Differential Equations in Applied Mathematics (B. Nicolaenko, D. Holm, and J. Hyman, Eds.), Lectures in Appl. Math., Vol. 23, Part 2, Amer. Math. Soc., Providence, 1986.

45. M. I. WEINSTEIN, Lyapunov stability of ground states of nonlinear dispersive evolution equations, Comm. Pure Appl. Math. 39:51-68 (1986).

46. M. I. Weinstein, Existence and dynamical stability theory of solitary wave solutions of equations governing long wave propagation, Comm. Partial Differential Equations 12:1133-1173 (1987).

47. T. Cazenave and P.-L. Lions, Orbital stability of standing waves for some nonlinear Schrödinger equations, Commun. Math. Phys. 85:549-561 (1982).

48. V. I. ARNOL'D, Conditions for nonlinear stability of stationary curvilinear flows of an ideal fluid, Dokl. Mat. Nauk. 162:773-777 (1965).

49. D. D. Holm, J. E. Marsden, T. Ratiu, and A. Weinstein, Nonlinear stability of fluid and plasma equilibria, Phys. Rep. 123:1-116 (1985).

50. M. D. Kruskal and C. R. Oberman, On the stability of plasma in static equilibrium, Phys. Fluids 1:275-280 (1958).

51. P. Smereka, A remark on the solitary wave stability for a Boussinesq equation. In Nonlinear Dispersive Wave Systems (L. Debnath, Ed.), pp. 255-263, World Scientific, Singapore, 1992.

52. R. L. Pego, P. Smereka, and M. I. Weinstein, Oscillatory instability of solitary waves in a continuum model of lattice vibrations, Nonlinearity 8:921-941 (1995).

53. J. L. BonA and R. SACHS, The existence of internal solitary waves in a two-fluid system near the KdV limit, Geophys. Astrophys. Fluid Dynamics 48:25-51 (1989).

54. D. J. KorteweG and G. DE VRIES, On the change of form of long waves advancing in a rectangular channel, and on a new type of long stationary waves, Philos. Mag. (5) 39:422-443 (1895).

55. J. L. Bona, On the stability of solitary waves, Proc. Roy. Soc. London Ser. A 344:363-374 (1975).

56. J. W. Miles, The Korteweg-de Vries equation: A historical essay, J. Fluid Mech. 106:131-147 (1981).

57. E. A. Coddington and N. Levinson, Theory of Ordinary Differential Equations, McGrawHill, New York, 1955.

58. J. G. Berryman, Stability of solitary waves in shallow water, Phys. Fluids 19:771-777 (1976).

59. A. JeFFrey and T. KAKUTANI, Weakly nonlinear dispersive waves: A discussion centered about the Korteweg-de Vries equation, SIAM Rev. 14:582-643 (1972).

60. R. L. Pego and M. I. Weinstein, Eigenvalues, and instabilities of solitary waves, Philos. Trans. Roy. Soc. London Ser. A 340:47-94 (1992).

61. C. K. R. T. Jones, Stability of the traveling wave solution to the FitzHugh-Hagumo equation, Trans. Amer. Math. Soc. 286:431-469 (1984).

62. W. A. Coppel, Stability and Asymptotic Behavior of Differential Equations, D.C. Heath, Boston, 1965. 
63. R. L. Pego and M. I. Weinstein, Evans' function, Melnikov's integral and solitary wave instabilities. In Differential Equations with Applications to Mathematical Physics (W. F. Ames, J. V. Herod, and E. M. Harrell, Eds.), pp. 273-286, Academic Press, Orlando, 1993.

64. I. C. Gohberg and M. G. KREIN, Introduction to the Theory of Linear Nonselfadjoint Operators, Transl. Math. Monog., Vol. 18, Amer. Math. Soc., Providence, 1969.

65. R. L. Pego, Compactness in $L^{2}$ and the Fourier transform, Proc. Amer. Math. Soc. 95:252-254 (1985).

66. G. B. Folland, Introduction to Partial Differential Equations, Princeton Univ. Press, 1976.

67. W. A. Coppel, Dichotomies in Stability Theory, Lecture Notes in Mathematics, Vol. 629, Springer-Verlag, New York, 1978.

University of Maryland, College Park
University of Michigan, Ann Arbor

(Received March 8, 1995) 